$$
\text { for }
$$

\title{
Palladium-Catalyzed Decarbonylative Alkylation of Acyl Fluorides
}

\author{
Liyan $\mathrm{Fu},{ }^{\dagger}$ Qiang Chen, ${ }^{\dagger}$ Zhenhua Wang, ${ }^{\dagger}$ and Yasushi Nishihara ${ }^{\ddagger}, *$ \\ † Graduate School of Natural Science and Technology, Okayama University, \\ 3-1-1 Tsushimanaka, Kita-ku, Okayama 700-8530, Japan \\ ${ }^{\dagger}$ Research Institute for Interdisciplinary Science, Okayama University, \\ 3-1-1 Tsushimanaka, Kita-ku, Okayama 700-8530, Japan \\ Phone: +81-86-251-7855 \\ Fax: $+81-86-251-7855$ \\ Email: ynishiha@okayama-u.ac.jp
}

1. General

2. Optimization of Reaction Conditions

3. Experimental Procedures and Spectroscopic Data for the Products

$S 10-S 24$

4. Copies of ${ }^{1} H,{ }^{13} C\left\{{ }^{1} H\right\}$, and ${ }^{19} F\left\{{ }^{1} H\right\}$ NMR Charts for the Products

S25-S57

5. References

$S 58$ 


\section{General}

\section{Instrumentation}

Unless otherwise noted, all the reactions were carried out under an Ar atmosphere using standard Schlenk techniques. Solvents were employed as eluents for all other routine operation, and dehydrated solvents were purchased from commercial suppliers and employed without any further purification. Glassware was dried in an oven $\left(130^{\circ} \mathrm{C}\right)$ and heated under reduced pressure before use. For thin layer chromatography (TLC) analyses throughout this work, Merck precoated TLC plates (silica gel $60 \mathrm{GF}_{254}, 0.25 \mathrm{~mm}$ ) were used. Silica gel column chromatography was carried out using Silica gel $60 \mathrm{~N}$ (spherical, neutral, 40-100 $\mu \mathrm{m}$ ) from Kanto Chemicals Co., Ltd. NMR spectra $\left({ }^{1} \mathrm{H},{ }^{13} \mathrm{C}\left\{{ }^{1} \mathrm{H}\right\}\right.$, and $\left.{ }^{19} \mathrm{~F}\left\{{ }^{1} \mathrm{H}\right\}\right)$ were recorded on Varian INOVA-600 (600 MHz) or Mercury-400 (400 MHz) spectrometers. Chemical shifts $(\delta)$ are in parts per million relative to $\mathrm{CDCl}_{3}$ at 7.26 ppm for ${ }^{1} \mathrm{H}$ and at $77.16 \mathrm{ppm}$ for ${ }^{13} \mathrm{C}\left\{{ }^{1} \mathrm{H}\right\}$, respectively. The ${ }^{19} \mathrm{~F}\left\{{ }^{1} \mathrm{H}\right\}$ NMR spectra were measured by using $\mathrm{CCl}_{3} \mathrm{~F}(\delta=0.00 \mathrm{ppm})$ as an external standard. The $\mathrm{GC}$ yields were determined by $\mathrm{GC}$ analysis of the crude mixture, using $n$-dodecane as an internal standard. GC analyses were performed on a Shimadzu GC-14A equipped with a flame ionization detector using Shimadzu Capillary Column (CBP1-M25-025) and Shimadzu C-R6A-Chromatopac integrator. Infrared spectra were recorded on a Shimadzu IR Prestige-21 spectrophotometer. Elemental analyses were carried out with a Perkin-Elmer $2400 \mathrm{CHN}$ elemental analyzer at Okayama University.

\section{Chemicals}

Unless otherwise noted, materials obtained from commercial suppliers were used without further purification. Bis(2,4-pentanedionato)palladium(II), 1,2-bis(diphenylphosphino)ethane, benzoyl fluoride (1a) (purity > 98\%), and allylbenzene (purity > 98\%) were purchased from Tokyo Chemical Industry Co., Ltd.. 9Borabicyclo[3.3.1]nonane dimer was purchased from Sigma-Aldrich Co. Potassium fluoride (purity $>95 \%$ ) was obtained from Nacalai Tesque. Dimethyl sulfoxide (super dehydrated) was purchased from Kanto Chemical Co. Acyl fluorides 1b-1t were prepared according to the literatures ${ }^{1}$ and showed the identical spectra reported. 


\section{Optimization of Reaction Conditions}

Table S1. Screening of Catalysts and Ligands ${ }^{a}$

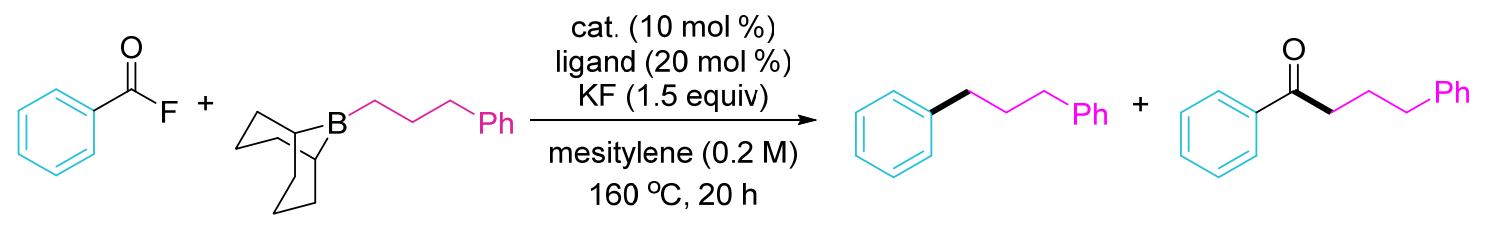

$1 \mathrm{a}$

2a ( 1.5 equiv)

3aa

4

\begin{tabular}{|c|c|c|c|c|}
\hline \multirow{2}{*}{ entry } & \multirow{2}{*}{ cat. } & \multirow{2}{*}{ ligand } & \multicolumn{2}{|c|}{ yield $(\%)^{b}$} \\
\hline & & & Заa & 4 \\
\hline 1 & $\mathrm{Ni}(\mathrm{cod})_{2}$ & DCYPE & 0 & 1 \\
\hline 2 & $\mathrm{Pd}(\mathrm{OAc})_{2}$ & DCYPE & 38 & 2 \\
\hline 3 & $\operatorname{Pd}(\mathrm{dba})_{2}$ & DCYPE & 42 & 3 \\
\hline 4 & $\operatorname{Pd}(\mathrm{acac})_{2}$ & DCYPE & 44 & 1 \\
\hline 5 & $\operatorname{Pd}(\mathrm{tfa})_{2}$ & DCYPE & 39 & 4 \\
\hline $6^{c}$ & $\mathrm{Pd}_{2}(\mathrm{dba})_{3}$ & DCYPE & 33 & 3 \\
\hline 7 & $\mathrm{PdCl}_{2}$ & DCYPE & 28 & 2 \\
\hline 8 & $\operatorname{Pd}(\mathrm{acac})_{2}$ & DPPM & 4 & 14 \\
\hline 9 & $\operatorname{Pd}(\mathrm{acac})_{2}$ & DPPE & 44 & 4 \\
\hline 10 & $\operatorname{Pd}(\text { acac })_{2}$ & DPPP & 17 & 24 \\
\hline 11 & $\operatorname{Pd}(\mathrm{acac})_{2}$ & DPPF & 10 & 12 \\
\hline 12 & $\mathrm{Pd}(\mathrm{acac})_{2}$ & XantPhos & 19 & 0 \\
\hline 13 & $\operatorname{Pd}(\text { acac })_{2}$ & $r a c$-BINAP & 20 & 24 \\
\hline 14 & $\operatorname{Pd}(\text { acac })_{2}$ & $\mathrm{PPh}_{3}{ }^{d}$ & 10 & 41 \\
\hline
\end{tabular}

${ }^{a}$ Reactions were carried out with 1a $(0.2 \mathrm{mmol}, 1.0$ equiv), 2a $(0.3 \mathrm{~mL}, 1.0 \mathrm{M}$ in mesitylene, $0.3 \mathrm{mmol}, 1.5$ equiv) and cat. (0.02 mmol, $10 \mathrm{~mol} \%)$, [P] (0.04 mmol, $20 \mathrm{~mol} \%), \mathrm{KF}(0.3 \mathrm{mmol}, 1.5$ equiv) in mesitylene $(0.7 \mathrm{~mL})$ at $160{ }^{\circ} \mathrm{C}$ for $20 \mathrm{~h} .{ }^{b} \mathrm{GC}$ yields, using $n$-dodecane as the internal standard. ${ }^{c} 5 \mathrm{~mol} \% . \quad{ }^{d} 40 \mathrm{~mol} \%$.

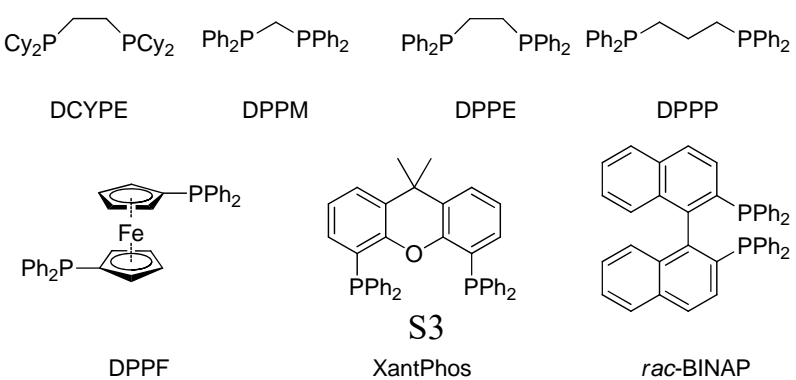


Table S2. Screening of Base ${ }^{a}$

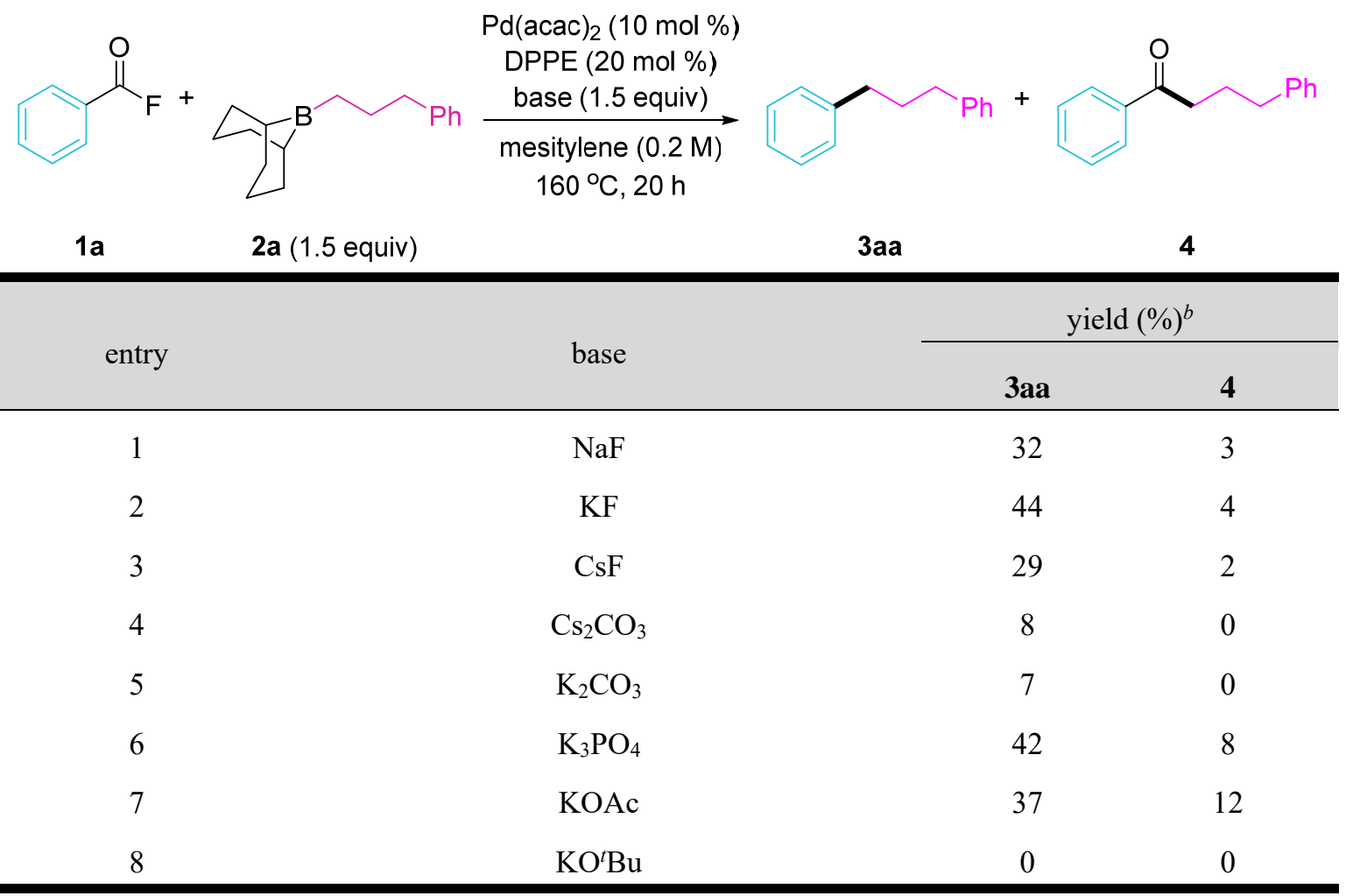

${ }^{a}$ Reactions were carried out with 1a $(0.2 \mathrm{mmol}, 1.0$ equiv), 2a $(0.3 \mathrm{~mL}, 1.0 \mathrm{M}$ in mesitylene, $0.3 \mathrm{mmol}, 1.5$ equiv) and $\operatorname{Pd}(\mathrm{acac})_{2}(0.02 \mathrm{mmol}, 10 \mathrm{~mol} \%)$, DPPE (0.04 mmol, $\left.20 \mathrm{~mol} \%\right)$, base (0.3 mmol, 1.5 equiv) in mesitylene $(0.7 \mathrm{~mL})$ at $160{ }^{\circ} \mathrm{C}$ for $20 \mathrm{~h} . \quad{ }^{b} \mathrm{GC}$ yields, using $n$-dodecane as the internal standard. 
Table S3. Screening of Amounts of Ligand and Base ${ }^{a}$

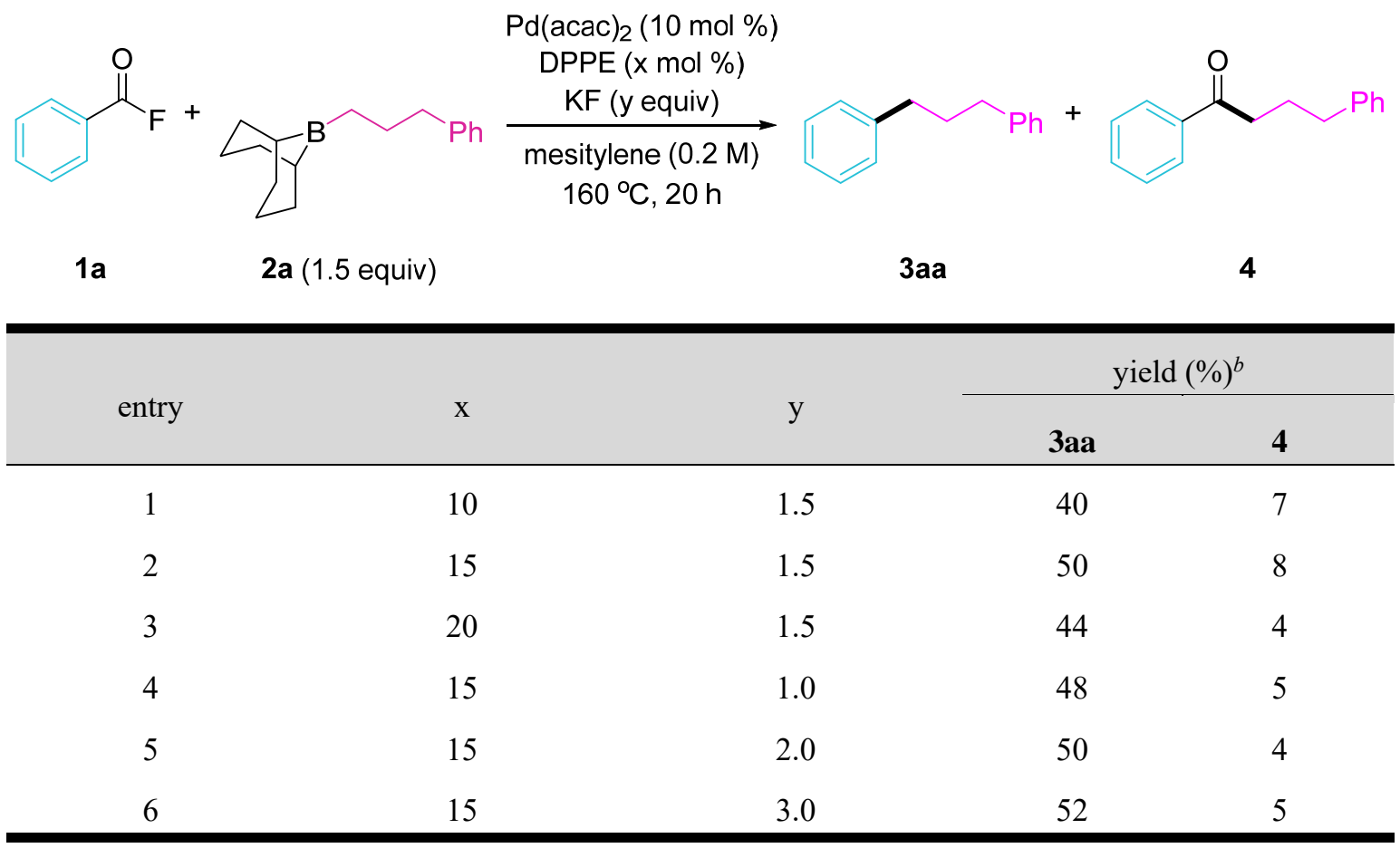

${ }^{a}$ Reactions were carried out with 1a $(0.2 \mathrm{mmol}, 1.0$ equiv), 2a $(0.3 \mathrm{~mL}, 1.0 \mathrm{M}$ in mesitylene, $0.3 \mathrm{mmol}, 1.5$ equiv) and $\mathrm{Pd}(\mathrm{acac})_{2}(0.02 \mathrm{mmol}, 10 \mathrm{~mol} \%)$, DPPE (x mol \%), KF (y equiv) in mesitylene $(0.7 \mathrm{~mL})$ at $160{ }^{\circ} \mathrm{C}$ for $20 \mathrm{~h} . \quad{ }^{b} \mathrm{GC}$ yields, using $n$-dodecane as the internal standard. 
Table S4. Screening of Temperature ${ }^{a}$

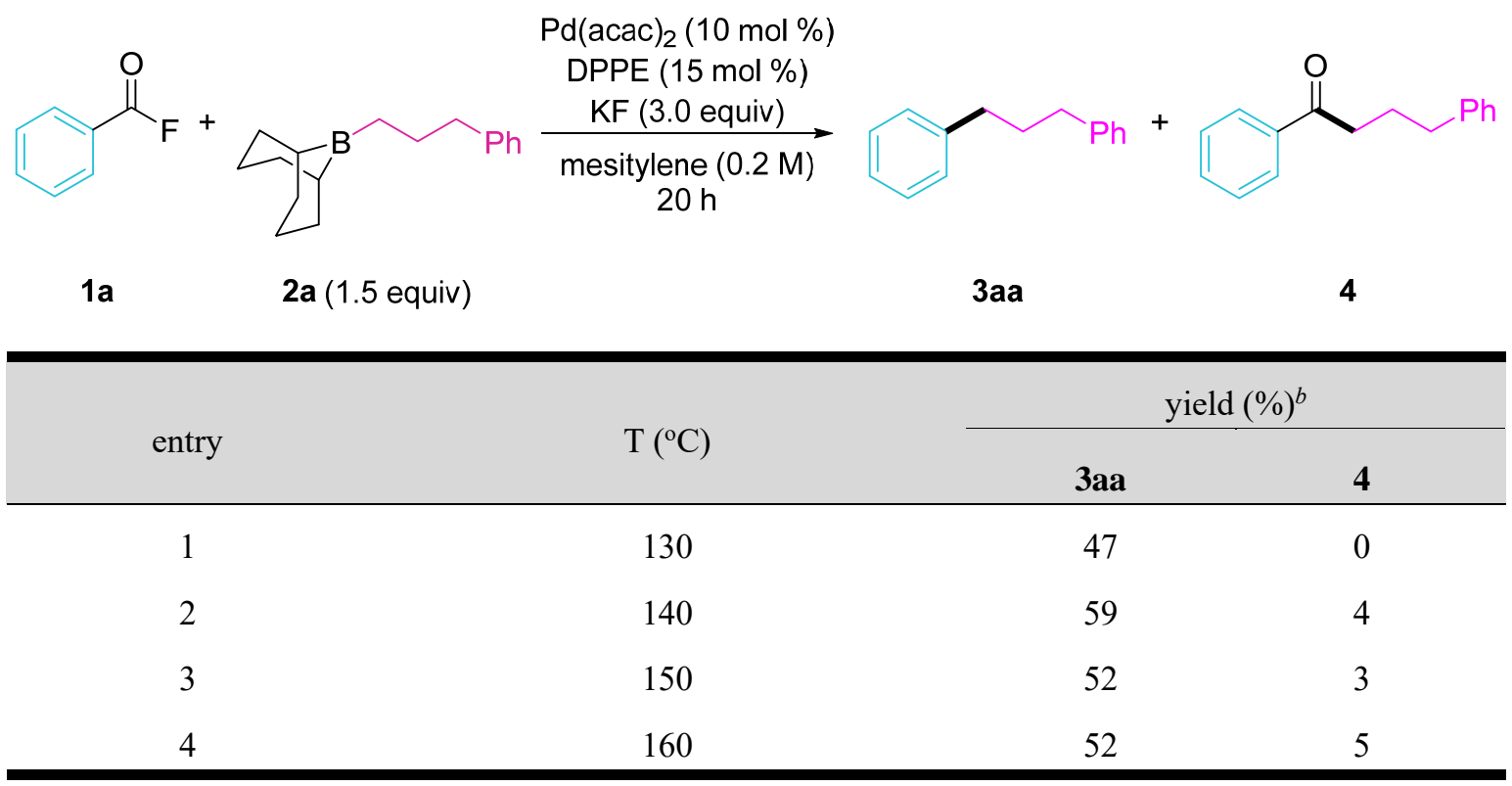

${ }^{a}$ Reactions were carried out with 1a $(0.2 \mathrm{mmol}, 1.0$ equiv), $2 \mathrm{a}(0.3 \mathrm{~mL}, 1.0 \mathrm{M}$ in mesitylene, $0.3 \mathrm{mmol}, 1.5$ equiv) and $\mathrm{Pd}(\mathrm{acac})_{2}(0.02 \mathrm{mmol}, 10 \mathrm{~mol} \%)$, DPPE (0.03 mmol, $\left.15 \mathrm{~mol} \%\right), \mathrm{KF}(0.6 \mathrm{mmol}, 3.0$ equiv) in mesitylene $(0.7 \mathrm{~mL})$ for $20 \mathrm{~h} . \quad{ }^{b} \mathrm{GC}$ yields, using $n$-dodecane as the internal standard. 
Table S5. Screening of Solvent ${ }^{a}$

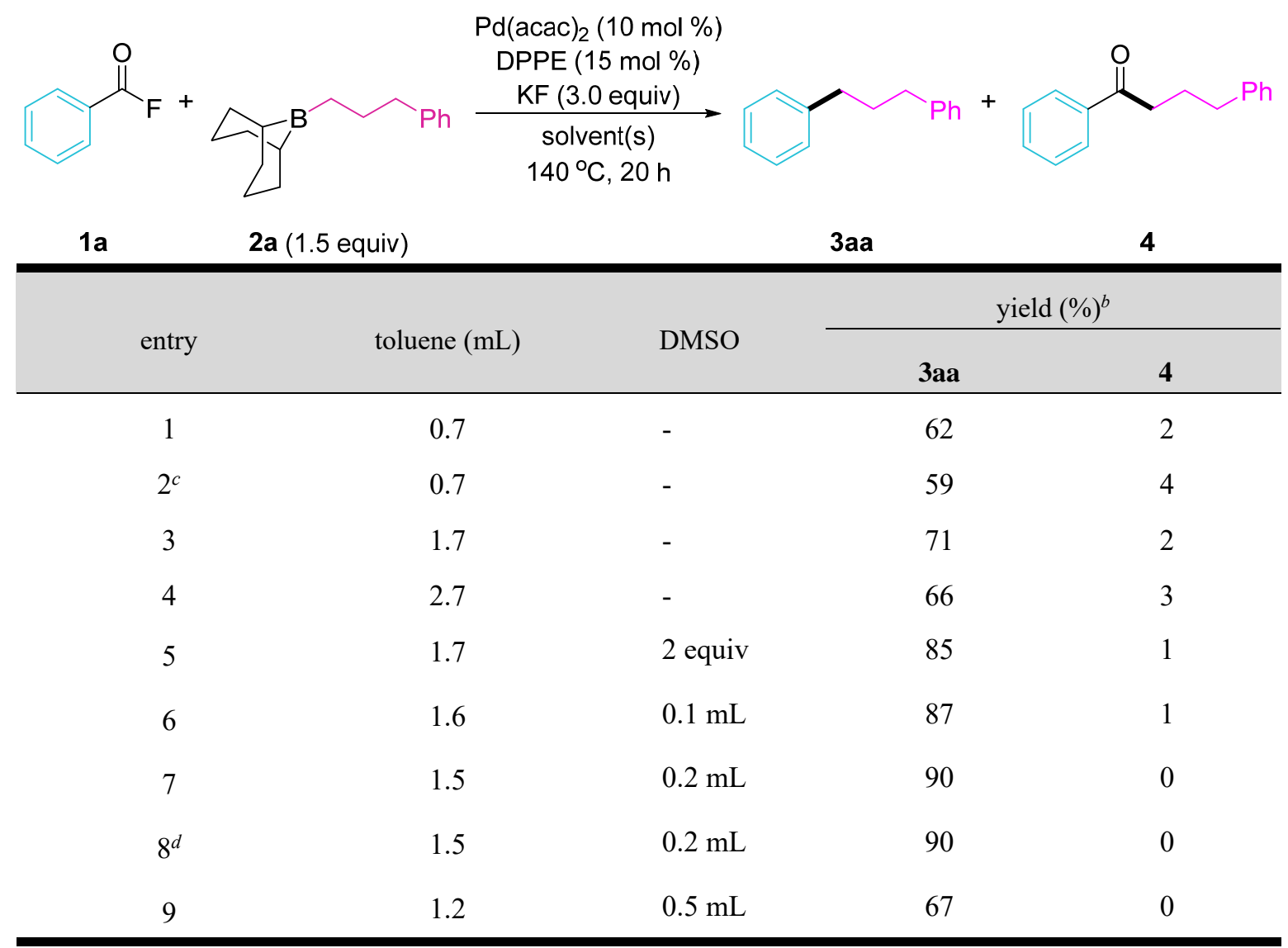

${ }^{a}$ Reactions were carried out with $1 \mathbf{a}(0.2 \mathrm{mmol}, 1.0$ equiv), $2 \mathbf{a}(0.3 \mathrm{~mL}, 1.0 \mathrm{M}$ in toluene, $0.3 \mathrm{mmol}, 1.5$ equiv) and Pd(acac) $)_{2}(0.02 \mathrm{mmol}, 10 \mathrm{~mol} \%)$, DPPE (0.03 mmol, $\left.15 \mathrm{~mol} \%\right)$, KF (0.6 mmol, 3.0 equiv) in solvent at $140{ }^{\circ} \mathrm{C}$ for $20 \mathrm{~h} . \quad{ }^{b} \mathrm{GC}$ yields, using $n$-dodecane as the internal standard. $\quad{ }^{c}$ Mesitylene instead of toluene. $\quad{ }^{d} \mathrm{KF}$ ( $0.4 \mathrm{mmol}, 1.5$ equiv). 
Table S6. Optimization of Reaction Time ${ }^{a}$

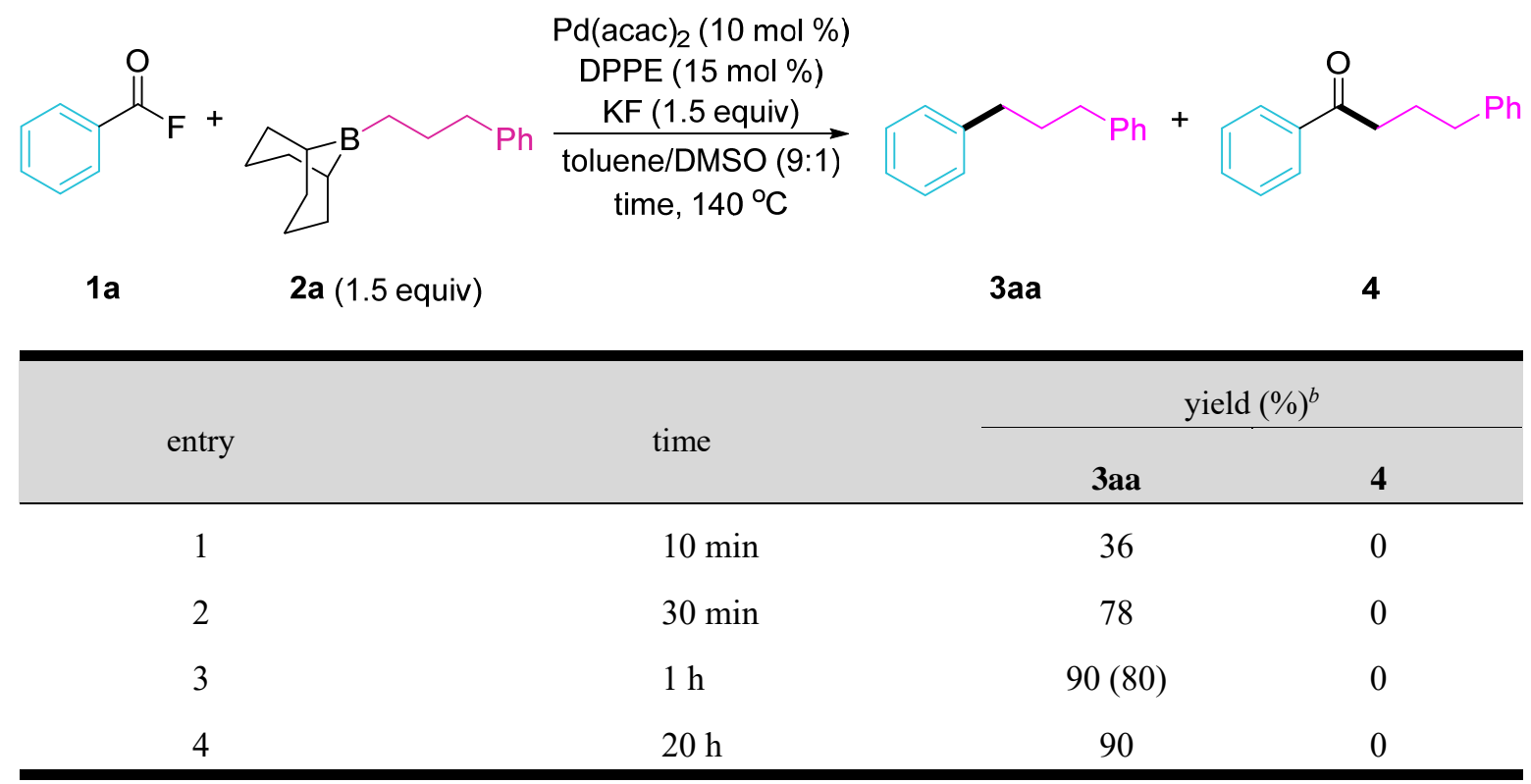

${ }^{a}$ Reactions were carried out with $1 \mathbf{a}(0.2 \mathrm{mmol}, 1.0$ equiv), $2 \mathbf{a}(0.3 \mathrm{~mL}, 1.0 \mathrm{M}$ in toluene, $0.3 \mathrm{mmol}, 1.5$ equiv) and Pd(acac) 2 (0.02 mmol, $10 \mathrm{~mol} \%)$, DPPE (0.03 mmol, $15 \mathrm{~mol} \%)$, KF (0.3 mmol, 1.5 equiv) in toluene (1.5 $\mathrm{mL})$, DMSO $(0.2 \mathrm{~mL})$ at $140{ }^{\circ} \mathrm{C} . \quad{ }^{b} \mathrm{GC}$ yields, using $n$-dodecane as the internal standard. An isolated yield is given in parentheses. 
Table S7. Control Experiments ${ }^{a}$

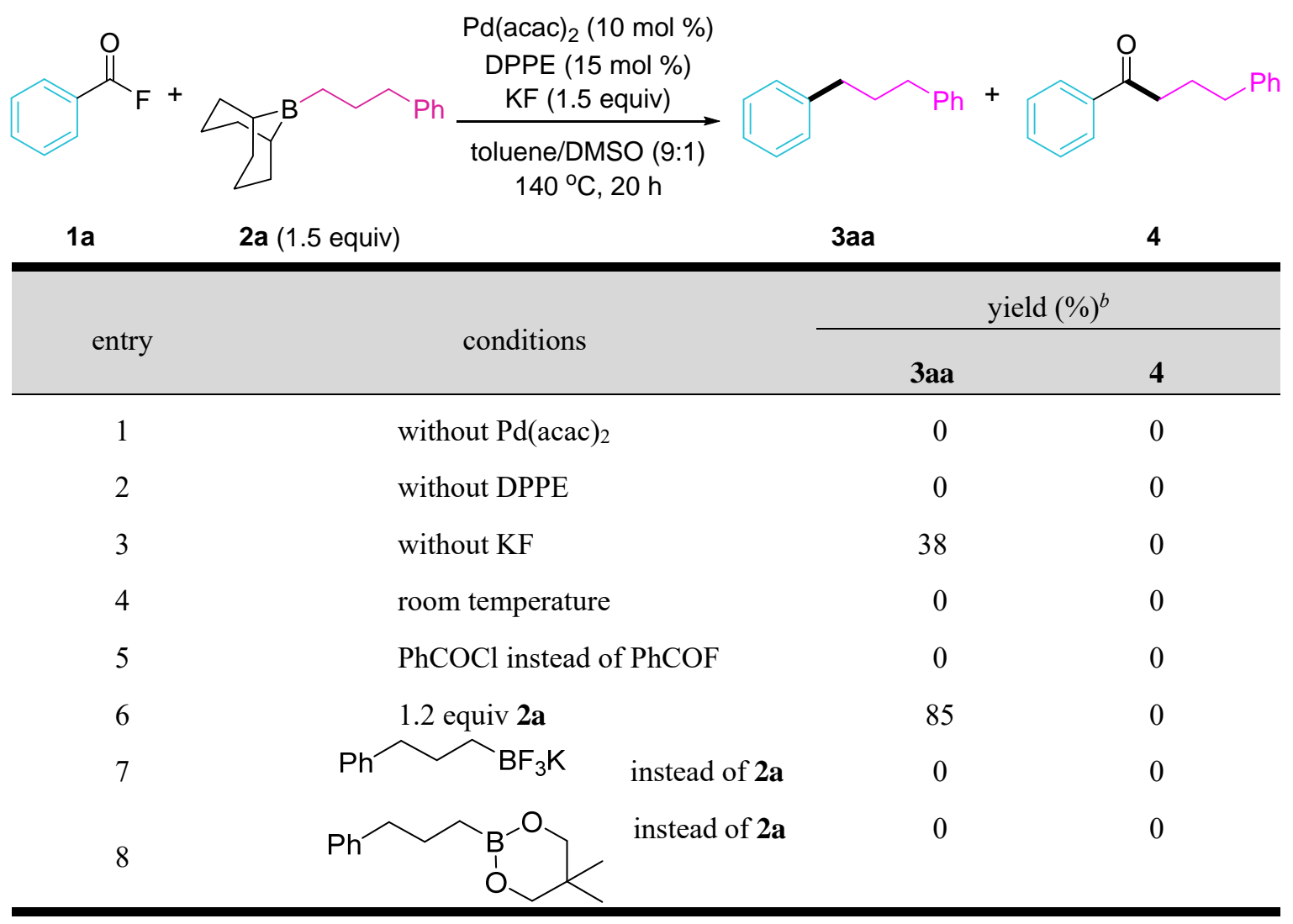

${ }^{a}$ Reactions were carried out with $1 \mathbf{a}(0.2 \mathrm{mmol}, 1.0$ equiv), $2 \mathbf{a}(0.3 \mathrm{~mL}, 1.0 \mathrm{M}$ in toluene, $0.3 \mathrm{mmol}, 1.5$ equiv) and Pd(acac) 2 (0.02 mmol, $10 \mathrm{~mol} \%)$, DPPE (0.03 mmol, $15 \mathrm{~mol} \%), \mathrm{KF}(0.3 \mathrm{mmol}, 1.5$ equiv) in toluene $(1.5 \mathrm{~mL})$, DMSO $(0.2 \mathrm{~mL})$ at $140{ }^{\circ} \mathrm{C}$ for $20 \mathrm{~h} .{ }^{b} \mathrm{GC}$ yields, using $n$-dodecane as the internal standard. 


\section{Experimental Procedures and Spectroscopic Data for the Products}

3.1 General Procedure for Pd-catalyzed Decarbonylative Alkylation of Acyl Fluorides 1 with Alkylboranes 2.

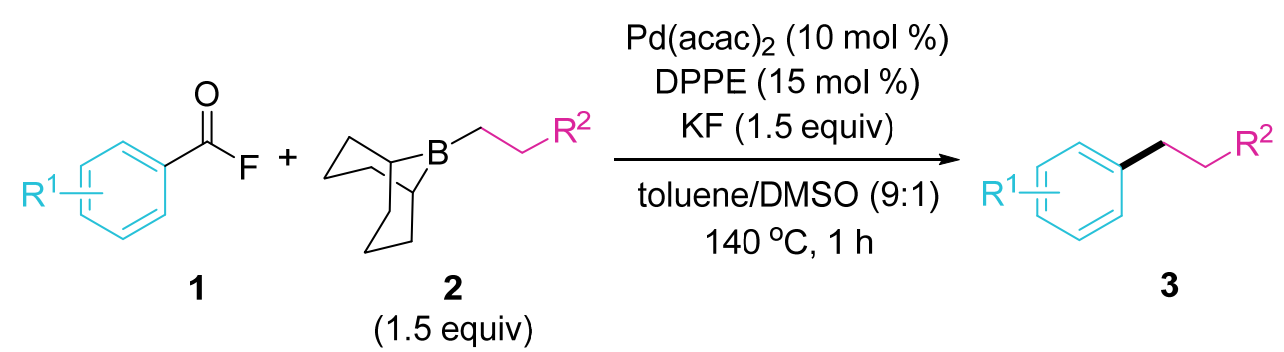

An oven-dried Schlenk tube $(25 \mathrm{~mL})$ containing a magnetic stirring bar was charged with $\operatorname{Pd}(\mathrm{acac})_{2}(6.1 \mathrm{mg}$, $0.02 \mathrm{mmol}, 10 \mathrm{~mol} \%$ ), DPPE (12.0 mg, $0.03 \mathrm{mmol}, 15 \mathrm{~mol} \%$ ), KF (17.4 mg, $0.3 \mathrm{mmol}, 1.5$ equiv), toluene (1.5 $\mathrm{mL})$, and DMSO $(0.2 \mathrm{~mL})$ under argon and the reaction mixture was stirred for $30 \mathrm{sec}$ at room temperature. After the solution of alkyl 9-BBN $2(0.3 \mathrm{~mL} / 1.0 \mathrm{M}, 1.5$ equiv), derived from the reaction of 9-BBN-dimer (1.0 equiv) and the alkene (2.0 equiv) in dry toluene at $80^{\circ} \mathrm{C}$ (oil bath temp) for $3 \mathrm{~h}$, was added to the Schlenk tube, and sequentially acyl fluoride $1\left(0.2 \mathrm{mmol}, 1.0\right.$ equiv) was added. The reaction mixture was heated at $140{ }^{\circ} \mathrm{C}$ with stirring for $1 \mathrm{~h}$. After the mixture was cooled to room temperature, quenched with saturated $\mathrm{NH}_{4} \mathrm{Cl}$, and extracted with $\mathrm{Et}_{2} \mathrm{O}$. The combined organic extracts were dried over anhydrous $\mathrm{MgSO}_{4}$, and filtered and evaporated under vacuum to obtain the crude product which was purified by column chromatography (EtOAc/hexane) on silica gel to afford the desired products 3.

\subsection{Spectroscopic Data for the Products.}

\section{1,3-Diphenylpropane (3aa) $)^{2}$}

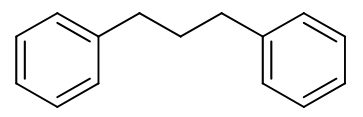

3aa

Colorless oil. $\quad R_{\mathrm{f}}=0.44$ (hexane). Isolated yield is $80 \%(31.3 \mathrm{mg}) . \quad{ }^{1} \mathrm{H} \mathrm{NMR}\left(400 \mathrm{MHz}, \mathrm{CDCl}_{3}\right): \delta 1.96-2.04$ (m, 2H), 2.69 (t, $J=7.8 \mathrm{~Hz}, 4 \mathrm{H}), 7.20-7.23(\mathrm{~m}, 6 \mathrm{H}), 7.30-7.34(\mathrm{~m}, 4 \mathrm{H}) ;{ }^{13} \mathrm{C}\left\{{ }^{1} \mathrm{H}\right\} \mathrm{NMR}\left(101 \mathrm{MHz}, \mathrm{CDCl}_{3}\right): \delta$ $33.1,35.6,125.9,128.4,128.6,142.4$. 
1-Methyl-2-(3-phenylpropyl)benzene (3ba) ${ }^{2}$<smiles>Cc1ccccc1CCCc1ccccc1</smiles>

3ba

Colorless oil. $\quad R_{\mathrm{f}}=0.40$ (hexane). Isolated yield is $93 \%(39.1 \mathrm{mg}) . \quad{ }^{1} \mathrm{H} \mathrm{NMR}\left(400 \mathrm{MHz}, \mathrm{CDCl}_{3}\right): \delta 1.88-1.96$ (m, 2H), 2.28 (s, 3H), 2.65 (t, $J=7.8 \mathrm{~Hz}, 2 \mathrm{H}), 2.71(\mathrm{t}, J=7.8 \mathrm{~Hz}, 2 \mathrm{H}), 7.10-7.14(\mathrm{~m}, 4 \mathrm{H}), 7.18-7.22(\mathrm{~m}, 3 \mathrm{H})$, 7.28-7.32 (m, 2H); ${ }^{13} \mathrm{C}\left\{{ }^{1} \mathrm{H}\right\}$ NMR (101 MHz, $\left.\mathrm{CDCl}_{3}\right): \delta$ 19.4, 31.9, 33.0, 36.0, 125.9, 126.0, 126.0, 128.4, 128.5, $128.9,130.3,136.0,140.6,142.4$.

\section{1-Methyl-3-(3-phenylpropyl)benzene (3ca)}<smiles>Cc1cccc(CCCc2ccccc2)c1</smiles>

3ca

Colorless oil. $\quad R_{\mathrm{f}}=0.36$ (hexane). Isolated yield is $67 \%(28.2 \mathrm{mg}) . \quad{ }^{1} \mathrm{H} \mathrm{NMR}\left(400 \mathrm{MHz}, \mathrm{CDCl}_{3}\right): \delta 1.96-2.03$ (m, 2H), $2.37(\mathrm{~s}, 3 \mathrm{H}), 2.64-2.72(\mathrm{~m}, 4 \mathrm{H}), 7.02-7.05(\mathrm{~m}, 3 \mathrm{H}), 7.20-7.24(\mathrm{~m}, 4 \mathrm{H}), 7.31-7.34(\mathrm{~m}, 2 \mathrm{H}) ;{ }^{13} \mathrm{C}\left\{{ }^{1} \mathrm{H}\right\}$ NMR (101 MHz, $\left.\mathrm{CDCl}_{3}\right): \delta 21.6,33.1,35.5,35.7,125.6,125.8,126.6,128.3,128.4,128.6,129.4,137.9,142.4$, 142.5 .

\section{1-Methyl-4-(3-phenylpropyl)benzene (3da) ${ }^{4}$}<smiles>Cc1ccc(CCCc2ccccc2)cc1</smiles>

3da

Colorless oil. $\quad R_{\mathrm{f}}=0.42$ (hexane). Isolated yield is $59 \%(24.7 \mathrm{mg}) . \quad{ }^{1} \mathrm{H} \mathrm{NMR}\left(400 \mathrm{MHz}, \mathrm{CDCl}_{3}\right): \delta 1.91-1.99$ (m, 2H), 2.33 (s, 3H), 2.61-2.68 (m, 4H), 7.07-7.12 (m, 4H), 7.17-7.21 (m, 3H), 7.27-7.31 (m, 2H); ${ }^{13} \mathrm{C}\left\{{ }^{1} \mathrm{H}\right\}$ NMR (151 MHz, $\left.\mathrm{CDCl}_{3}\right): \delta 21.2,33.2,35.1,35.6,125.8,128.4,128.5,128.6,129.1,135.3,139.3,142.5$. 


\section{1,3,5-Trimethyl-2-(3-phenylpropyl)benzene (3ea) ${ }^{5}$}<smiles>Cc1cc(C)c(CCCc2ccccc2)c(C)c1</smiles>

3ea

Colorless oil. $\quad R_{\mathrm{f}}=0.32$ (hexane). Isolated yield is $87 \%(41.3 \mathrm{mg}) . \quad{ }^{1} \mathrm{H}$ NMR $\left(400 \mathrm{MHz}, \mathrm{CDCl}_{3}\right): \delta 1.75-1.83$ (m, 2H), 2.24 (s, 6H), 2.25 (s, 3H), 2.59-2.63 (m, 2H), 2.76 (t, $J=7.6 \mathrm{~Hz}, 2 \mathrm{H}), 6.83$ (s, 2H), 7.19-7.24 (m, 3H), 7.29-7.33 (m, 2H); ${ }^{13} \mathrm{C}\left\{{ }^{1} \mathrm{H}\right\}$ NMR (101 MHz, $\left.\mathrm{CDCl}_{3}\right): \delta 19.8,20.9,29.1,30.8,36.5,125.9,128.4,128.5,129.0$, $135.0,136.0,136.3,142.4$.

\section{1-(tert-Butyl)-4-(3-phenylpropyl)benzene (3fa) ${ }^{6}$}<smiles>CC(C)(C)c1ccc(CCCc2ccccc2)cc1</smiles>

$3 \mathbf{f a}$

Colorless oil. $\quad R_{\mathrm{f}}=0.50$ (hexane). Isolated yield is $61 \%(30.6 \mathrm{mg}) .{ }^{1} \mathrm{H} \mathrm{NMR}\left(400 \mathrm{MHz}, \mathrm{CDCl}_{3}\right): \delta 1.32$ (s, 9H), 1.93-2.01 (m, 2H), 2.62-2.69 (m, 4H), $7.13(\mathrm{~d}, J=8.0 \mathrm{~Hz}, 2 \mathrm{H}), 7.17-7.21(\mathrm{~m}, 3 \mathrm{H}), 7.27-7.33(\mathrm{~m}, 4 \mathrm{H})$; ${ }^{13} \mathrm{C}\left\{{ }^{1} \mathrm{H}\right\}$ NMR $\left(101 \mathrm{MHz}, \mathrm{CDCl}_{3}\right): \delta 31.6,33.0,34.5,35.0,35.7,125.3,125.8,128.2,128.4,128.6,139.3,142.5$, 148.6 .

4-(3-Phenylpropyl)-1,1'-biphenyl (3ga) ${ }^{2}$<smiles>c1ccc(CCCc2ccc(-c3ccccc3)cc2)cc1</smiles>

Colorless oil. $\quad R_{\mathrm{f}}=0.30$ (hexane). Isolated yield is $88 \%(47.9 \mathrm{mg}) . \quad{ }^{1} \mathrm{H}$ NMR (400 MHz, $\left.\mathrm{CDCl}_{3}\right): \delta 1.98-2.05$ (m, 2H), 2.68-2.73 (m, 4H), 7.19-7.23 (m, 3H), 7.29-7.36 (m, 5H), 7.44 (t, $J=7.6 \mathrm{~Hz}, 2 \mathrm{H}), 7.53$ (d, $J=7.6 \mathrm{~Hz}$, 2H), 7.59-7.61 (m, 2H); ${ }^{13} \mathrm{C}\left\{{ }^{1} \mathrm{H}\right\}$ NMR (101 MHz, $\left.\mathrm{CDCl}_{3}\right): \delta 33.1,35.2,35.6,125.9,127.1(2 \mathrm{C}), 127.2,128.5$, $128.6,128.9,129.0,138.8,141.2,141.6,142.4$. 


\section{1-Methoxy-4-(3-phenylpropyl)benzene (3ha) ${ }^{2}$}<smiles>COc1ccc(CCCc2ccccc2)cc1</smiles>

3ha

Colorless oil. $\quad R_{\mathrm{f}}=0.39($ EtOAc:hexane $=1: 20) . \quad$ Isolated yield is $57 \%(26.0 \mathrm{mg}) . \quad{ }^{1} \mathrm{H} \mathrm{NMR}(400 \mathrm{MHz}$, $\left.\mathrm{CDCl}_{3}\right): \delta 1.91-1.98(\mathrm{~m}, 2 \mathrm{H}), 2.60-2.68(\mathrm{~m}, 4 \mathrm{H}), 3.81(\mathrm{~s}, 3 \mathrm{H}), 6.85(\mathrm{~d}, J=8.8 \mathrm{~Hz}, 2 \mathrm{H}), 7.12(\mathrm{~d}, J=8.8 \mathrm{~Hz}, 2 \mathrm{H})$, 7.18-7.22 (m, 3H), 7.28-7.32 (m, 2H); ${ }^{13} \mathrm{C}\left\{{ }^{1} \mathrm{H}\right\} \mathrm{NMR}\left(101 \mathrm{MHz}, \mathrm{CDCl}_{3}\right): \delta 33.4,34.6,35.5,55.4,113.8,125.8$, $128.4,128.6,129.4,134.5,142.5,157.8$.

5-(3-Phenylpropyl)benzo[d][1,3]dioxole (3ia) ${ }^{7}$<smiles>c1ccc(CCCc2ccc3c(c2)OCO3)cc1</smiles>

3ia

Colorless oil. $\quad R_{\mathrm{f}}=0.44($ EtOAc:hexane $=1: 20) . \quad$ Isolated yield is $56 \%(26.8 \mathrm{mg}) . \quad{ }^{1} \mathrm{H}$ NMR $(400 \mathrm{MHz}$, $\left.\mathrm{CDCl}_{3}\right): \delta 1.88-1.96(\mathrm{~m}, 2 \mathrm{H}), 2.58(\mathrm{t}, J=7.6 \mathrm{~Hz}, 2 \mathrm{H}), 2.64(\mathrm{t}, J=7.6 \mathrm{~Hz}, 2 \mathrm{H}), 5.93(\mathrm{~s}, 2 \mathrm{H}), 6.62-6.75(\mathrm{~m}, 3 \mathrm{H})$, 7.18-7.21 (m, 3H), 7.27-7.30 (m, 2H); ${ }^{13} \mathrm{C}\left\{{ }^{1} \mathrm{H}\right\} \mathrm{NMR}\left(101 \mathrm{MHz}, \mathrm{CDCl}_{3}\right): \delta 33.4,35.3,35.4,100.9,108.2,109.0$, $121.3,125.9,128.4,128.6,136.3,142.4,145.6,147.6$.

\section{1-Fluoro-4-(3-phenylpropyl)benzene (3ja) ${ }^{2}$}<smiles>Fc1ccc(CCCc2ccccc2)cc1</smiles>

\section{3ja}

Colorless oil. $\quad R_{\mathrm{f}}=0.39$ (hexane). Isolated yield is $68 \%(29.0 \mathrm{mg}) . \quad{ }^{1} \mathrm{H} \mathrm{NMR}\left(400 \mathrm{MHz}, \mathrm{CDCl}_{3}\right): \delta 1.92-1.99$ (m, 2H), 2.62-2.68 (m, 4H), 6.96-7.00 (m, 2H), 7.13-7.16 (m, 2H), 7.19-7.23 (m, 3H), 7.29-7.33 (m, 2H); ${ }^{13} \mathrm{C}\left\{{ }^{1} \mathrm{H}\right\}$ $\operatorname{NMR}\left(101 \mathrm{MHz}, \mathrm{CDCl}_{3}\right): \delta 33.2,34.7,35.5,115.1\left(\mathrm{~d},{ }^{2} J_{\mathrm{C}-\mathrm{F}}=21 \mathrm{~Hz}\right), 125.9,128.5,128.6,129.8\left(\mathrm{~d},{ }^{3} J_{\mathrm{C}-\mathrm{F}}=8\right.$ $\mathrm{Hz}), 138.0,142.2,161.3\left(\mathrm{~d},{ }^{1} J_{\mathrm{C}-\mathrm{F}}=244 \mathrm{~Hz}\right) ;{ }^{19} \mathrm{~F}\left\{{ }^{1} \mathrm{H}\right\} \mathrm{NMR}\left(376 \mathrm{MHz}, \mathrm{CDCl}_{3}, \mathrm{rt}\right): \delta-117.9$. 
1-(3-Phenylpropyl)-2-(trifluoromethyl)benzene (3ka) ${ }^{8}$

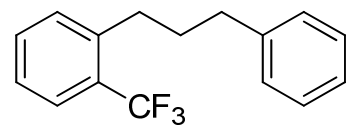

$3 \mathrm{ka}$

Colorless oil. $\quad R_{\mathrm{f}}=0.48$ (hexane). Isolated yield is $74 \%(38.9 \mathrm{mg}) . \quad{ }^{1} \mathrm{H}$ NMR $\left(600 \mathrm{MHz}, \mathrm{CDCl}_{3}\right): \delta 1.94-1.99$ $(\mathrm{m}, 2 \mathrm{H}), 2.73(\mathrm{t}, J=8.1 \mathrm{~Hz}, 2 \mathrm{H}), 2.83(\mathrm{t}, J=8.1 \mathrm{~Hz}, 2 \mathrm{H}), 7.19-7.22(\mathrm{~m}, 3 \mathrm{H}), 7.27-7.32(\mathrm{~m}, 4 \mathrm{H}), 7.46(\mathrm{t}, J=7.8$ $\mathrm{Hz}, 1 \mathrm{H}), 7.62(\mathrm{~d}, J=7.8 \mathrm{~Hz}, 1 \mathrm{H}) ;{ }^{13} \mathrm{C}\left\{{ }^{1} \mathrm{H}\right\} \mathrm{NMR}\left(151 \mathrm{MHz}, \mathrm{CDCl}_{3}\right): \delta 32.5,33.5,36.1,124.8\left(\mathrm{q},{ }^{1} J_{\mathrm{C}-\mathrm{F}}=274\right.$ $\mathrm{Hz}), 125.97,125.99,126.0\left(\mathrm{q},{ }^{3} J_{\mathrm{C}-\mathrm{F}}=6 \mathrm{~Hz}\right), 128.49,128.52,128.53\left(\mathrm{q},{ }^{2} J_{\mathrm{C}-\mathrm{F}}=29 \mathrm{~Hz}\right), 131.0,131.8,141.3$, $142.1 ;{ }^{19} \mathrm{~F}\left\{{ }^{1} \mathrm{H}\right\}$ NMR $\left(282 \mathrm{MHz}, \mathrm{CDCl}_{3}, \mathrm{rt}\right): \delta-59.7$.

1-(tert-Butyl)-4-(3-phenylpropyl)benzene (3la) ${ }^{9}$<smiles>FC(F)(F)c1ccc(CCCc2ccccc2)cc1</smiles>

3la

Colorless oil. $\quad R_{\mathrm{f}}=0.40$ (hexane). Isolated yield is $61 \%(32.2 \mathrm{mg}) . \quad{ }^{1} \mathrm{H} \mathrm{NMR}\left(400 \mathrm{MHz}, \mathrm{CDCl}_{3}\right): \delta 1.94-2.02$ (m, 2H), 2.64-2.73 (m, 4H), 7.18-7.22 (m, 3H), 7.28-7.32 (m, 4H), $7.54(\mathrm{~d}, J=8.0 \mathrm{~Hz}, 2 \mathrm{H}) ;{ }^{13} \mathrm{C}\left\{{ }^{1} \mathrm{H}\right\} \mathrm{NMR}(151$ $\left.\mathrm{MHz}_{\mathrm{CDCl}}\right): \delta 32.8,35.3,35.5,124.5\left(\mathrm{q},{ }^{1} J_{\mathrm{C}-\mathrm{F}}=271 \mathrm{~Hz}\right), 125.4\left(\mathrm{q},{ }^{3} J_{\mathrm{C}-\mathrm{F}}=4 \mathrm{~Hz}\right), 126.0,128.3\left(\mathrm{q},{ }^{2} J_{\mathrm{C}-\mathrm{F}}=32\right.$ $\mathrm{Hz}), 128.5,128.6,128.9,142.0,146.5 ;{ }^{19} \mathrm{~F}\left\{{ }^{1} \mathrm{H}\right\} \mathrm{NMR}\left(376 \mathrm{MHz}, \mathrm{CDCl}_{3}, \mathrm{rt}\right): \delta-62.3$.

4-(3-Phenylpropyl)benzonitrile (3ma) ${ }^{10}$<smiles>N#Cc1ccc(CCCc2ccccc2)cc1</smiles>

Colorless oil. $\quad R_{\mathrm{f}}=0.28($ EtOAc:hexane $=1: 20) . \quad$ Isolated yield is $68 \%(30.2 \mathrm{mg}) .{ }^{1} \mathrm{H} \mathrm{NMR}(600 \mathrm{MHz}$, $\left.\mathrm{CDCl}_{3}\right): \delta 1.94-1.99(\mathrm{~m}, 2 \mathrm{H}), 2.65(\mathrm{t}, J=7.8 \mathrm{~Hz}, 2 \mathrm{H}), 2.70(\mathrm{t}, J=7.8 \mathrm{~Hz}, 2 \mathrm{H}), 7.17-7.22(\mathrm{~m}, 3 \mathrm{H}), 7.27-7.31(\mathrm{~m}$, 
4H), 7.56-7.58 (m, 2H); ${ }^{13} \mathrm{C}\left\{{ }^{1} \mathrm{H}\right\}$ NMR (151 MHz, $\left.\mathrm{CDCl}_{3}\right): \delta 32.6,35.4,35.6,109.8,119.3,126.1,128.5,128.6$, $129.4,132.3,141.7,148.1$.

\section{4-Phenyl-(3-phenylpropyl)phenylmethanone (3na)}<smiles>O=C(c1ccccc1)c1ccc(CCCc2ccccc2)cc1</smiles>

Colorless oil. $\quad R_{\mathrm{f}}=0.25($ EtOAc:hexane $=1: 20) . \quad$ Isolated yield is $70 \%(42.3 \mathrm{mg}) . \quad{ }^{1} \mathrm{H}$ NMR $(400 \mathrm{MHz}$, $\left.\mathrm{CDCl}_{3}\right): \delta 1.99-2.06(\mathrm{~m}, 2 \mathrm{H}), 2.70(\mathrm{t}, J=7.8 \mathrm{~Hz}, 2 \mathrm{H}), 2.75(\mathrm{t}, J=7.8 \mathrm{~Hz}, 2 \mathrm{H}), 7.20-7.23(\mathrm{~m}, 3 \mathrm{H}), 7.30-7.33(\mathrm{~m}$, 4H), 7.47-7.51 (m, 2H), 7.57-7.61 (m, 1H), 7.75-7.80 (m, 2H), 7.81-7.83 (m, 2H); ${ }^{13} \mathrm{C}\left\{{ }^{1} \mathrm{H}\right\} \mathrm{NMR}(101 \mathrm{MHz}$, $\left.\mathrm{CDCl}_{3}\right): \delta 32.7,35.5,35.6,126.0,128.3,128.49$ (2C), 128.54, 130.1, 130.5, 132.3, 135.3, 138.0, 142.0, 147.6, 196.6. FT-IR (cm $\left.{ }^{-1}\right): 785$ (s), 849 (s), 972 (s), 1001 (s), 1177 (s), 1279 (s), 1308 (s), 1447 (s), 1566 (s), 1578 (s), 2936 (s), 3026 (s). Anal. Calcd for $\mathrm{C}_{22} \mathrm{H}_{20} \mathrm{O}$ : C, 87.96; H, 6.71\%. Found: C, 87.72; H, 6.81\%.

\section{1-(3-Phenylpropyl)naphthalene (3oa) $)^{11}$}<smiles>c1ccc(CCCc2cccc3ccccc23)cc1</smiles>

\section{3oa}

Colorless oil. $\quad R_{\mathrm{f}}=0.27$ (hexane). Isolated yield is $78 \%(38.5 \mathrm{mg}) . \quad{ }^{1} \mathrm{H} \mathrm{NMR}\left(400 \mathrm{MHz}, \mathrm{CDCl}_{3}\right): \delta 2.07-2.15$ (m, 2H), 2.77 (t, $J=7.8 \mathrm{~Hz}, 2 \mathrm{H}), 3.12(\mathrm{t}, J=7.8 \mathrm{~Hz}, 2 \mathrm{H}), 7.19-7.24(\mathrm{~m}, 3 \mathrm{H}), 7.29-7.34(\mathrm{~m}, 3 \mathrm{H}), 7.38-7.42(\mathrm{~m}$, 1H), 7.45-7.51 (m, 2H), $7.72(\mathrm{~d}, J=8.4 \mathrm{~Hz}, 1 \mathrm{H}), 7.84-7.87(\mathrm{~m}, 1 \mathrm{H}), 7.95-7.98(\mathrm{~m}, 1 \mathrm{H}) ;{ }^{13} \mathrm{C}\left\{{ }^{1} \mathrm{H}\right\} \mathrm{NMR}(101$ $\left.\mathrm{MHz}, \mathrm{CDCl}_{3}\right): \delta 32.4,32.7,36.0,123.9,125.5,125.7,125.8,125.9,126.1,126.7,128.5,128.6,128.9,132.0$, $134.0,138.5,142.3$.

\section{2-(3-Phenylpropyl)naphthalene (3pa) ${ }^{2}$}




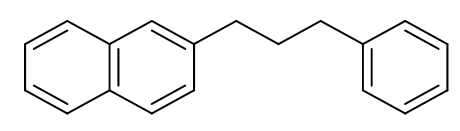

3pa

Colorless oil. $\quad R_{\mathrm{f}}=0.27$ (hexane). Isolated yield is $81 \%(39.7 \mathrm{mg}) . \quad{ }^{1} \mathrm{H} \mathrm{NMR}\left(400 \mathrm{MHz}, \mathrm{CDCl}_{3}\right): \delta 2.03-2.10$ (m, 2H), $2.70(\mathrm{t}, J=7.6 \mathrm{~Hz}, 2 \mathrm{H}), 2.83(\mathrm{t}, J=7.6 \mathrm{~Hz}, 2 \mathrm{H}), 7.20-7.23(\mathrm{~m}, 3 \mathrm{H}), 7.28-7.36(\mathrm{~m}, 3 \mathrm{H}), 7.41-7.79(\mathrm{~m}$, 2H), 7.63 (s, 1H), 7.77-7.83 (m, 3H); ${ }^{13} \mathrm{C}\left\{{ }^{1} \mathrm{H}\right\}$ NMR (101 MHz, $\left.\mathrm{CDCl}_{3}\right): \delta 33.0,35.6,35.7,125.2,125.9,126.0$, $126.6,127.51,127.54,127.7,128.0,128.5,128.6,132.1,133.7,139.9,142.4$.

\section{2-(3-Phenylpropyl)benzofuran (3qa) ${ }^{12}$}

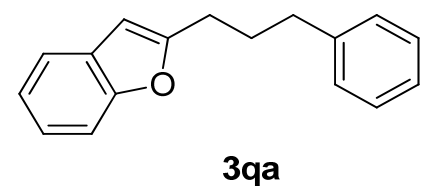

Pale yellow oil. $\quad R_{\mathrm{f}}=0.54($ EtOAc:hexane $=1: 20) . \quad$ Isolated yield is $54 \%(25.5 \mathrm{mg}) .{ }^{1} \mathrm{H}$ NMR $(400 \mathrm{MHz}$, $\left.\mathrm{CDCl}_{3}\right): \delta 2.06-2.14(\mathrm{~m}, 2 \mathrm{H}), 2.73(\mathrm{t}, J=7.8 \mathrm{~Hz}, 2 \mathrm{H}), 2.81(\mathrm{t}, J=7.8 \mathrm{~Hz}, 2 \mathrm{H}), 6.41(\mathrm{~s}, 1 \mathrm{H}), 7.17-7.24(\mathrm{~m}, 5 \mathrm{H})$, 7.29-7.33 (m, 2H), 7.41-7.43 (m, 1H), 7.48-7.50 (m, 1H); ${ }^{13} \mathrm{C}\left\{{ }^{1} \mathrm{H}\right\}$ NMR (101 MHz, $\left.\mathrm{CDCl}_{3}\right): \delta 28.0,29.4,35.3$, $102.3,110.9,120.3,122.5,123.3,126.1,128.5,128.7,129.1,141.9,154.8,159.3$.

\section{2-(3-Phenylpropyl)benzothiophene (3ra) ${ }^{13}$}

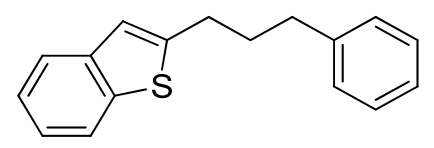

3ra

Pale yellow solid. $\quad R_{\mathrm{f}}=0.54$ (EtOAc:hexane $\left.=1: 20\right) . \quad$ Isolated yield is $64 \%(32.3 \mathrm{mg}) . \quad{ }^{1} \mathrm{H} \mathrm{NMR}(400 \mathrm{MHz}$, $\left.\mathrm{CDCl}_{3}\right): \delta 2.06-2.13(\mathrm{~m}, 2 \mathrm{H}), 2.73(\mathrm{t}, J=7.6 \mathrm{~Hz}, 2 \mathrm{H}), 2.94(\mathrm{t}, J=7.6 \mathrm{~Hz}, 2 \mathrm{H}), 7.02(\mathrm{~s}, 1 \mathrm{H}), 7.19-7.25(\mathrm{~m}, 4 \mathrm{H})$, 7.27-7.33 (m, 3H), 7.66-7.68 (m, 1H), 7.76-7.78 (m, 1H); ${ }^{13} \mathrm{C}\left\{{ }^{1} \mathrm{H}\right\}$ NMR (101 MHz, $\left.\mathrm{CDCl}_{3}\right): \delta 30.4,32.8,35.3$, $120.9,122.3,122.8,123.6,124.2,126.0,128.5,128.6,139.5,140.3,141.9,146.3$.

\section{4-Methyl-(3-phenylpropyl) benzoate (3sa) ${ }^{14}$}


<smiles>COC(=O)c1ccc(CCCc2ccccc2)cc1</smiles>

Colorless oil. $\quad R_{\mathrm{f}}=0.28($ EtOAc:hexane $=1: 20) . \quad$ Isolated yield is $78 \%(39.6 \mathrm{mg}) .{ }^{1} \mathrm{H} \mathrm{NMR}(600 \mathrm{MHz}$, $\left.\mathrm{CDCl}_{3}\right): \delta 1.95-2.00(\mathrm{~m}, 2 \mathrm{H}), 2.65(\mathrm{t}, J=7.8 \mathrm{~Hz}, 2 \mathrm{H}), 2.70(\mathrm{t}, J=7.8 \mathrm{~Hz}, 2 \mathrm{H}), 3.91(\mathrm{~s}, 3 \mathrm{H}), 7.17-7.21(\mathrm{~m}, 3 \mathrm{H})$, 7.25-7.28 (m, 2H), 7.29-7.31 (m, 2H), 7.95-7.97 (m, 2H); ${ }^{13} \mathrm{C}\left\{{ }^{1} \mathrm{H}\right\} \mathrm{NMR}\left(151 \mathrm{MHz}, \mathrm{CDCl}_{3}\right): \delta 32.8,35.5,35.6$, $52.1,126.0,127.9,128.5,128.6,128.6,129.8,142.0,148.0,167.3$.

\section{4-Phenyl-(3-phenylpropyl)benzoate (3ta)}<smiles>O=C(Oc1ccccc1)c1ccc(CCCc2ccccc2)cc1</smiles>

Colorless oil. $\quad R_{\mathrm{f}}=0.29($ EtOAc:hexane $=1: 20) . \quad$ Isolated yield is $65 \%(41.0 \mathrm{mg}) . \quad{ }^{1} \mathrm{H}$ NMR $(400 \mathrm{MHz}$, $\left.\mathrm{CDCl}_{3}\right): \delta 1.98-2.05(\mathrm{~m}, 2 \mathrm{H}), 2.68(\mathrm{t}, J=7.6 \mathrm{~Hz}, 2 \mathrm{H}), 2.75(\mathrm{t}, J=7.6 \mathrm{~Hz}, 2 \mathrm{H}), 7.19-7.23(\mathrm{~m}, 5 \mathrm{H}), 7.27-7.34(\mathrm{~m}$, 5H), 7.42-7.46 (m, 2H), 8.12-8.15 (m, 2H); ${ }^{13} \mathrm{C}\left\{{ }^{1} \mathrm{H}\right\} \mathrm{NMR}\left(101 \mathrm{MHz}, \mathrm{CDCl}_{3}\right): \delta 32.7,35.5,35.6,121.9,125.9$, 126.0, 127.3, 128.5, 128.6, 128.8, 129.6, 130.4, 142.0, 148.8, 151.1, 165.3. FT-IR ( $\left.\mathrm{cm}^{-1}\right): 700(\mathrm{~s}), 745(\mathrm{~s}), 1018$ (s), 1070 (s), 1177 (s), 1198 (s), 1267 (s), 1493 (s), 1736 (s). Anal. Calcd for $\mathrm{C}_{22} \mathrm{H}_{20} \mathrm{O}_{2}$ : C, 83.51; H, 6.37\%. Found: C, $83.51 ; \mathrm{H}, 6.40 \%$.

\section{1,2-Diphenylethane (3ab) ${ }^{15}$}<smiles>c1ccc(CCc2ccccc2)cc1</smiles>

$3 a b$

White solid. $\quad R_{\mathrm{f}}=0.44$ (hexane). Isolated yield is $80 \%(29.2 \mathrm{mg}) .{ }^{1} \mathrm{H}$ NMR $\left(400 \mathrm{MHz}, \mathrm{CDCl}_{3}\right): \delta 2.93(\mathrm{~s}, 4 \mathrm{H})$, 7.19-7.22 (m, 6H), 7.27-7.31 (m, 4H); ${ }^{13} \mathrm{C}\left\{{ }^{1} \mathrm{H}\right\} \mathrm{NMR}\left(101 \mathrm{MHz}, \mathrm{CDCl}_{3}\right): \delta 38.1,126.1,128.5,128.6,141.9$. 


\section{1,4-Diphenylbutane (3ac) ${ }^{15}$}<smiles>c1ccc(CCCCc2ccccc2)cc1</smiles>

3ac

Colorless oil. $\quad R_{\mathrm{f}}=0.44$ (hexane). Isolated yield is $81 \%(34.2 \mathrm{mg}) . \quad{ }^{1} \mathrm{H} \mathrm{NMR}\left(400 \mathrm{MHz}, \mathrm{CDCl}_{3}\right): \delta 1.68-1.71$ (m, 4H), 2.64-2.68 (m, 4H), 7.18-7.22 (m, 6H), 7.27-7.32 (m, 4H); ${ }^{13} \mathrm{C}\left\{{ }^{1} \mathrm{H}\right\}$ NMR (101 MHz, $\left.\mathrm{CDCl}_{3}\right): \delta 31.3$, $36.0,125.8,128.4,128.6,142.7$.

\section{1-Octylbenzene (3ad) ${ }^{16}$}

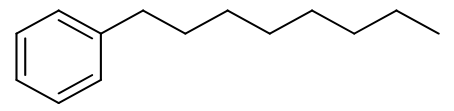

3ad

Colorless oil. $\quad R_{\mathrm{f}}=0.73$ (hexane). Isolated yield is $54 \%(20.7 \mathrm{mg}) . \quad{ }^{1} \mathrm{H} \mathrm{NMR}\left(400 \mathrm{MHz}, \mathrm{CDCl}_{3}\right): \delta 0.86-0.90$ (m, 3H), 1.27-1.31 (m, 10H), 1.57-1.65 (m, 2H), 2.60 (t, $J=7.8 \mathrm{~Hz}, 2 \mathrm{H}), 7.16-7.19(\mathrm{~m}, 3 \mathrm{H}), 7.28-7.30(\mathrm{~m}, 2 \mathrm{H})$; ${ }^{13} \mathrm{C}\left\{{ }^{1} \mathrm{H}\right\}$ NMR (101 MHz, $\left.\mathrm{CDCl}_{3}\right): \delta 14.3,22.8,29.4,29.5,29.6,31.7,32.1,36.2,125.7,128.4,128.5,143.1$.

\section{1-Dodecylbenzene (3ae) $)^{17}$}

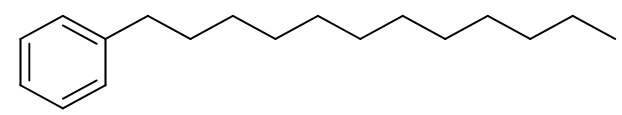

$3 a e$

Colorless oil. $\quad R_{\mathrm{f}}=0.73$ (hexane). Isolated yield is $62 \%(30.4 \mathrm{mg}) . \quad{ }^{1} \mathrm{H} \mathrm{NMR}\left(400 \mathrm{MHz}, \mathrm{CDCl}_{3}\right): \delta 0.87-0.91$ (m, 3H), 1.26-1.31 (m, 18H), 1.58-1.65 (m, 2H), 2.60 (t, $J=7.8 \mathrm{~Hz}, 2 \mathrm{H}), 7.16-7.19(\mathrm{~m}, 3 \mathrm{H}), 7.28-7.30(\mathrm{~m}, 2 \mathrm{H})$; ${ }^{13} \mathrm{C}\left\{{ }^{1} \mathrm{H}\right\}$ NMR (151 MHz, $\left.\mathrm{CDCl}_{3}\right): \delta 14.3,22.9,29.51,29.52,29.7,29.76,29.80,29.83,29.84,31.7,32.1,36.1$, $125.7,128.3,128.5,143.1$.

\section{2-Cyclohexylethylbenzene (3af) ${ }^{17}$}


<smiles>c1ccc(CCC2CCCCC2)cc1</smiles>

3af

Colorless oil. $\quad R_{\mathrm{f}}=0.73$ (hexane). Isolated yield is $79 \%(29.7 \mathrm{mg}) . \quad{ }^{1} \mathrm{H} \mathrm{NMR}\left(400 \mathrm{MHz}, \mathrm{CDCl}_{3}\right): \delta 0.90-0.98$ (m, 2H), 1.15-1.27 (m, 4H), 1.49-1.54 (m, 2H), 1.65-1.80 (m, 5H), 2.63 (t, $J=8.2 \mathrm{~Hz}, 2 \mathrm{H}), 7.16-7.20$ (m, 3H), 7.27-7.31 (m, 2H); ${ }^{13} \mathrm{C}\left\{{ }^{1} \mathrm{H}\right\}$ NMR (101 MHz, $\left.\mathrm{CDCl}_{3}\right): \delta 26.5,26.9,33.4,33.5,37.5,39.6,125.6,128.4,128.5$, 143.4.

1-Methoxy-4-(3-phenylpropyl)benzene (3ha)<smiles>COc1ccc(CCCc2ccccc2)cc1</smiles>

3ha

Colorless oil. $\quad R_{\mathrm{f}}=0.39$ (EtOAc:hexane $\left.=1: 20\right) . \quad$ Isolated yield is $82 \%(37.3 \mathrm{mg})$. The NMR data is shown in the reaction of $\mathbf{1 h}$ with $\mathbf{2 a}$.

5-(3-Phenylpropyl)benzo[d][1,3]dioxole (3ia)<smiles>c1ccc(CCCc2ccc3c(c2)OCO3)cc1</smiles>

3ia

Colorless oil. $\quad R_{\mathrm{f}}=0.44$ (EtOAc:hexane $\left.=1: 20\right) . \quad$ Isolated yield is $91 \%(43.7 \mathrm{mg}) . \quad$ The NMR data is shown in the reaction of $\mathbf{1 i}$ with $\mathbf{2 a}$.

3-Phenylpropylpentafluorobenzene (3ag) ${ }^{18}$ 
<smiles>Fc1c(F)c(F)c(CCCc2ccccc2)c(F)c1F</smiles>

Colorless oil. $\quad R_{\mathrm{f}}=0.47$ (hexane). Isolated yield is $72 \%(41.0 \mathrm{mg}) . \quad{ }^{1} \mathrm{H} \mathrm{NMR}\left(600 \mathrm{MHz}, \mathrm{CDCl}_{3}\right): \delta 1.90-1.95$ (m, 2H), $2.68(\mathrm{t}, J=7.5 \mathrm{~Hz}, 2 \mathrm{H}), 2.74(\mathrm{t}, J=7.5 \mathrm{~Hz}, 2 \mathrm{H}), 7.18-7.21(\mathrm{~m}, 3 \mathrm{H}), 7.28-7.31(\mathrm{~m}, 2 \mathrm{H}) ;{ }^{13} \mathrm{C}\left\{{ }^{1} \mathrm{H}\right\} \mathrm{NMR}$ $\left(151 \mathrm{MHz}, \mathrm{CDCl}_{3}\right): \delta 22.2,30.8,35.5,115.1-115.3(\mathrm{~m}), 126.2,128.4,128.6,137.5\left(\mathrm{dm},{ }^{1} J_{\mathrm{C}-\mathrm{F}}=252 \mathrm{~Hz}\right), 139.6$ $\left(\mathrm{dm},{ }^{1} J_{\mathrm{C}-\mathrm{F}}=252 \mathrm{~Hz}\right), 141.3,145.1\left(\mathrm{dm},{ }^{1} J_{\mathrm{C}-\mathrm{F}}=245 \mathrm{~Hz}\right) ;{ }^{19} \mathrm{~F}\left\{{ }^{1} \mathrm{H}\right\}$ NMR $\left(376 \mathrm{MHz}, \mathrm{CDCl}_{3}, \mathrm{rt}\right): \delta-162.9(\mathrm{td}, J=$ 21.6, 7.1 Hz, 2F), $-158.0(\mathrm{t}, J=20.8 \mathrm{~Hz}, 1 \mathrm{~F}),-144.2(\mathrm{dd}, J=22.2,8.4 \mathrm{~Hz}, 2 \mathrm{~F})$.

\section{6-Phenylmethylhexanoate (3ah) ${ }^{19}$}<smiles>COC(=O)CCCCCc1ccccc1</smiles>

3ah

Colorless oil. $\quad R_{\mathrm{f}}=0.28($ EtOAc:hexane $=1: 20) . \quad$ Isolated yield is $76 \%(31.2 \mathrm{mg}) . \quad{ }^{1} \mathrm{H} \mathrm{NMR}(600 \mathrm{MHz}$, $\left.\mathrm{CDCl}_{3}\right): \delta 1.34-1.39(\mathrm{~m}, 2 \mathrm{H}), 1.61-1.69(\mathrm{~m}, 4 \mathrm{H}), 2.31$ (t, $\left.J=7.8 \mathrm{~Hz}, 2 \mathrm{H}\right), 2.61(\mathrm{t}, J=7.8 \mathrm{~Hz}, 2 \mathrm{H}), 3.66(\mathrm{~s}, 3 \mathrm{H})$, 7.16-7.19 (m, 3H), 7.26-7.29 (m, 2H); ${ }^{13} \mathrm{C}\left\{{ }^{1} \mathrm{H}\right\} \mathrm{NMR}\left(151 \mathrm{MHz}, \mathrm{CDCl}_{3}\right): \delta$ 25.0, 28.9, 31.2, 34.2, 35.9, 51.6, $125.8,128.4,128.5,142.6,174.4$.

\section{6-Phenylhexyloxy-(tert-butyl)dimethylsilane (3ai) ${ }^{20}$

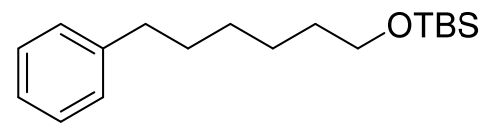

3ai

Colorless oil. $\quad R_{\mathrm{f}}=0.32($ EtOAc:hexane $=1: 20) . \quad$ Isolated yield is $85 \%(49.7 \mathrm{mg}) . \quad{ }^{1} \mathrm{H} \mathrm{NMR}(400 \mathrm{MHz}$, $\left.\mathrm{CDCl}_{3}\right): \delta 0.04$ (s, $\left.6 \mathrm{H}\right), 0.89$ (s, 9H), 1.33-1.36 (m, 4H), 1.48-1.64 (m, 4H), 2.60 (t, $\left.J=7.8 \mathrm{~Hz}, 2 \mathrm{H}\right), 3.59$ (t, $J=$ $6.6 \mathrm{~Hz}, 2 \mathrm{H}), 7.15-7.19(\mathrm{~m}, 3 \mathrm{H}), 7.27-7.28(\mathrm{~m}, 2 \mathrm{H}) ;{ }^{13} \mathrm{C}\left\{{ }^{1} \mathrm{H}\right\}$ NMR (101 MHz, $\left.\mathrm{CDCl}_{3}\right): \delta-5.1,18.5,25.8,26.1$, $29.3,31.7,32.9,36.1,63.4,125.7,128.4,128.5,143.0$. 


\section{Limitation of Substrates}<smiles>CN(C)c1ccc(CCCc2ccccc2)cc1</smiles>

$0 \%$<smiles>O=[N+]([O-])c1ccc(CCCc2ccccc2)cc1</smiles>

$0 \%$<smiles>C(=C/c1ccccc1)\CCCc1ccccc1</smiles>

$0 \%$<smiles>c1ccc(C2CCCCC2)cc1</smiles>

$3 \%$<smiles>Brc1ccc(CCCc2ccccc2)cc1</smiles>

$0 \%$<smiles>c1ccc(CCCCc2ccc3ccccc3c2)cc1</smiles>

$0 \%$<smiles>CCCONS(=O)(=O)c1ccc(CCCc2ccc3c(c2)OCO3)cc1</smiles>

From Probonecid $23 \%$<smiles>c1ccc(C2CCCCCCC2)cc1</smiles>

$9 \%$ 


\subsection{Gram-Scale Experiment.}

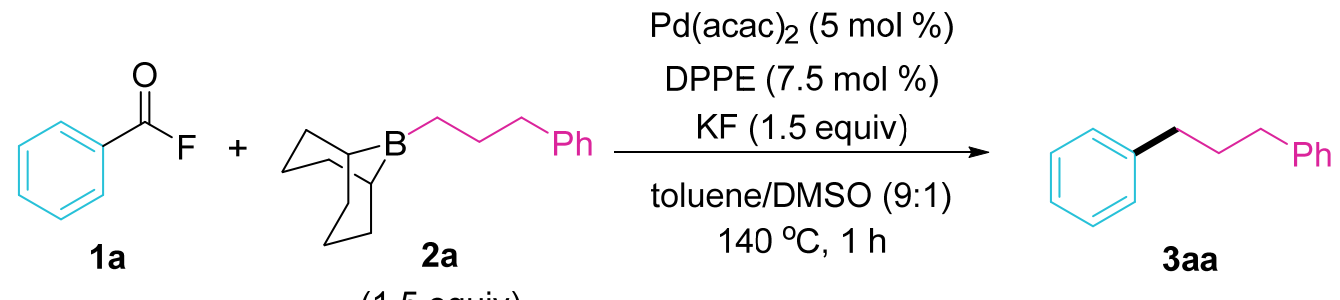

(1.5 equiv)

An oven-dried two-necked flask $(200 \mathrm{~mL})$ containing a magnetic stirring bar was charged with $\mathrm{Pd}(\mathrm{acac})_{2}$ (122.9 mg, $0.4 \mathrm{mmol}, 5 \mathrm{~mol} \%$ ), DPPE (239.1 mg, $0.6 \mathrm{mmol}, 7.5 \mathrm{~mol} \%$ ), KF (697.2 mg, $12 \mathrm{mmol}, 1.5$ equiv), toluene $(24 \mathrm{~mL})$, and DMSO $(4 \mathrm{~mL})$ under argon and the reaction mixture was stirred for $30 \mathrm{sec}$ at room temperature. After the solution of alkyl 9-BBN 2a (12 mL/1.0 M, 1.5 equiv), derived from the reaction of 9BBN-dimer (1.0 equiv) and allylbenzene (2.0 equiv) in dry toluene at $80^{\circ} \mathrm{C}$ for $3 \mathrm{~h}$, was added into the Schlenk tube, benzoyl fluoride (1a) $\left(992.9 \mathrm{mg}, 8 \mathrm{mmol}, 1.0\right.$ equiv) was added. The mixture was heated at $140{ }^{\circ} \mathrm{C}$ with stirring for $1 \mathrm{~h}$. After the mixture was cooled to room temperature, the mixture was quenched with saturated $\mathrm{NH}_{4} \mathrm{Cl}$ and the aqueous solution was extracted with $\mathrm{Et}_{2} \mathrm{O}$. The combined organic extracts were dried over anhydrous $\mathrm{MgSO}_{4}$, and filtered and evaporated under vacuum to give the crude product which was purified by column chromatography (hexane) on silica gel to afford the desired product 3aa (925 mg, $4.71 \mathrm{mmol}$ ) in 59\% yield.

\subsection{Alkylation of 3ta: Formation of 5.}

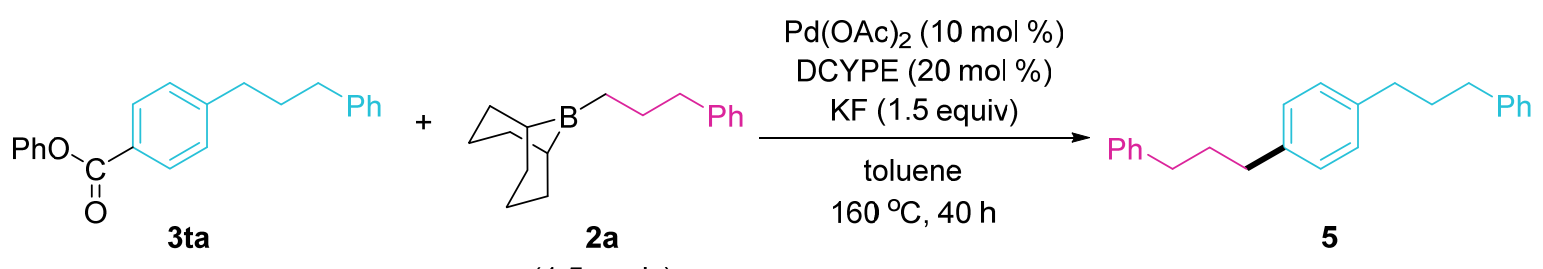

(1.5 equiv)

The reaction was conducted according to the reported procedure. ${ }^{14}$ An oven-dried Schlenk tube $(25 \mathrm{~mL})$ containing a magnetic stirring bar was charged with $\mathrm{Pd}(\mathrm{OAc})_{2}(4.6 \mathrm{mg}, 0.02 \mathrm{mmol}, 10 \mathrm{~mol} \%)$, DCYPE (16.8 $\mathrm{mg}, 0.04 \mathrm{mmol}, 20 \mathrm{~mol} \%), \mathrm{KF}(17.4 \mathrm{mg}, 0.3 \mathrm{mmol}, 1.5$ equiv), and toluene $(0.6 \mathrm{~mL})$ under argon and the reaction mixture was stirred for $30 \mathrm{sec}$ at room temperature. After the solution of alkyl 9-BBN $2 \mathrm{a}(0.3 \mathrm{~mL} / 1.0$ 
$\mathrm{M}, 1.5$ equiv), derived from the reaction of 9-BBN-dimer (1.0 equiv) and allylbenzene ( 2.0 equiv) in dry toluene at $80{ }^{\circ} \mathrm{C}$ for $3 \mathrm{~h}$, was added into the Schelenk tube, and sequentially 3 ta $(63.3 \mathrm{mg}, 0.2 \mathrm{mmol}, 1.0$ equiv) was added. The mixture was heated at $160{ }^{\circ} \mathrm{C}$ with stirring for $40 \mathrm{~h}$. After the mixture was cooled to room temperature, quenched with saturated $\mathrm{NH}_{4} \mathrm{Cl}$, and extracted with $\mathrm{Et}_{2} \mathrm{O}$. The combined organic extracts were dried over anhydrous $\mathrm{MgSO}_{4}$, and filtered and evaporated under vacuum to afford the crude product which was purified by column chromatography on silica gel to afford $5^{21}(44.5 \mathrm{mg})$ in $71 \%$ yield. Colorless oil. $R_{\mathrm{f}}=0.25$ (hexane). ${ }^{1} \mathrm{H}$ NMR (400 MHz, $\left.\mathrm{CDCl}_{3}\right): \delta$ 1.91-1.99 (m, 4H), 2.61-2.67 (m, 8H), $7.11(\mathrm{~s}, 4 \mathrm{H}), 7.16-7.20(\mathrm{~m}, 6 \mathrm{H})$, $7.28(\mathrm{t}, J=7.4 \mathrm{~Hz}, 4 \mathrm{H}) ;{ }^{13} \mathrm{C}\left\{{ }^{1} \mathrm{H}\right\} \mathrm{NMR}\left(101 \mathrm{MHz}, \mathrm{CDCl}_{3}\right): \delta 33.2,35.2,35.6,125.8,128.4,128.5,128.6,139.7$, 142.5 .

\subsection{Decarbonylative Alkylation of Acyl Fluorides under Previously Reported Catalytic Systems.}

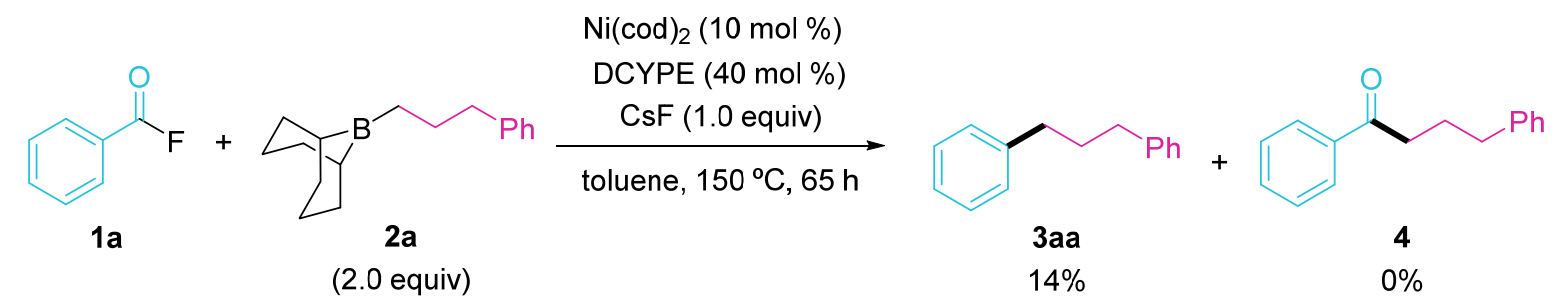

The following reaction was conducted according to the reported procedure. ${ }^{12}$ An oven-dried Schlenk tube $(25 \mathrm{~mL})$ containing a magnetic stirring bar was charged with $\mathrm{Ni}(\operatorname{cod})_{2}(5.5 \mathrm{mg}, 0.02 \mathrm{mmol}, 10 \mathrm{~mol} \%)$, DCYPE (33.6 $\mathrm{mg}, 0.08 \mathrm{mmol}, 40 \mathrm{~mol} \%), \mathrm{CsF}(15.2 \mathrm{mg}, 0.2 \mathrm{mmol}, 1.0$ equiv), and toluene ( $0.6 \mathrm{~mL}$ ) under argon and the mixture was stirred for $30 \mathrm{sec}$ at room temperature. After the solution of alkyl 9-BBN $2 \mathrm{a}(0.4 \mathrm{~mL} / 1.0 \mathrm{M}, 2.0$ equiv), derived from the reaction of 9-BBN-dimer (1.0 equiv) and allybenzene (2.0 equiv) in dry toluene at $80{ }^{\circ} \mathrm{C}$ for $3 \mathrm{~h}$, was added into the Schlenk tube, benzoyl fluoride (1a) $(24.8 \mathrm{mg}, 0.2 \mathrm{mmol}, 1.0$ equiv) was added. The mixture was heated at $150{ }^{\circ} \mathrm{C}$ with stirring for $65 \mathrm{~h}$. After the mixture was cooled to room temperature, the mixture was quenched with saturated $\mathrm{NH}_{4} \mathrm{Cl}$ and the aqueous solution was extracted with $\mathrm{Et}_{2} \mathrm{O}$. The combined organic extracts were dried over anhydrous $\mathrm{MgSO}_{4}$, filtered, and evaporated under vacuum to give the crude product. The GC yield was calculated using $n$-dodecane as an internal standard. 


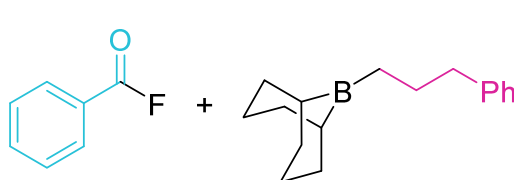

$1 a$

$2 a$

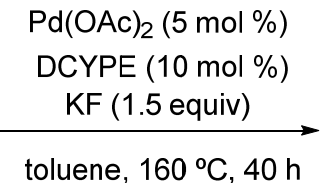

(2.0 equiv)

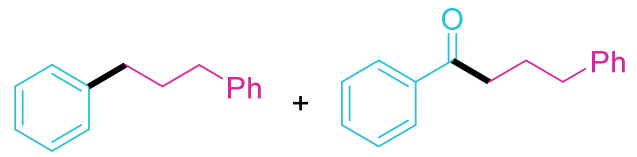

3aa

$19 \%$
4

$1 \%$

The following reaction was conducted according to the reported procedure. ${ }^{14}$ An oven-dried Schlenk tube $(25 \mathrm{~mL})$ containing a magnetic stirring bar was charged with $\mathrm{Pd}(\mathrm{OAc})_{2}(2.3 \mathrm{mg}, 0.01 \mathrm{mmol}, 5 \mathrm{~mol} \%)$, DCYPE (8.4 mg, $0.02 \mathrm{mmol}, 10 \mathrm{~mol} \%), \mathrm{KF}(17.4 \mathrm{mg}, 0.3 \mathrm{mmol}, 1.5$ equiv), toluene $(0.6 \mathrm{~mL})$ under argon and the reaction mixture was stirred for $30 \mathrm{sec}$ at room temperature. After the solution of alkyl 9-BBN $2 \mathrm{a}(0.3 \mathrm{~mL} / 1.0$ $\mathrm{M}, 1.5$ equiv), derived from the reaction of 9-BBN-dimer (1.0 equiv) and allylbenzene ( 2.0 equiv) in dry toluene at $80{ }^{\circ} \mathrm{C}$ for $3 \mathrm{~h}$, was added into the Schlenk tube, benzoyl fluoride (1a) $(24.8 \mathrm{mg}, 0.2 \mathrm{mmol}, 1.0$ equiv) was added. The mixture was heated at $160{ }^{\circ} \mathrm{C}$ with stirring for $40 \mathrm{~h}$. After the mixture was cooled to room temperature, the mixture was quenched with saturated $\mathrm{NH}_{4} \mathrm{Cl}$ and the aqueous solution was extracted with $\mathrm{Et}_{2} \mathrm{O}$. The combined organic extracts were dried over anhydrous $\mathrm{MgSO}_{4}$, filtered, and evaporated under vacuum to give the crude product. The GC yield was calculated using $n$-dodecane as an internal standard. 
4. Copies of ${ }^{1} \mathrm{H},{ }^{13} \mathrm{C}\left\{{ }^{1} \mathrm{H}\right\}$, and ${ }^{19} \mathrm{~F}\left\{{ }^{1} \mathrm{H}\right\}$ NMR Charts for the Products
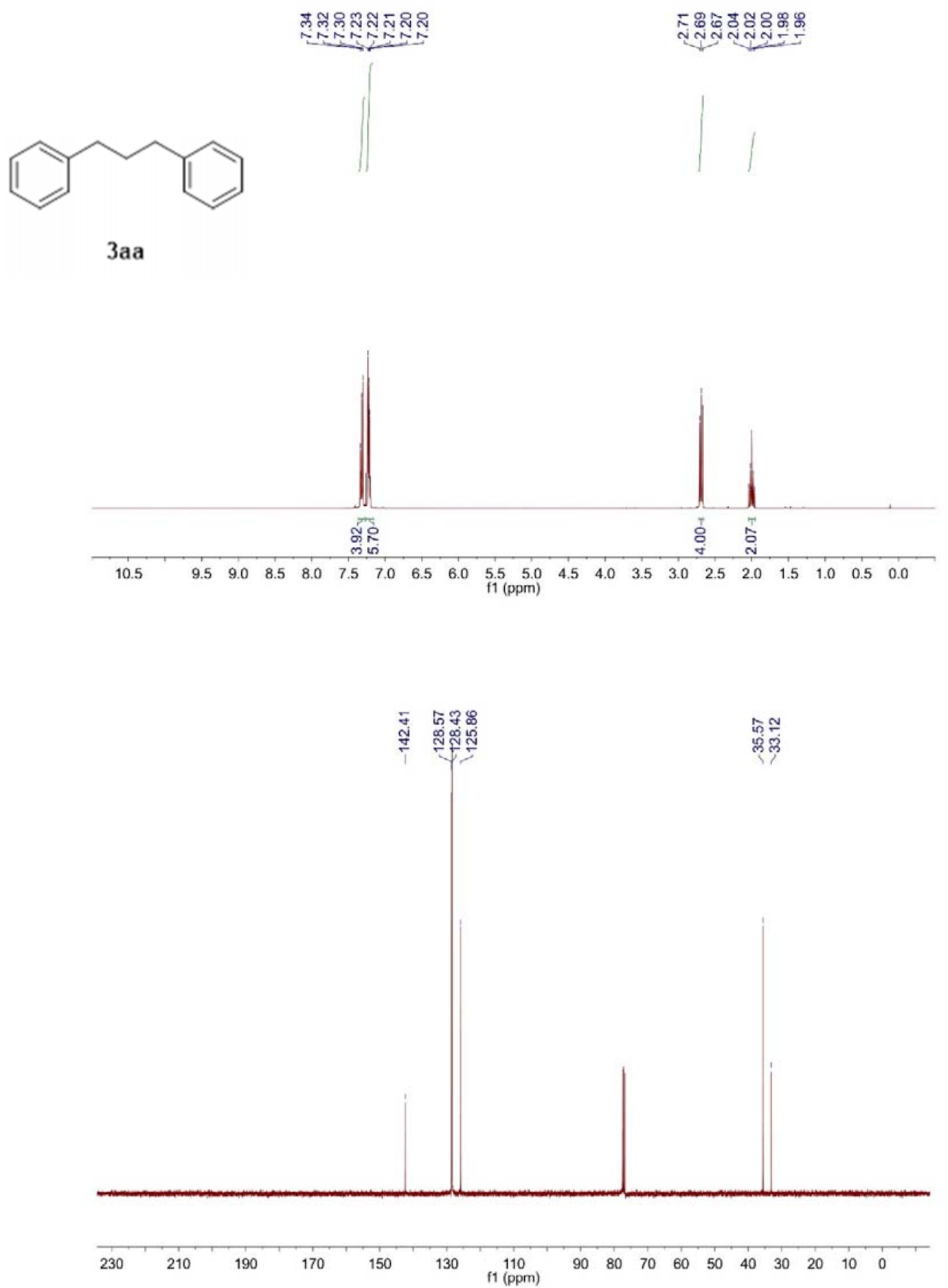

${ }^{1} \mathrm{H}$ NMR (400 MHz) and ${ }^{13} \mathrm{C}\left\{{ }^{1} \mathrm{H}\right\}$ NMR (101 MHz) spectra of 3aa (rt, $\left.\mathrm{CDCl}_{3}\right)$. 


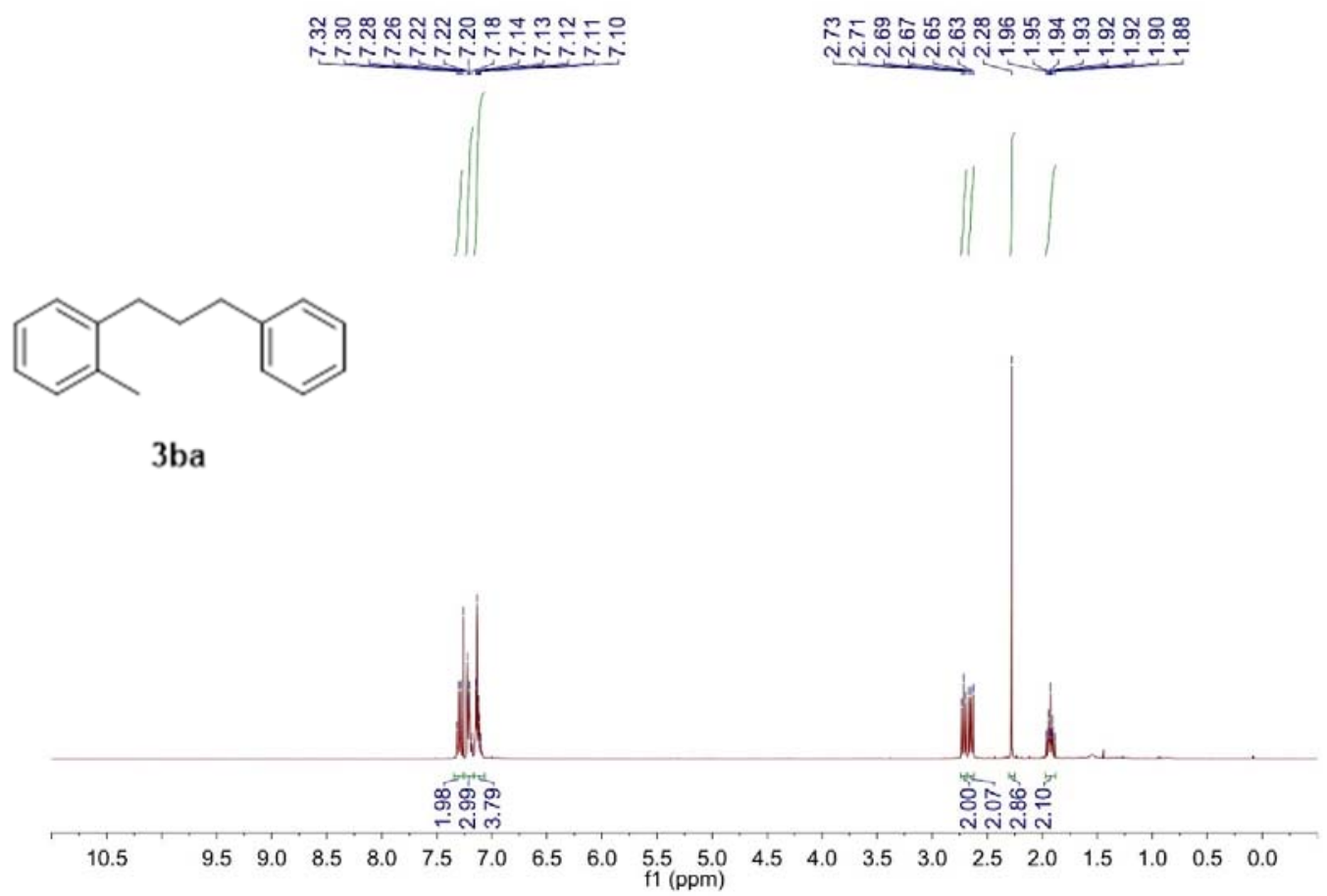

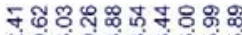

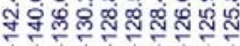

ด्रूक ₹

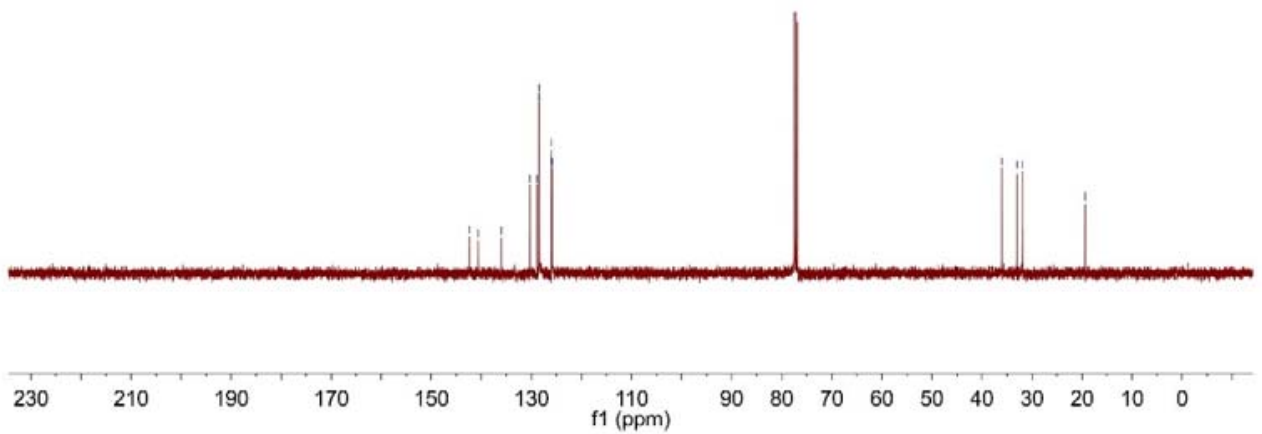

${ }^{1} \mathrm{H}$ NMR $(400 \mathrm{MHz})$ and ${ }^{13} \mathrm{C}\left\{{ }^{1} \mathrm{H}\right\}$ NMR (101 MHz) spectra of 3ba (rt, $\left.\mathrm{CDCl}_{3}\right)$. 


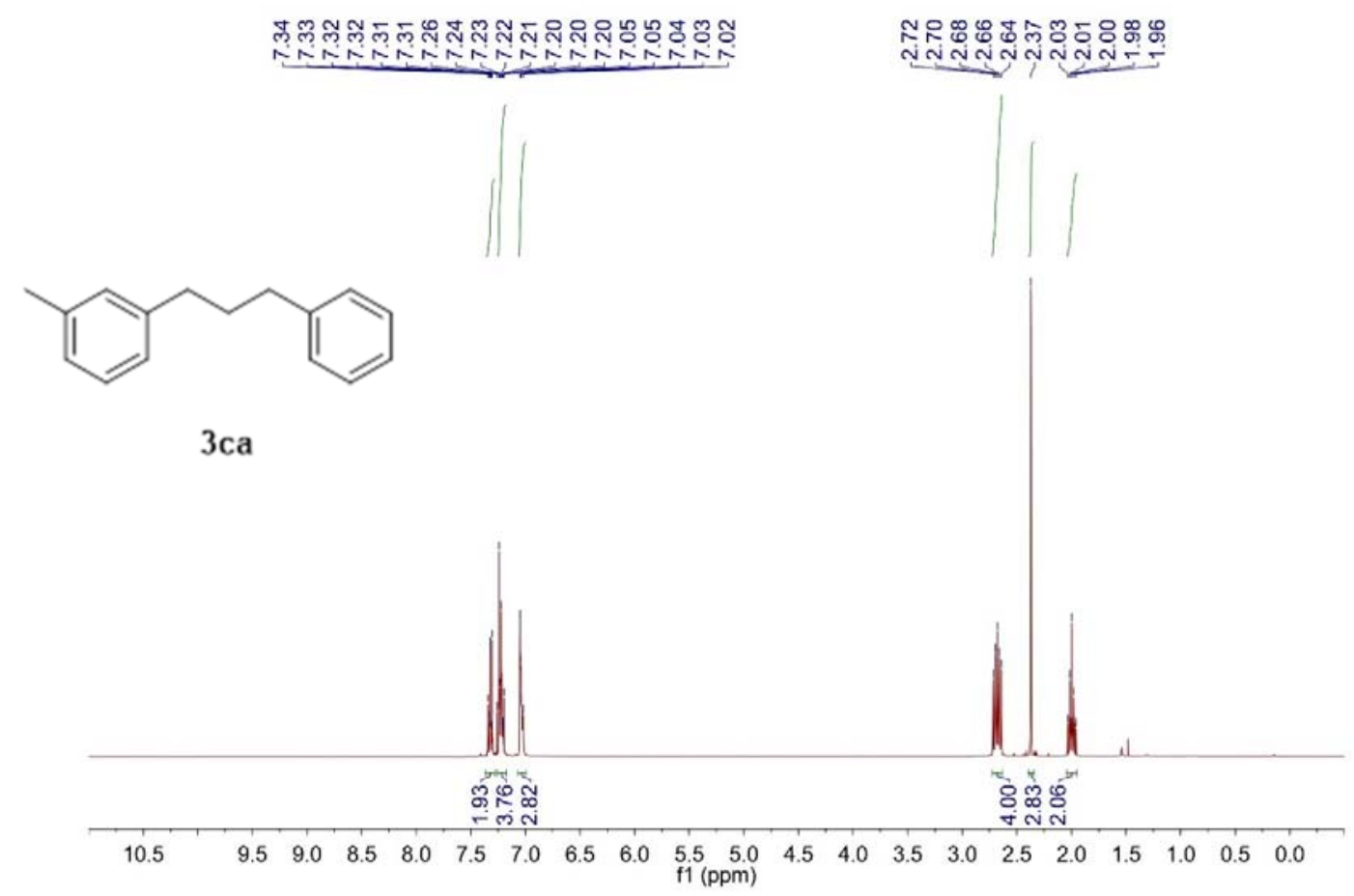

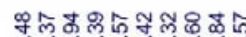
ปู่

ณํㅜㅇ

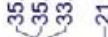

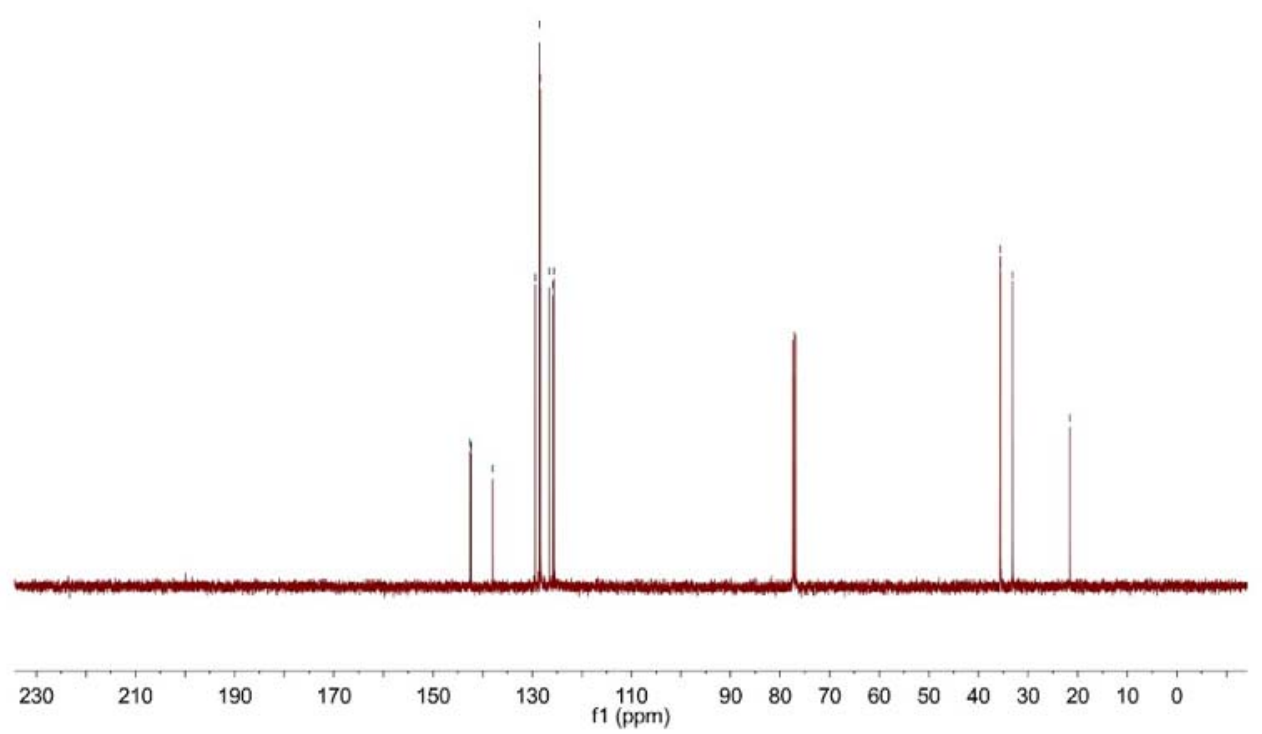

${ }^{1} \mathrm{H}$ NMR (400 MHz) and ${ }^{13} \mathrm{C}\left\{{ }^{1} \mathrm{H}\right\}$ NMR (101 MHz) spectra of 3ca (rt, $\left.\mathrm{CDCl}_{3}\right)$. 


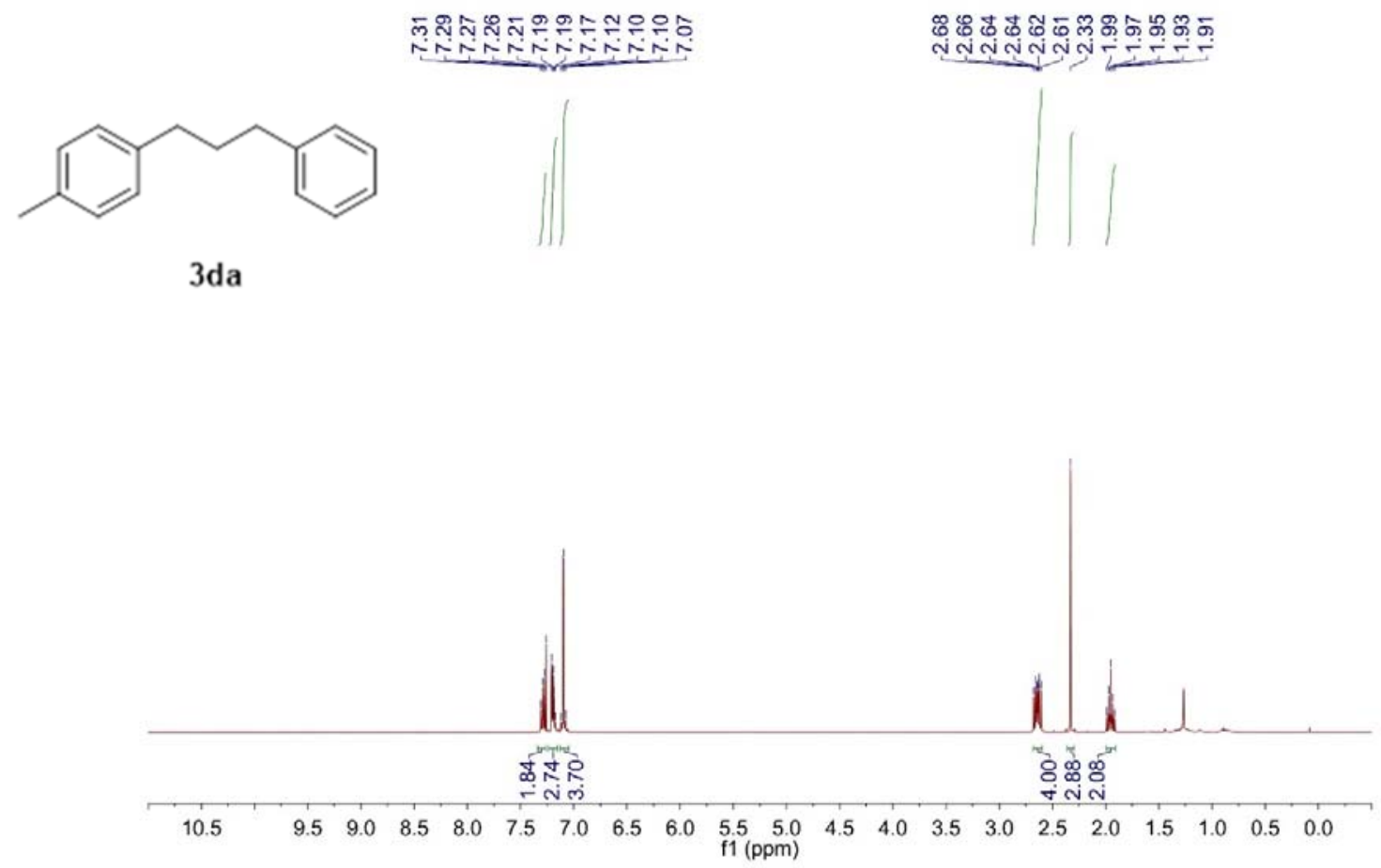

Вำำำ

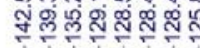

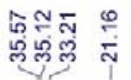

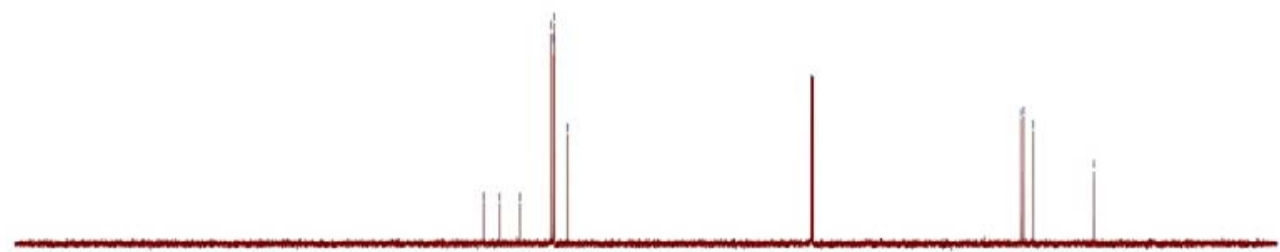

$\begin{array}{llllllllllllllllllllllllll}230 & 210 & 190 & 170 & 150 & 130 & 110 & 90 & 80 & 70 & 60 & 50 & 40 & 30 & 20 & 10 & 0 & \end{array}$

${ }^{1} \mathrm{H}$ NMR (400 MHz) and ${ }^{13} \mathrm{C}\left\{{ }^{1} \mathrm{H}\right\}$ NMR (151 MHz) spectra of 3da (rt, $\left.\mathrm{CDCl}_{3}\right)$. 


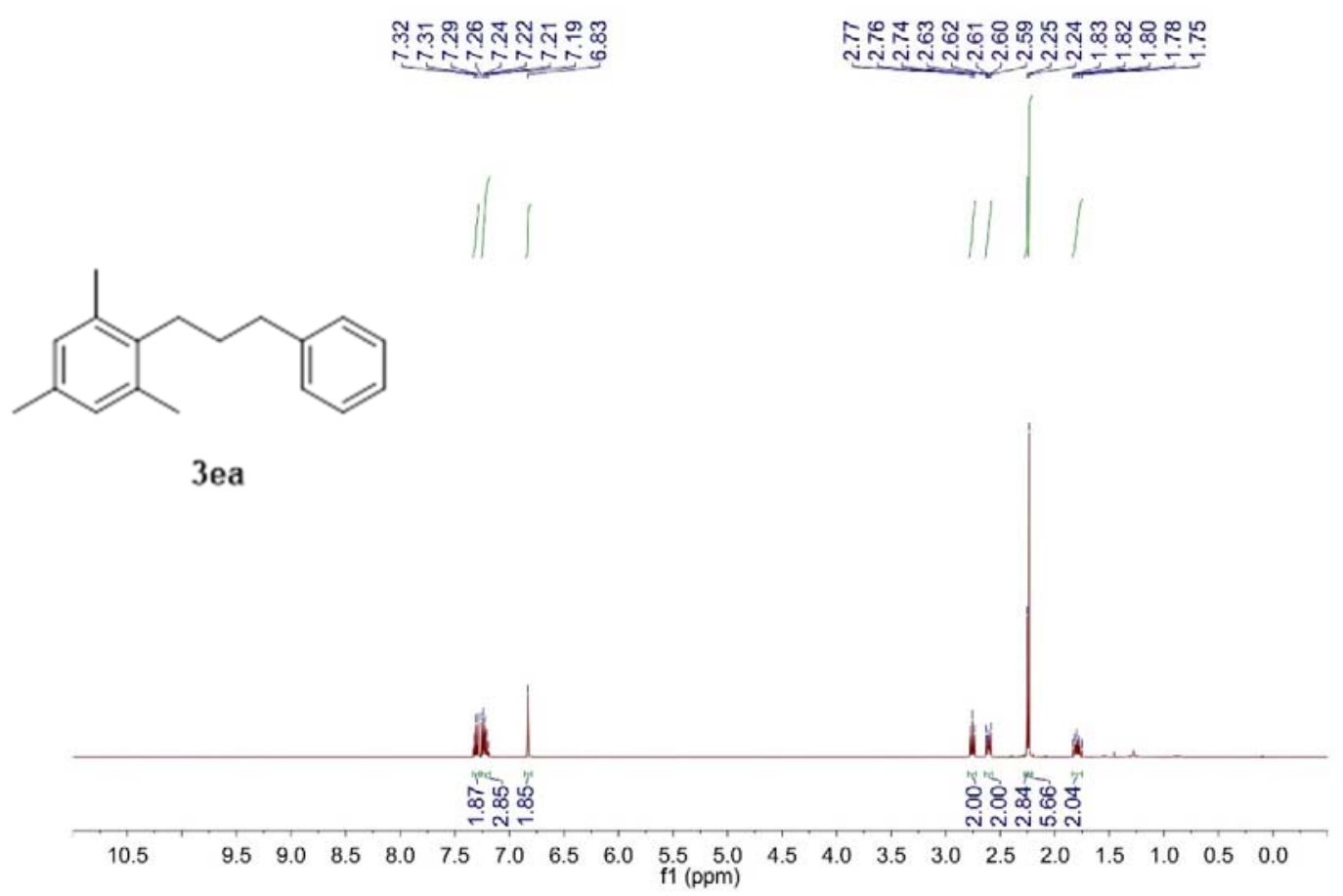

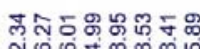

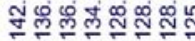

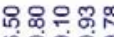

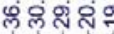

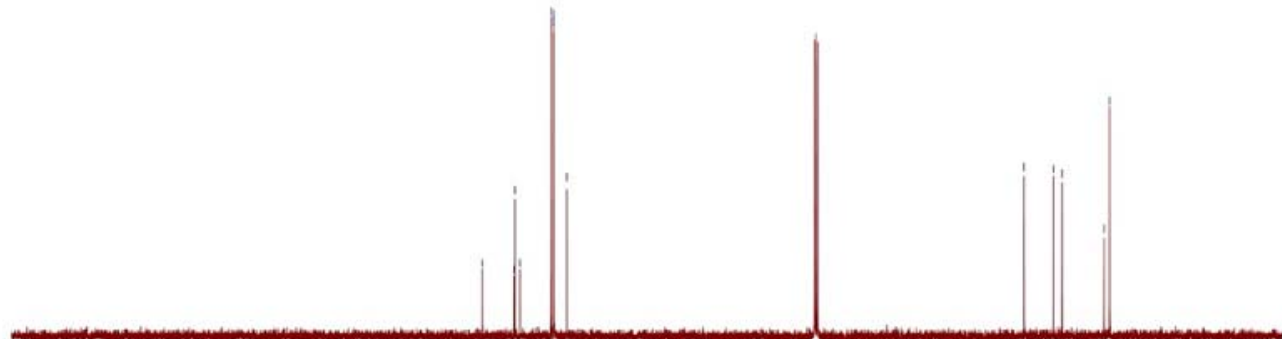

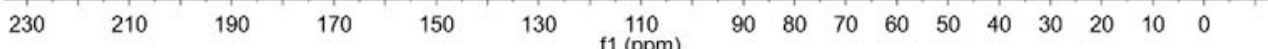

${ }^{1} \mathrm{H}$ NMR $(400 \mathrm{MHz})$ and ${ }^{13} \mathrm{C}\left\{{ }^{1} \mathrm{H}\right\}$ NMR $(101 \mathrm{MHz})$ spectra of 3ea (rt, $\left.\mathrm{CDCl}_{3}\right)$. 


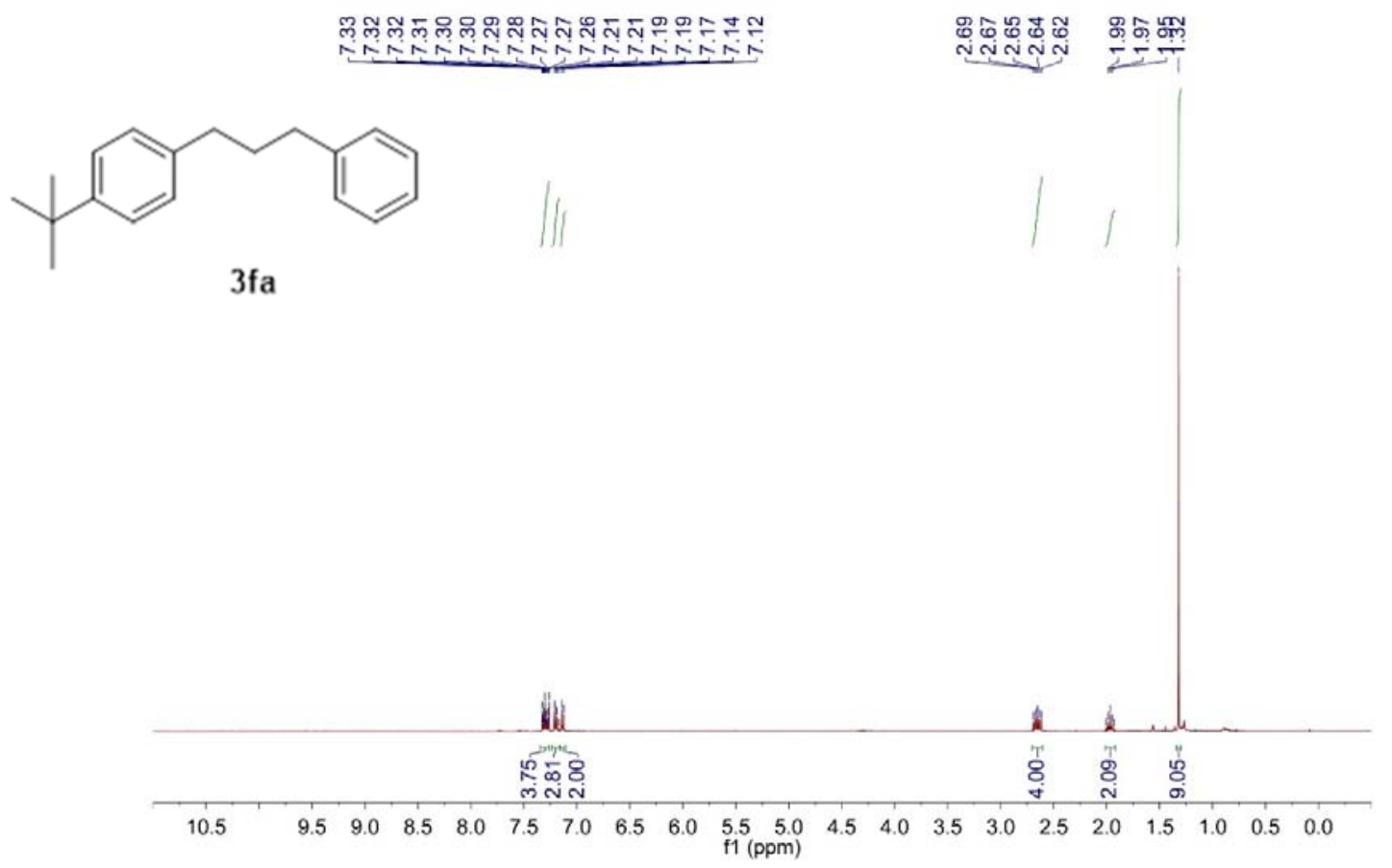

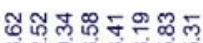

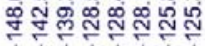

ํํㅇำ

फ्लिल्लिल

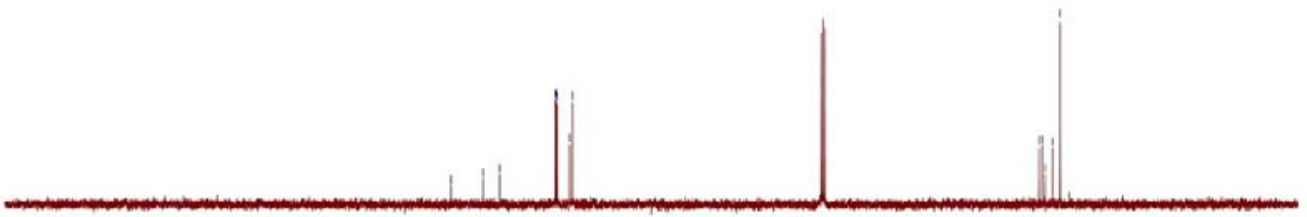

23

$210 \quad 190$

$190 \quad 150 \quad 130 \quad 110$

$\begin{array}{llllllllll}90 & 80 & 70 & 60 & 50 & 40 & 30 & 20 & 10 & 0\end{array}$

${ }^{1} \mathrm{H}$ NMR (400 MHz) and ${ }^{13} \mathrm{C}\left\{{ }^{1} \mathrm{H}\right\}$ NMR (101 MHz) spectra of $\mathbf{3 f a}\left(\mathrm{rt}, \mathrm{CDCl}_{3}\right)$. 


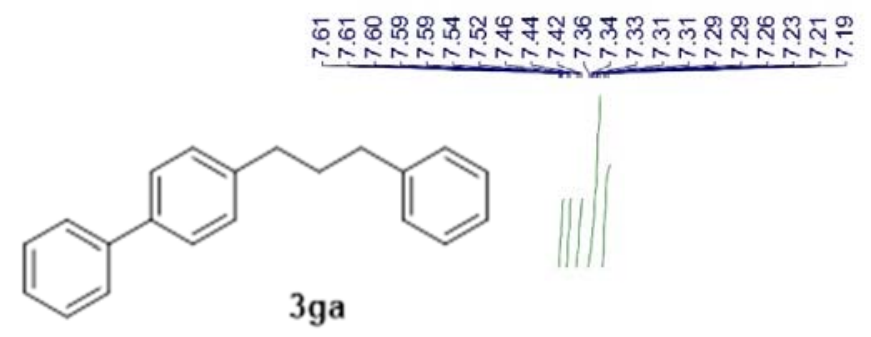

RNER :

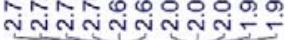
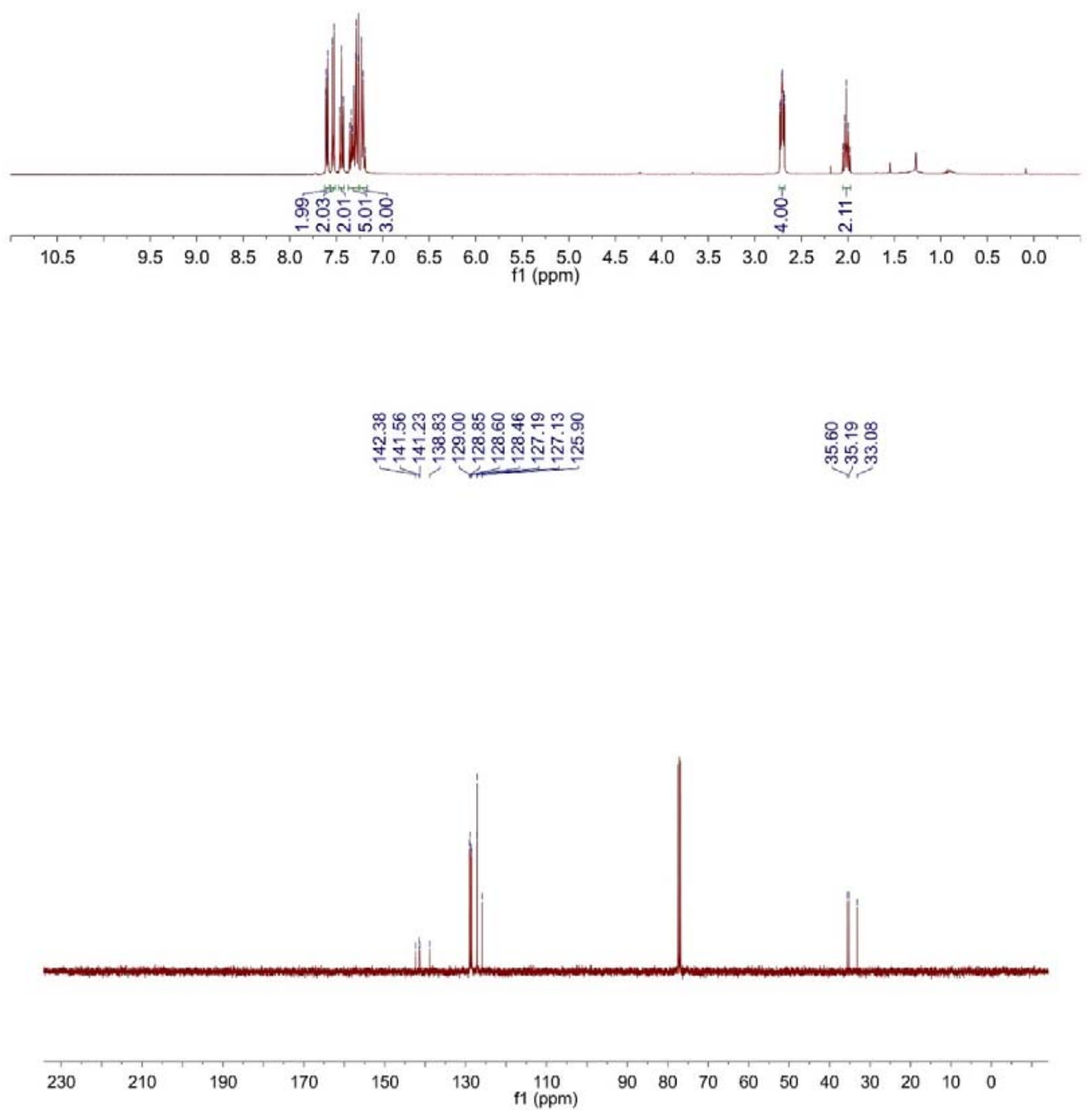

${ }^{1} \mathrm{H}$ NMR (400 MHz) and ${ }^{13} \mathrm{C}\left\{{ }^{1} \mathrm{H}\right\}$ NMR (101 MHz) spectra of 3ga (rt, $\left.\mathrm{CDCl}_{3}\right)$. 


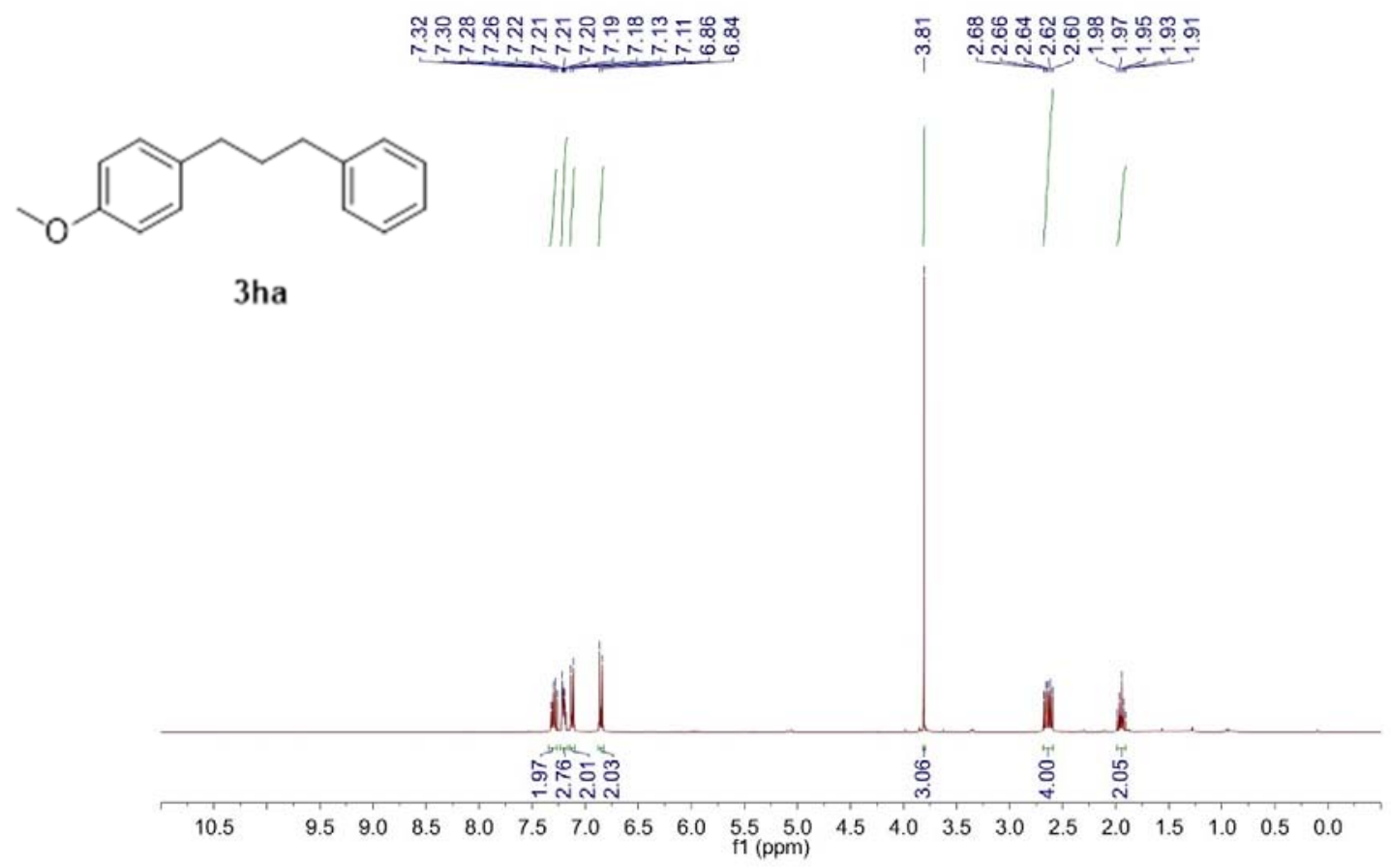

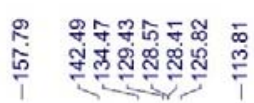

के क्षिळ

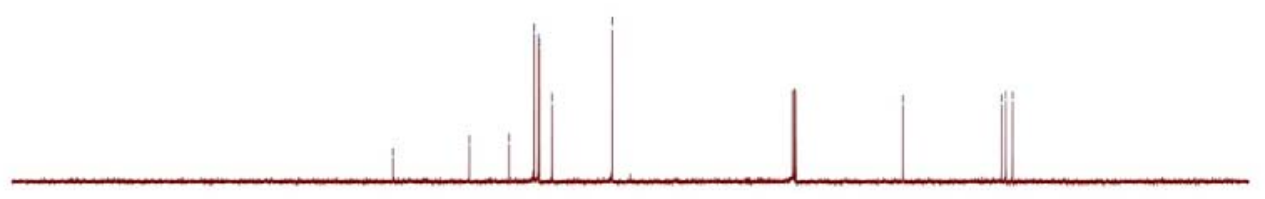

$\begin{array}{lllllllllllllllll}230 & 210 & 190 & 170 & 150 & 130 & \begin{array}{c}110 \\ \mathrm{f} 1(\mathrm{ppm})\end{array} & 90 & 80 & 70 & 60 & 50 & 40 & 30 & 20 & 10 & 0\end{array}$

${ }^{1} \mathrm{H}$ NMR $(400 \mathrm{MHz})$ and ${ }^{13} \mathrm{C}\left\{{ }^{1} \mathrm{H}\right\}$ NMR (101 MHz) spectra of 3ha $\left(\mathrm{rt}, \mathrm{CDCl}_{3}\right)$. 


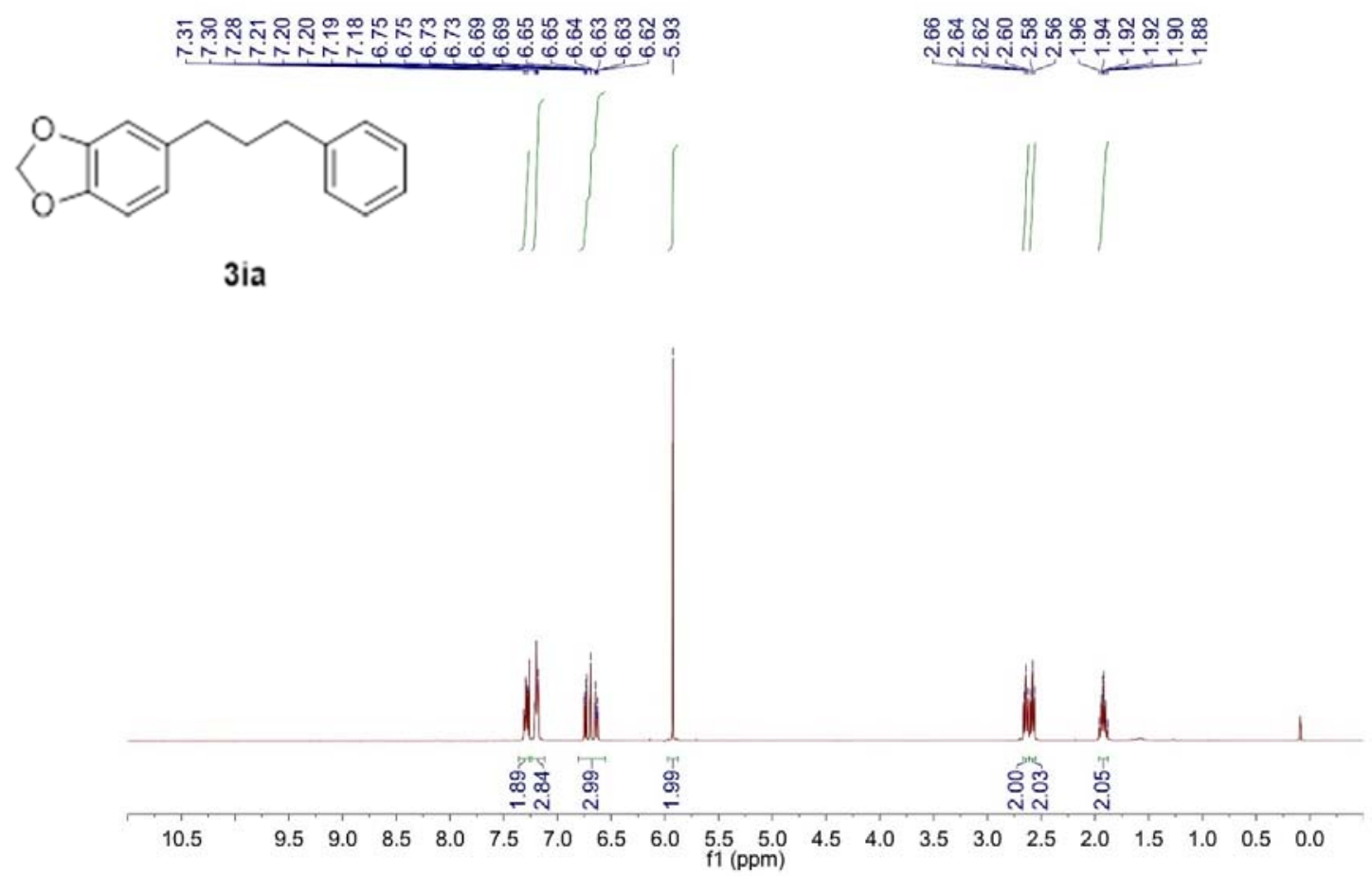

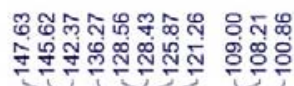

于तกิ

이용

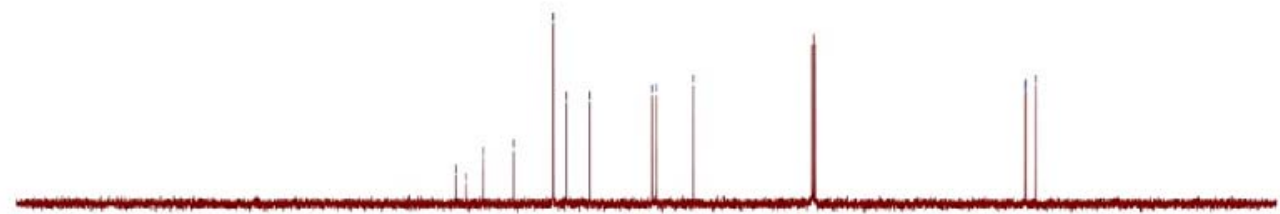

230

210

190

$130 \quad 110$

$\begin{array}{llllllllll}90 & 80 & 70 & 60 & 50 & 40 & 30 & 20 & 10 & 0\end{array}$

${ }^{1} \mathrm{H}$ NMR $(400 \mathrm{MHz})$ and ${ }^{13} \mathrm{C}\left\{{ }^{1} \mathrm{H}\right\}$ NMR $(101 \mathrm{MHz})$ spectrum of 3ia (rt, $\left.\mathrm{CDCl}_{3}\right)$. 

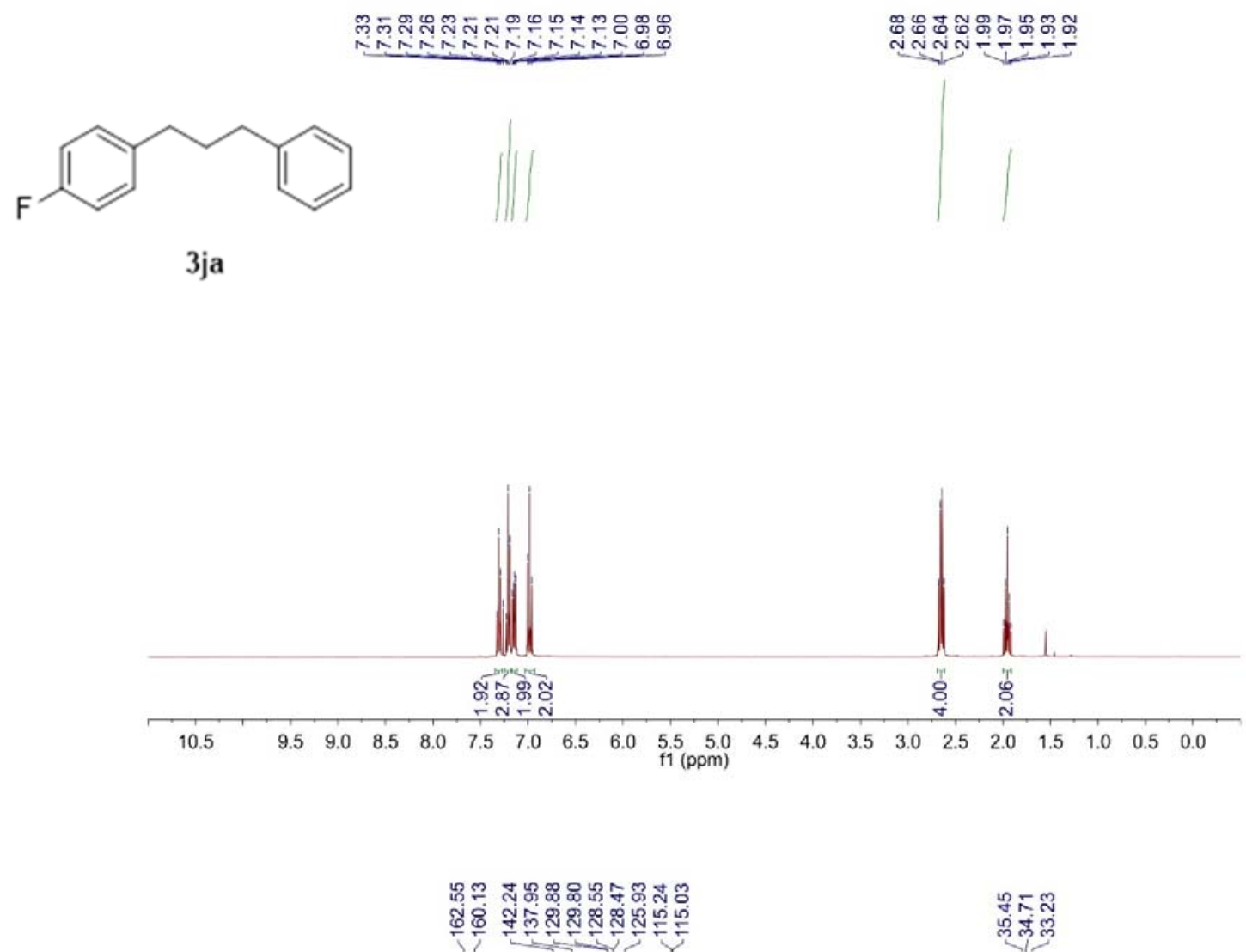

भก๊

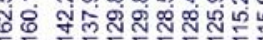

हैंलेल

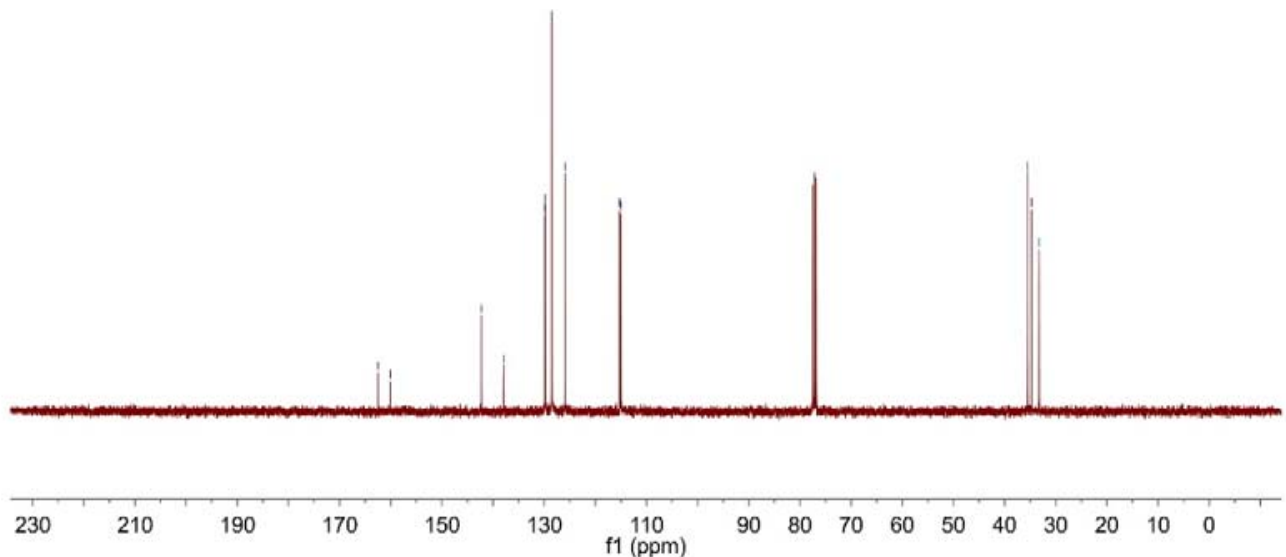




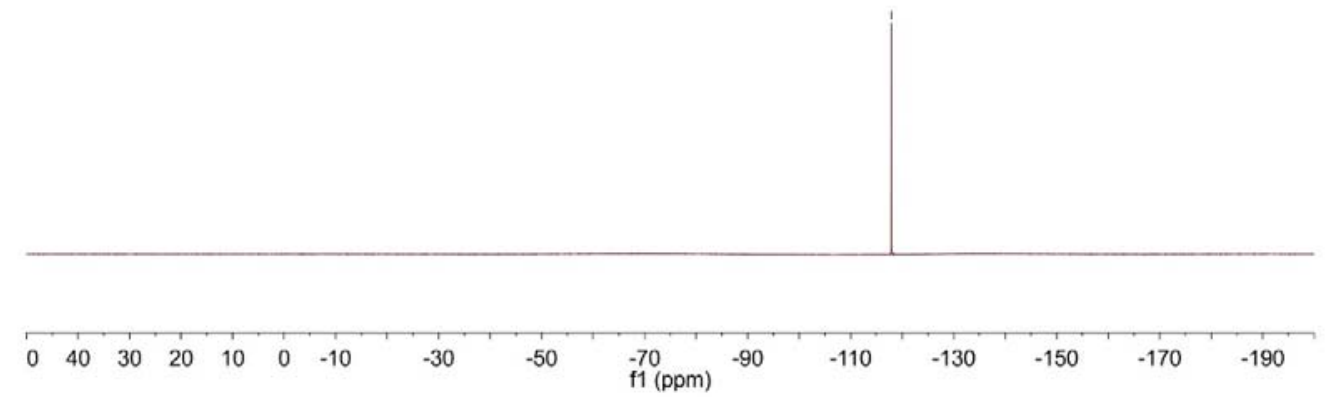

${ }^{1} \mathrm{H}$ NMR (400 MHz), ${ }^{13} \mathrm{C}\left\{{ }^{1} \mathrm{H}\right\}$ NMR (101 MHz), and ${ }^{19} \mathrm{~F}\left\{{ }^{1} \mathrm{H}\right\}$ NMR (376 MHz) spectra of $3 \mathbf{j a}\left(\mathrm{rt}, \mathrm{CDCl}_{3}\right)$. 


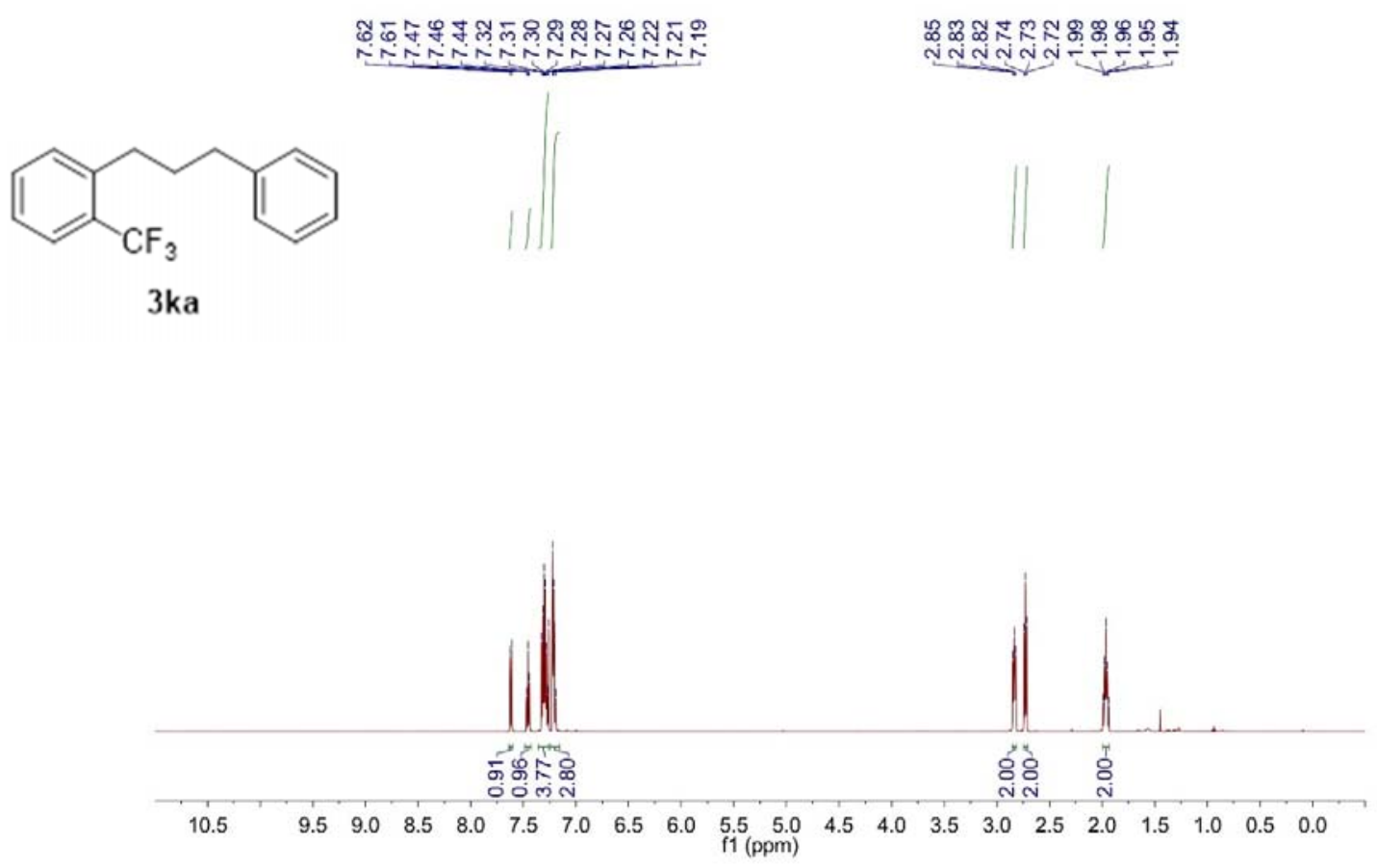

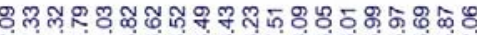

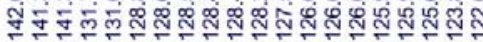

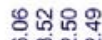

म्लूल्लूल

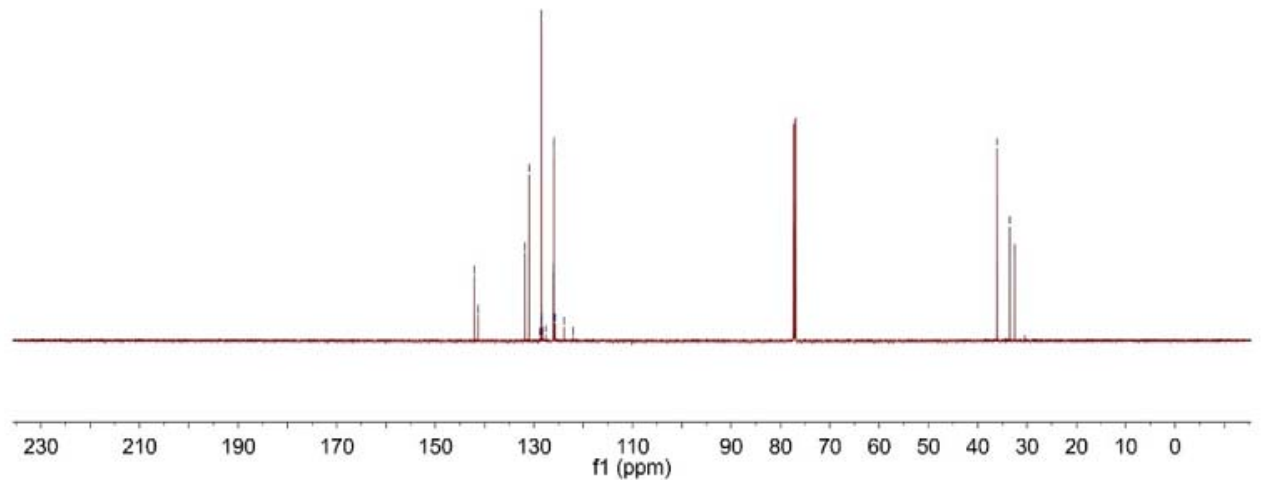




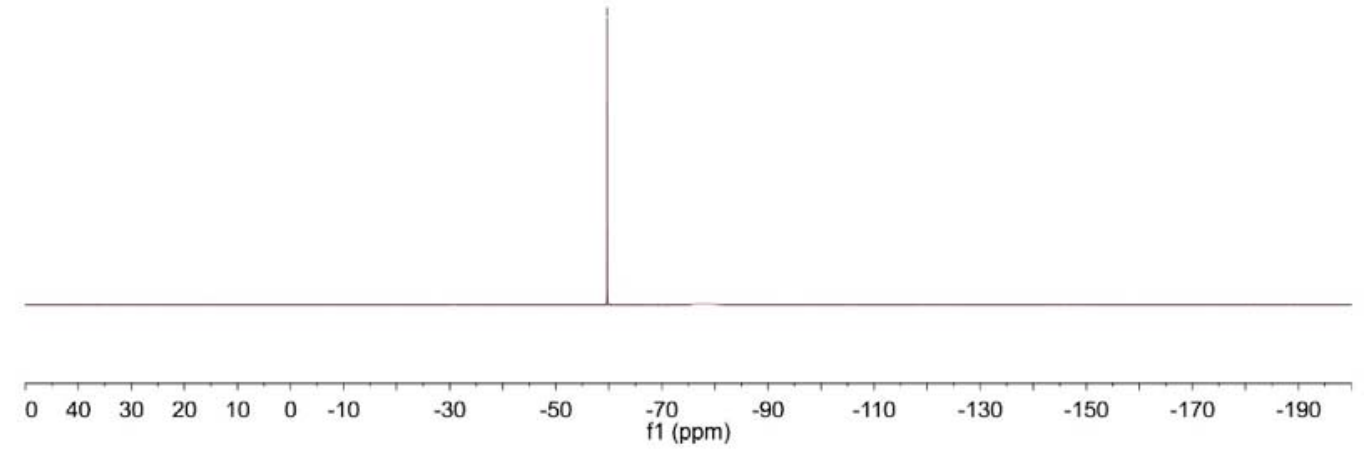

${ }^{1} \mathrm{H}$ NMR $(600 \mathrm{MHz}),{ }^{13} \mathrm{C}\left\{{ }^{1} \mathrm{H}\right\}$ NMR $(151 \mathrm{MHz})$, and ${ }^{19} \mathrm{~F}\left\{{ }^{1} \mathrm{H}\right\}$ NMR $(282 \mathrm{MHz})$ spectra of 3ka $\left(\mathrm{rt}, \mathrm{CDCl}_{3}\right)$. 


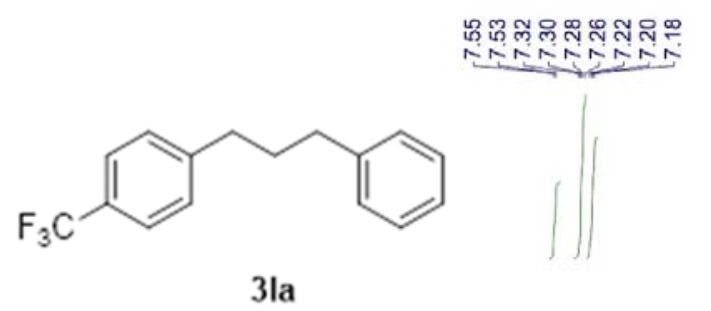

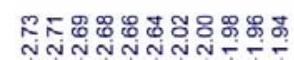
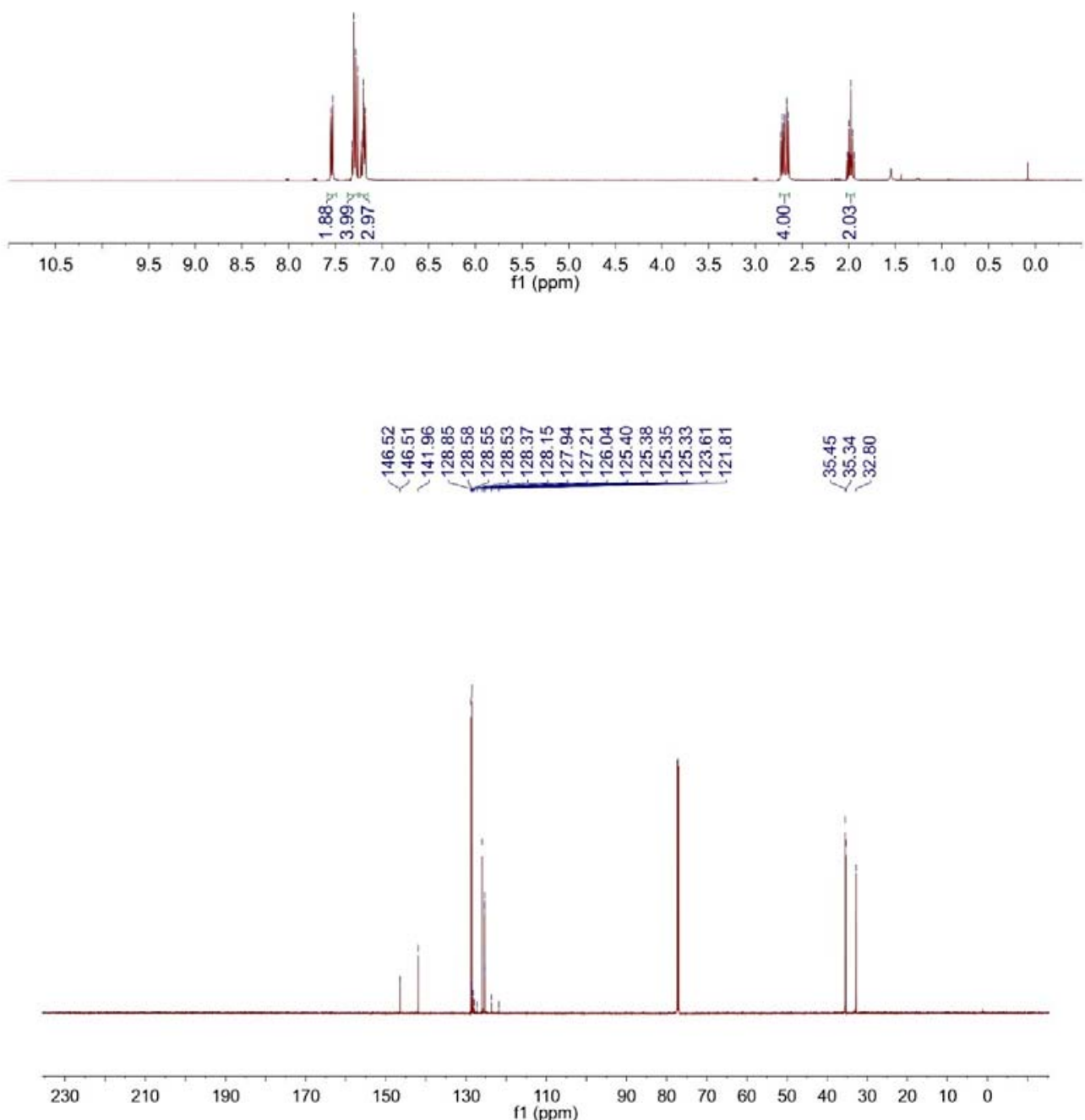


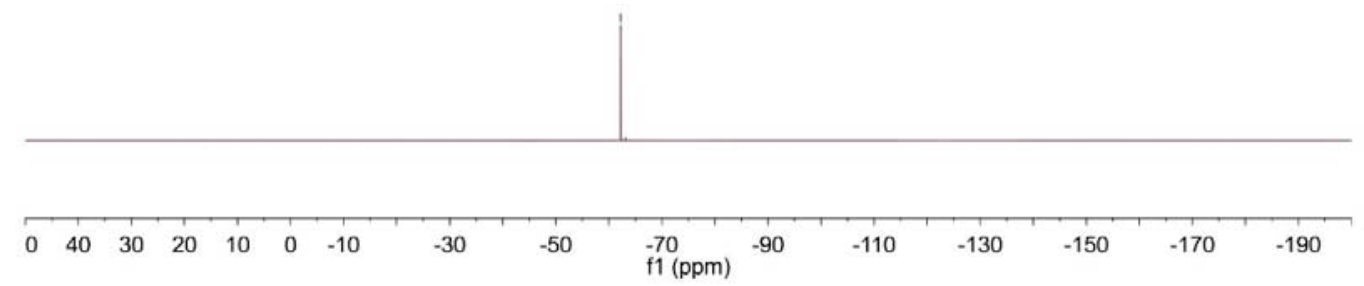

${ }^{1} \mathrm{H}$ NMR (400 MHz), ${ }^{13} \mathrm{C}\left\{{ }^{1} \mathrm{H}\right\}$ NMR (151 MHz), and ${ }^{19} \mathrm{~F}\left\{{ }^{1} \mathrm{H}\right\}$ NMR $(376 \mathrm{MHz})$ spectra of 3la $\left(\mathrm{rt}, \mathrm{CDCl}_{3}\right)$. 

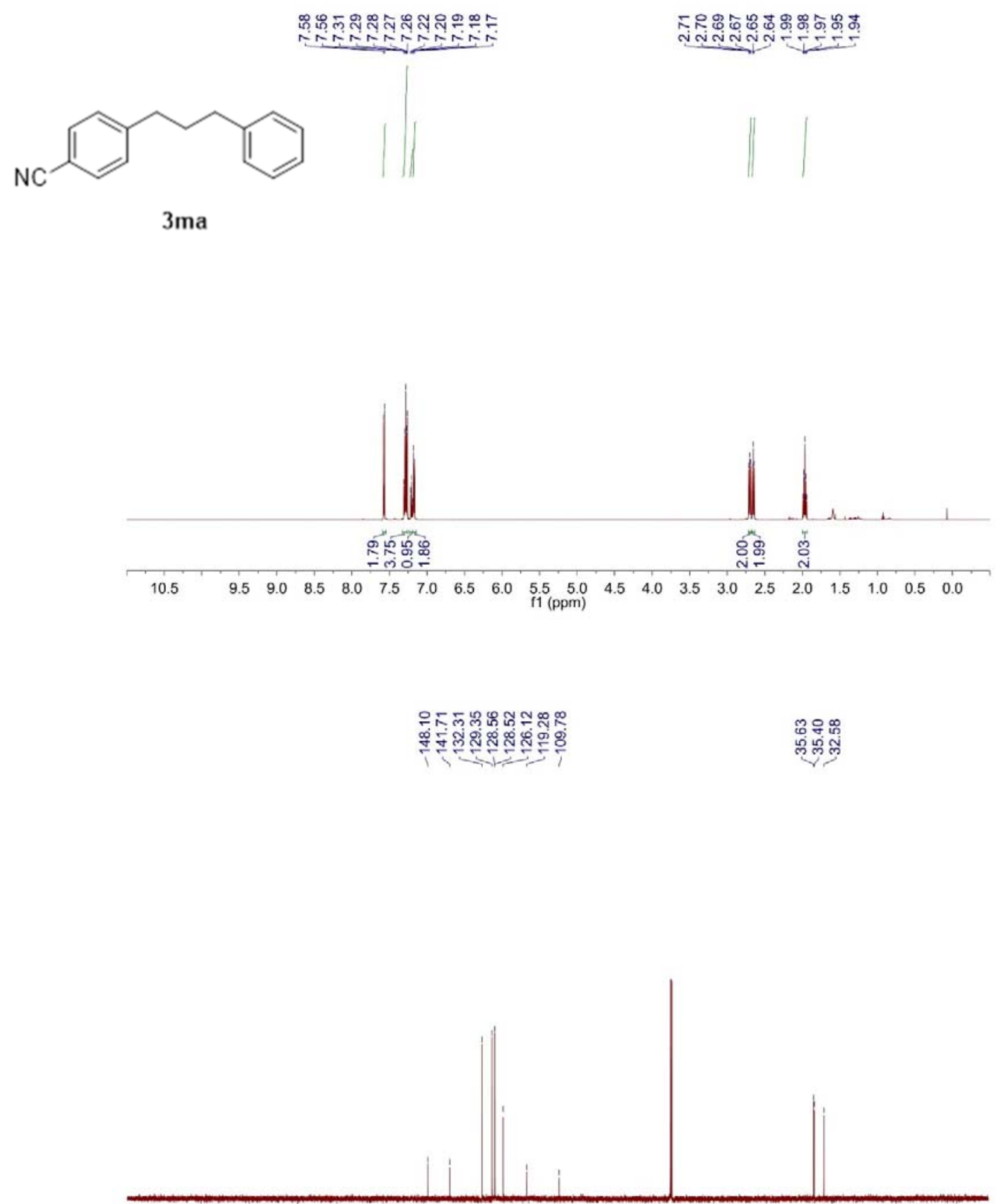

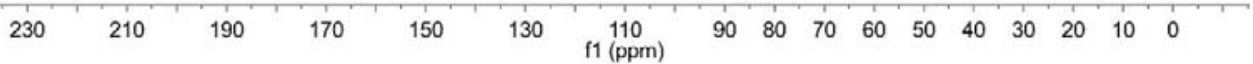

${ }^{1} \mathrm{H}$ NMR (600 MHz) and ${ }^{13} \mathrm{C}\left\{{ }^{1} \mathrm{H}\right\}$ NMR (151 MHz) spectra of 3ma (rt, $\left.\mathrm{CDCl}_{3}\right)$. 

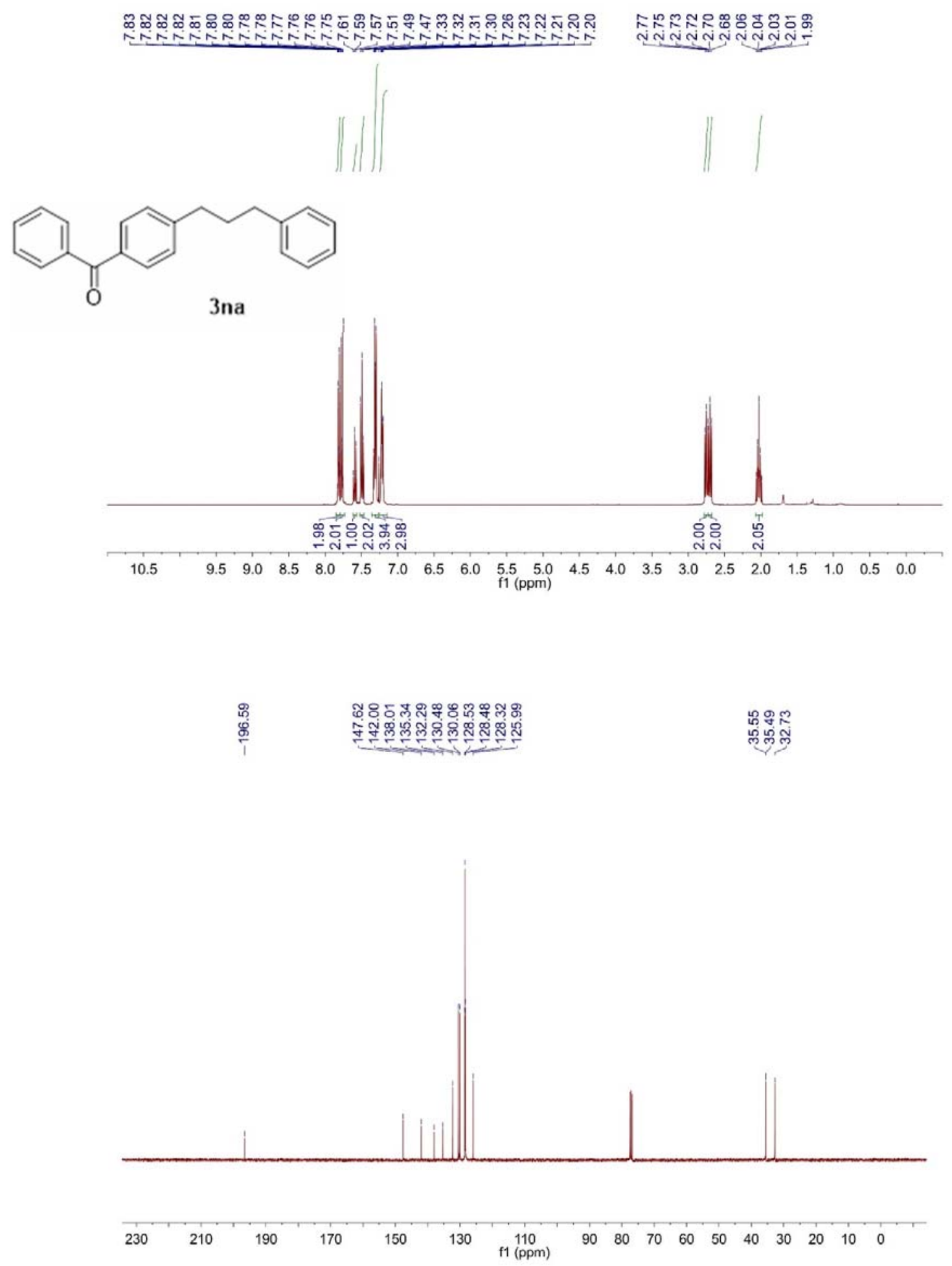

${ }^{1} \mathrm{H}$ NMR (400 MHz) and ${ }^{13} \mathrm{C}\left\{{ }^{1} \mathrm{H}\right\}$ NMR (101 MHz) spectra of 3na (rt, $\left.\mathrm{CDCl}_{3}\right)$. 

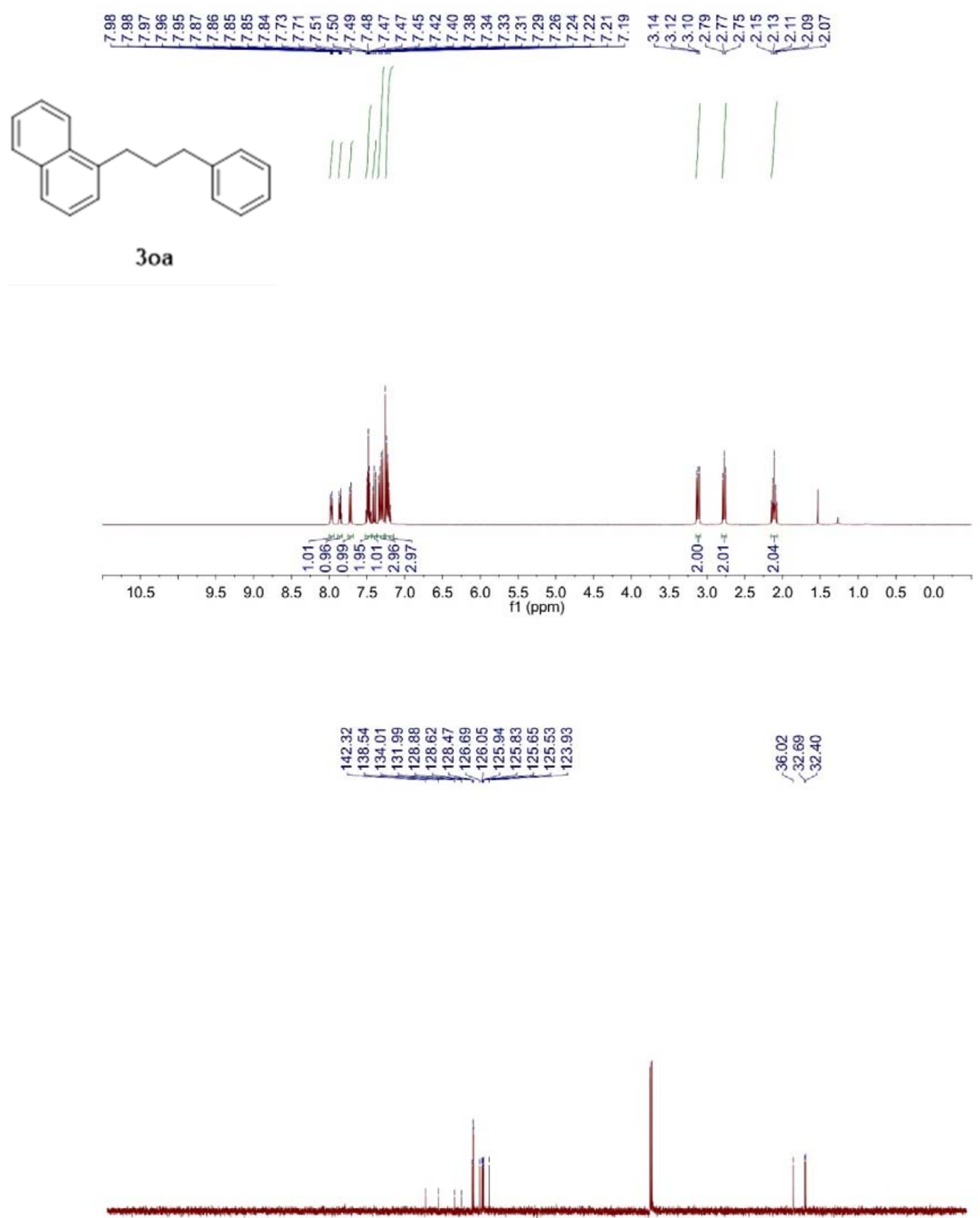

${ }^{1} \mathrm{H}$ NMR $(400 \mathrm{MHz})$ and ${ }^{13} \mathrm{C}\left\{{ }^{1} \mathrm{H}\right\}$ NMR $(101 \mathrm{MHz})$ spectra of 3oa (rt, $\left.\mathrm{CDCl}_{3}\right)$. 


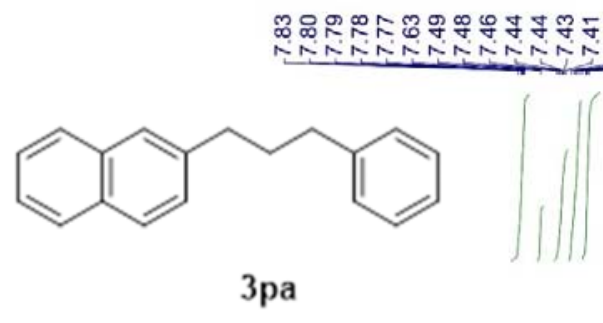

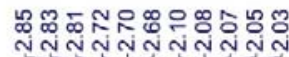
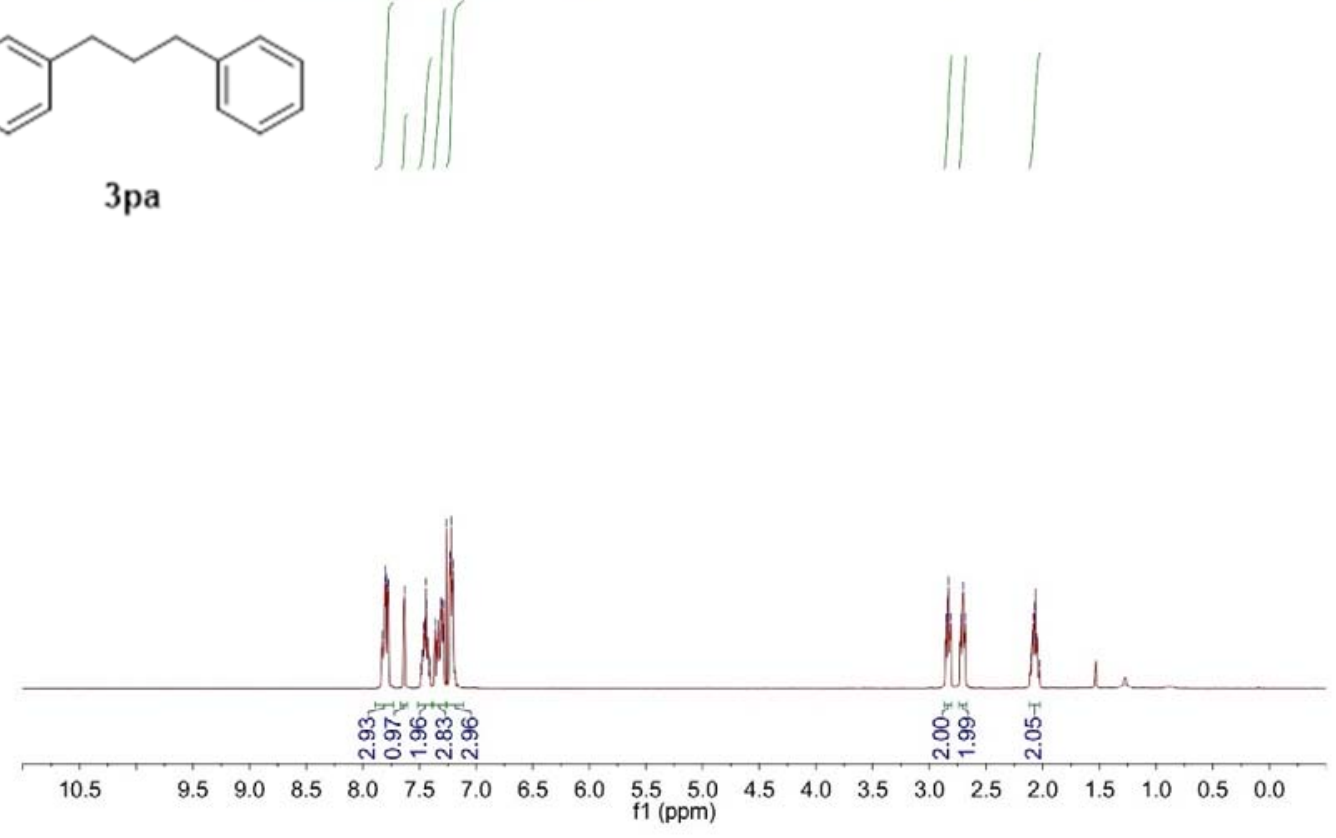

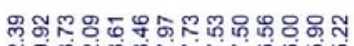

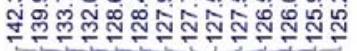

다요

लेंलुलु

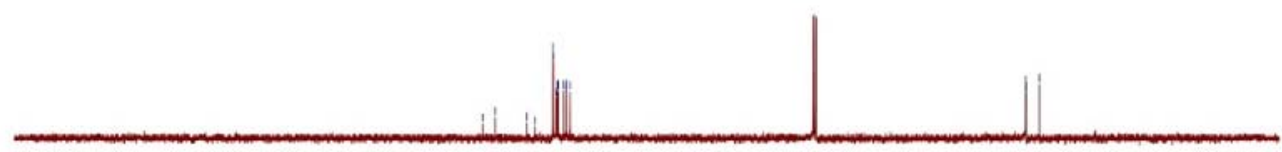

230 $210 \quad 190 \quad 170 \quad 150 \quad 130 \quad 110$

$\begin{array}{llllllllll}90 & 80 & 70 & 60 & 50 & 40 & 30 & 20 & 10 & 0\end{array}$

${ }^{1} \mathrm{H}$ NMR (400 MHz) and ${ }^{13} \mathrm{C}\left\{{ }^{1} \mathrm{H}\right\}$ NMR (101 MHz) spectra of 3pa (rt, $\left.\mathrm{CDCl}_{3}\right)$. 


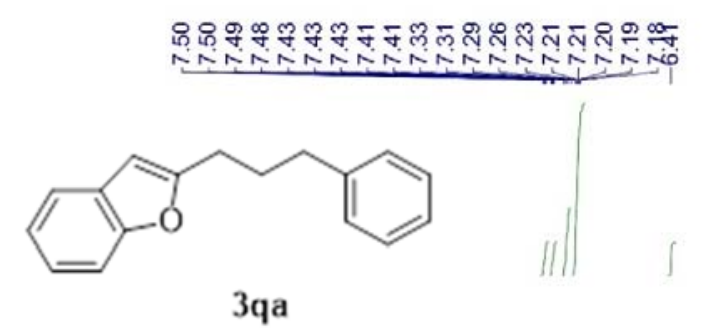

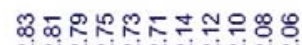

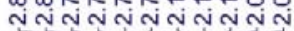
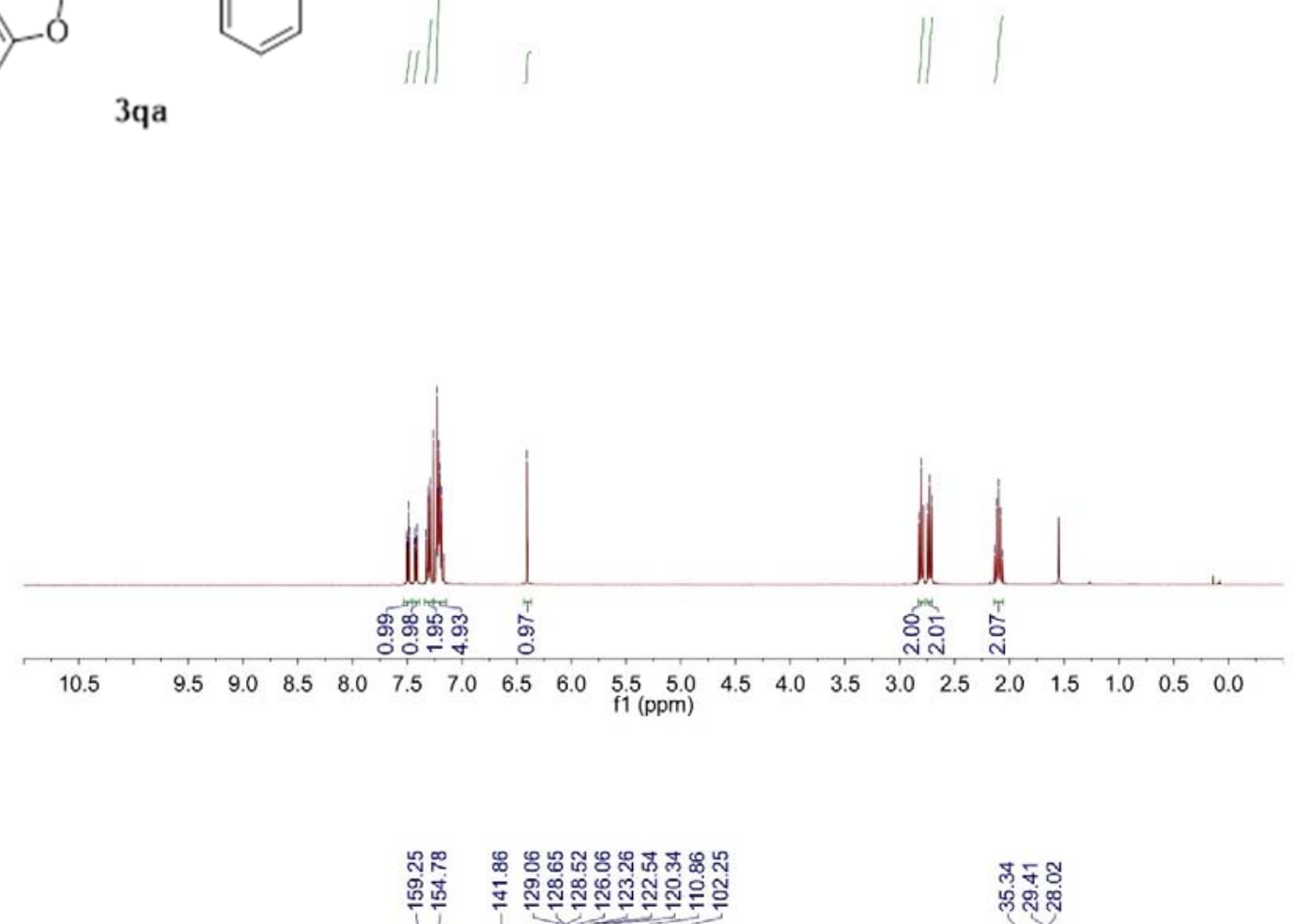

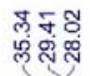

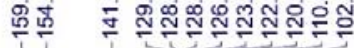

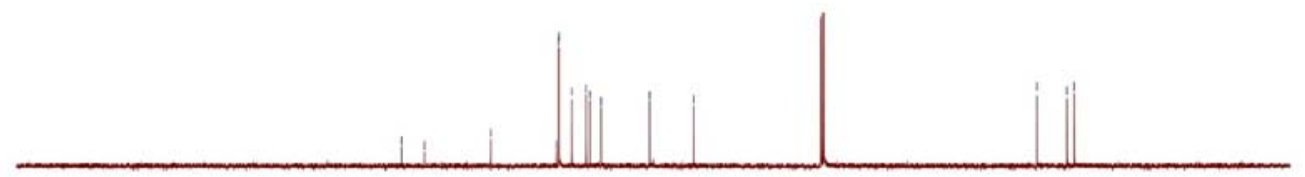

230

$210 \quad 190 \quad 170$

$50 \quad 130 \quad 110$

$\begin{array}{llllllllll}90 & 80 & 70 & 60 & 50 & 40 & 30 & 20 & 10 & 0\end{array}$

${ }^{1} \mathrm{H}$ NMR (400 MHz) and ${ }^{13} \mathrm{C}\left\{{ }^{1} \mathrm{H}\right\}$ NMR (101 MHz) spectra of 3qa (rt, $\left.\mathrm{CDCl}_{3}\right)$. 


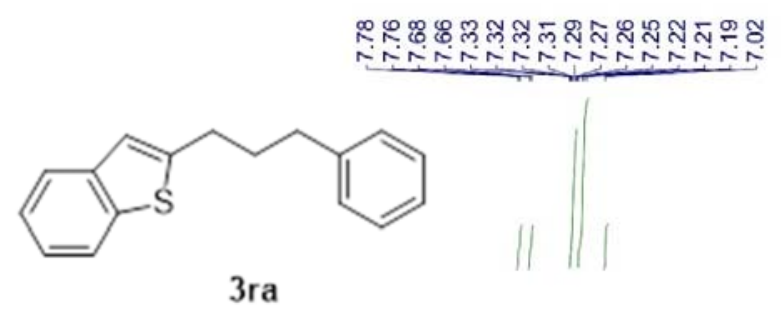

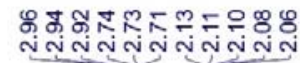

3 ra

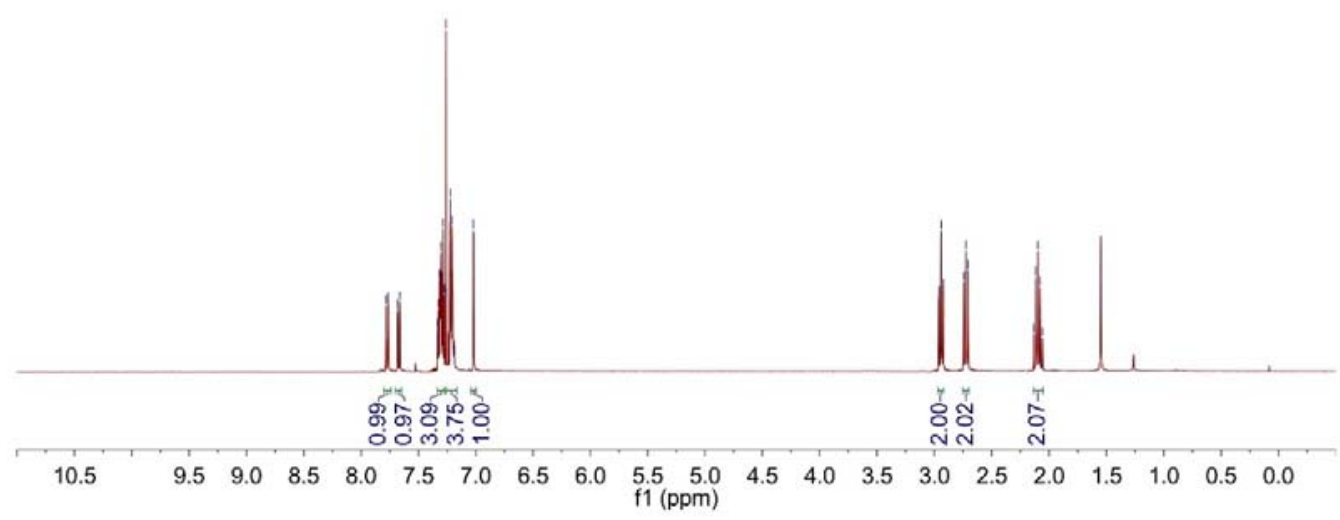

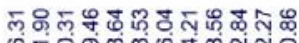

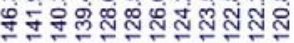

뉴용

फल्ले

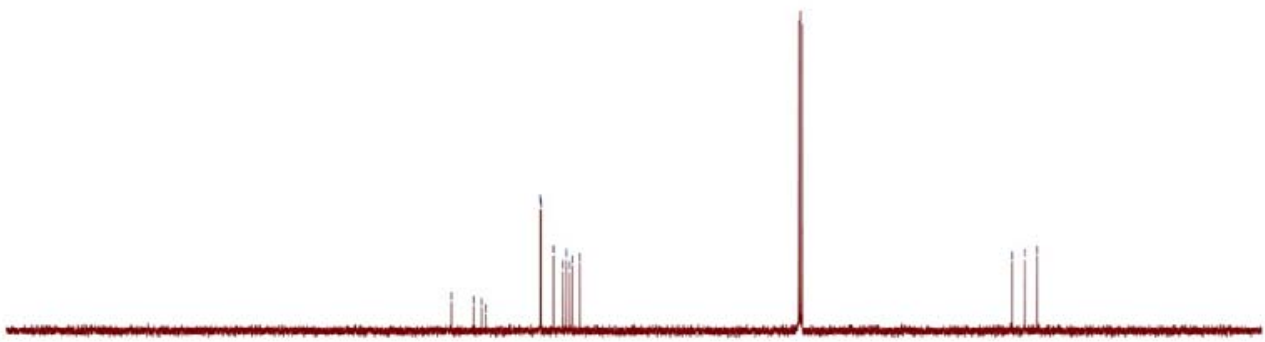

230

$190 \quad 170 \quad 150 \quad 130 \quad 110$

$\begin{array}{llllllllll}90 & 80 & 70 & 60 & 50 & 40 & 30 & 20 & 10 & 0\end{array}$

${ }^{1} \mathrm{H}$ NMR (400 MHz) and ${ }^{13} \mathrm{C}\left\{{ }^{1} \mathrm{H}\right\}$ NMR (101 MHz) spectra of 3ra (rt, $\left.\mathrm{CDCl}_{3}\right)$. 

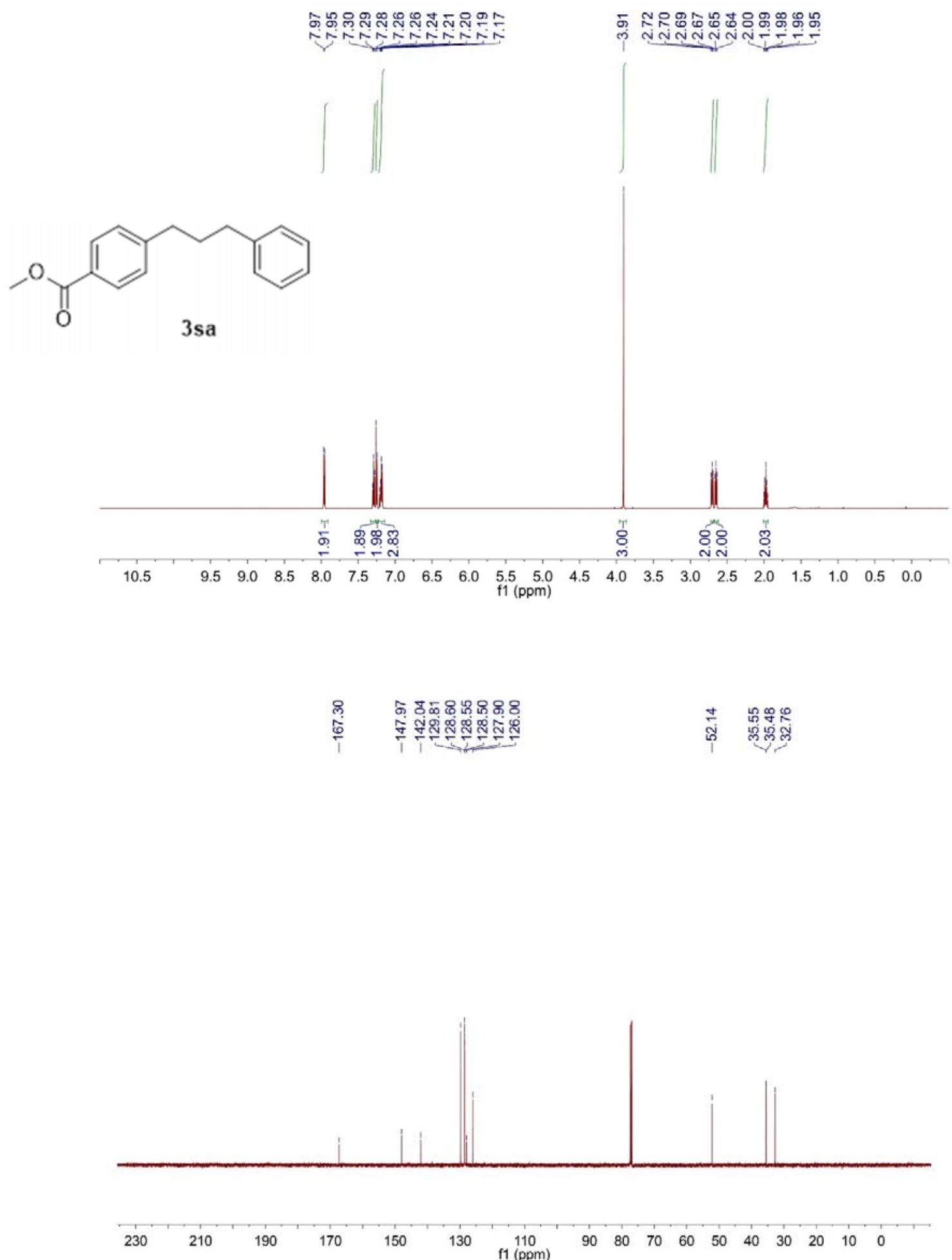

${ }^{1} \mathrm{H}$ NMR $(600 \mathrm{MHz})$ and ${ }^{13} \mathrm{C}\left\{{ }^{1} \mathrm{H}\right\}$ NMR $(151 \mathrm{MHz})$ spectra of 3sa $\left(\mathrm{rt}, \mathrm{CDCl}_{3}\right)$. 

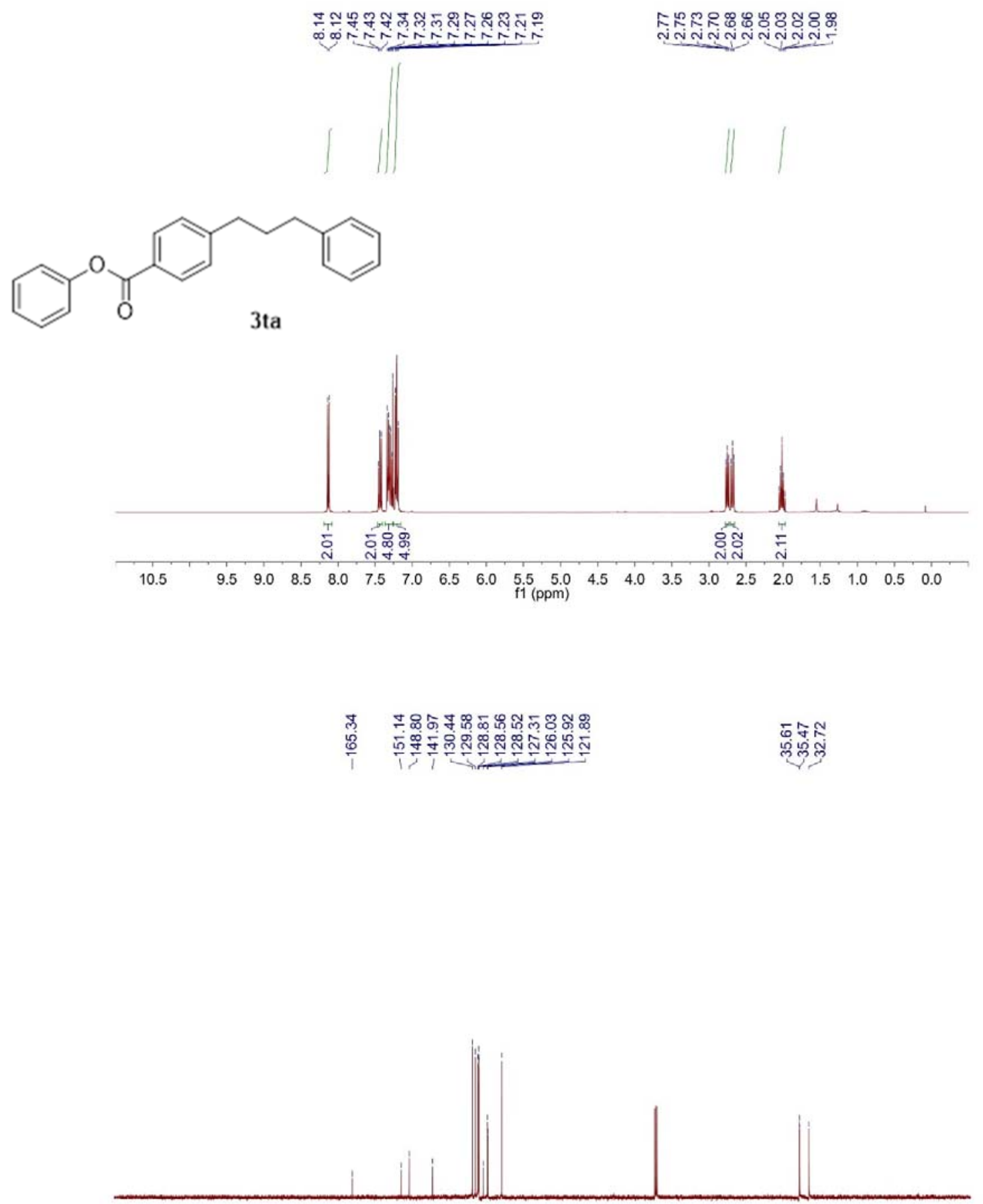

230

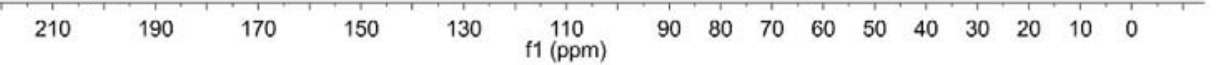

${ }^{1} \mathrm{H}$ NMR $(400 \mathrm{MHz})$ and ${ }^{13} \mathrm{C}\left\{{ }^{1} \mathrm{H}\right\}$ NMR $(101 \mathrm{MHz})$ spectra of 3ta $\left(\mathrm{rt}, \mathrm{CDCl}_{3}\right)$. 


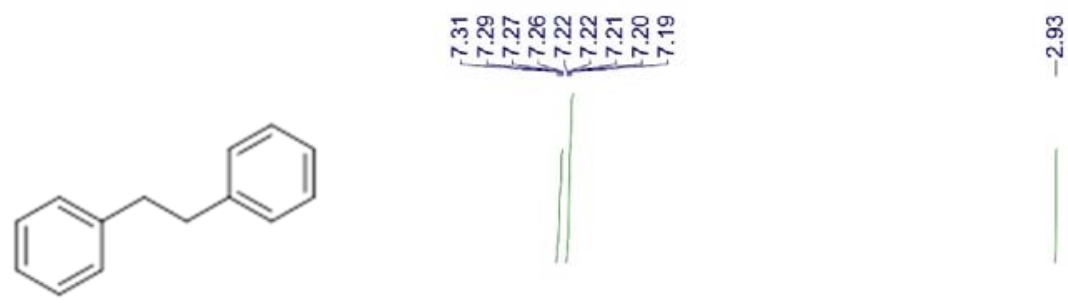

$3 a b$

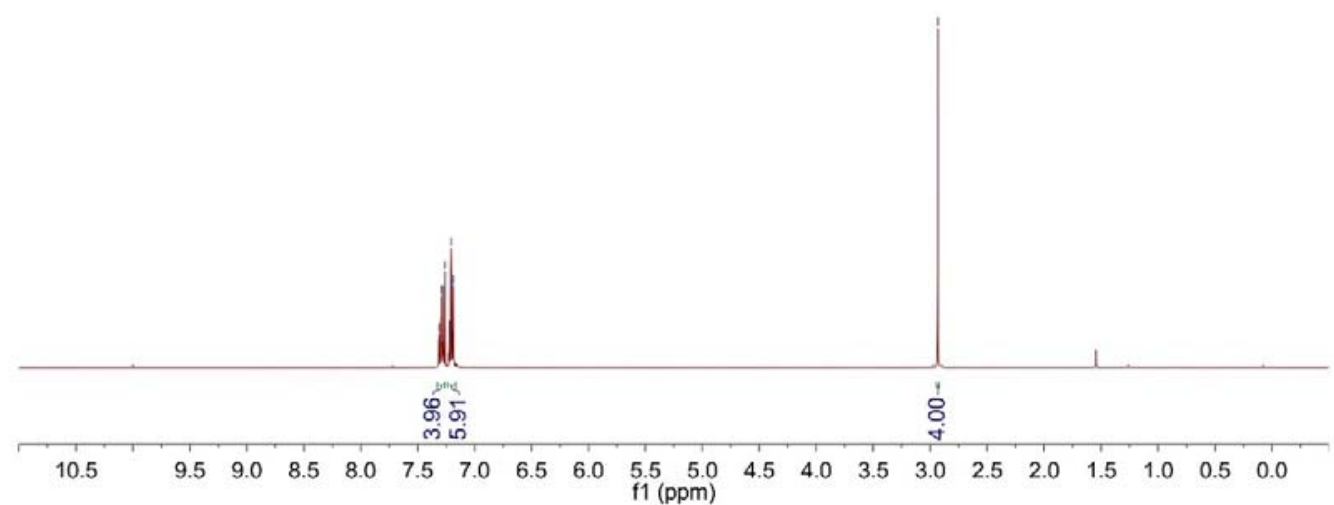

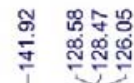

$\underset{\infty}{\stackrel{0}{\infty}}$

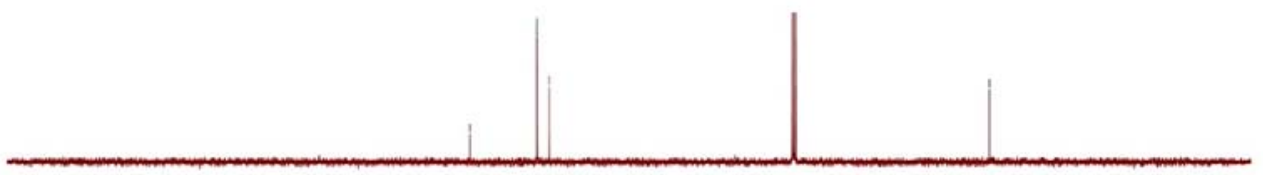

230

$210 \quad 190$

170 110
$\mathrm{f} 1(\mathrm{ppm})$

$\begin{array}{llllllllll}90 & 80 & 70 & 60 & 50 & 40 & 30 & 20 & 10 & 0\end{array}$

${ }^{1} \mathrm{H}$ NMR (400 MHz) and ${ }^{13} \mathrm{C}\left\{{ }^{1} \mathrm{H}\right\}$ NMR (101 MHz) spectra of 3ab (rt, $\left.\mathrm{CDCl}_{3}\right)$. 


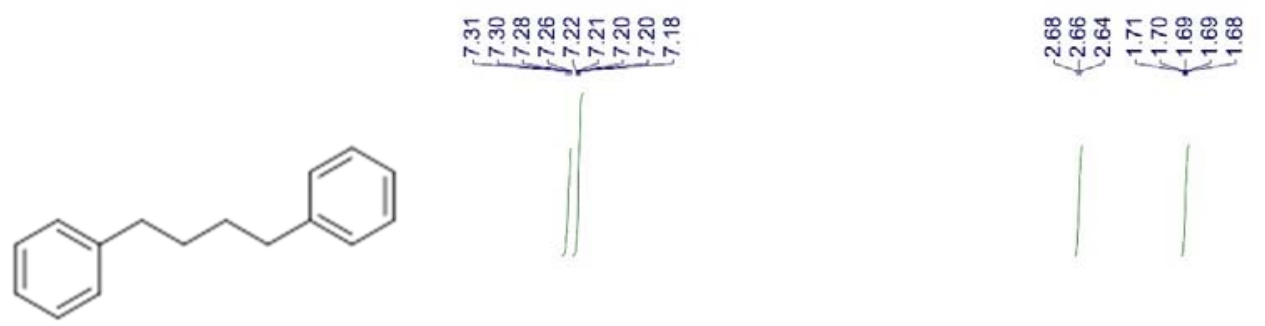

3ac

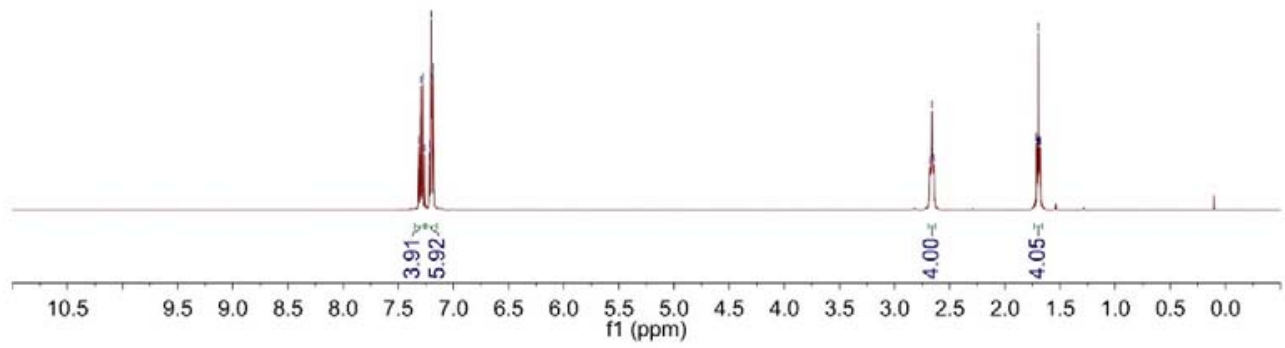

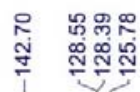

迎

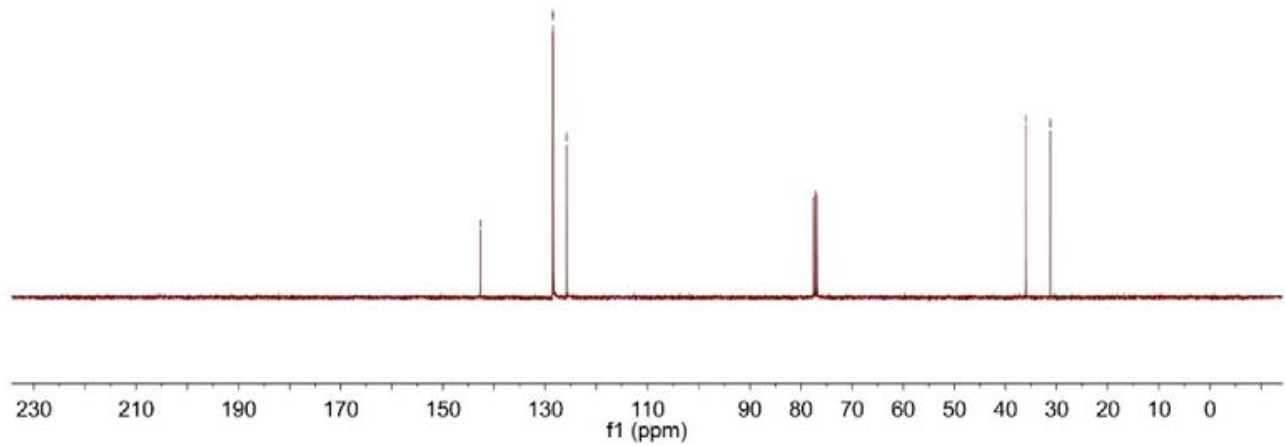

${ }^{1} \mathrm{H}$ NMR (400 MHz) and ${ }^{13} \mathrm{C}\left\{{ }^{1} \mathrm{H}\right\}$ NMR (101 MHz) spectra of 3ac (rt, $\left.\mathrm{CDCl}_{3}\right)$. 


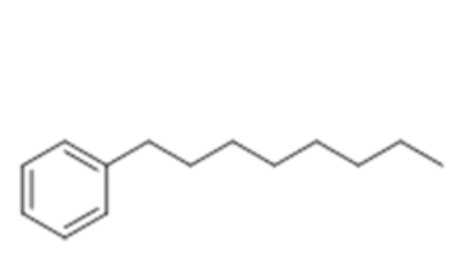

3ad

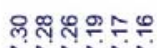

intisis

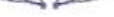

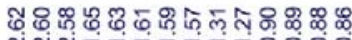

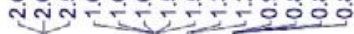
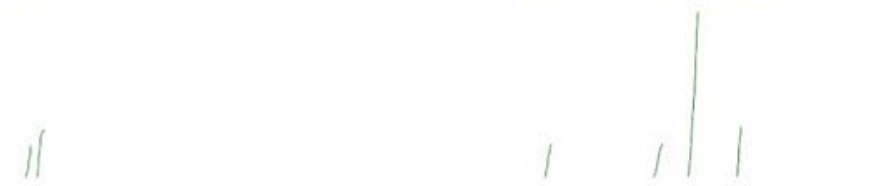

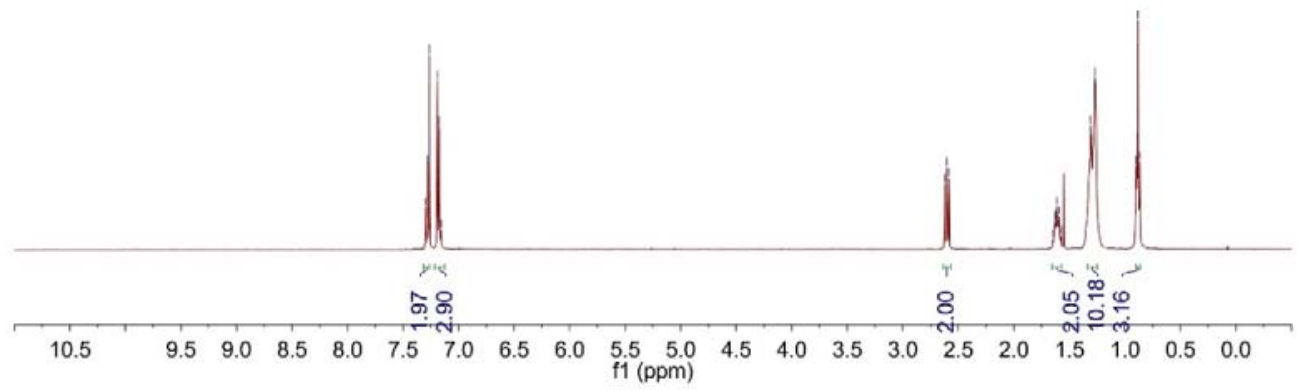

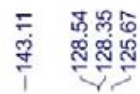

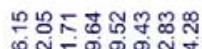

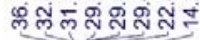

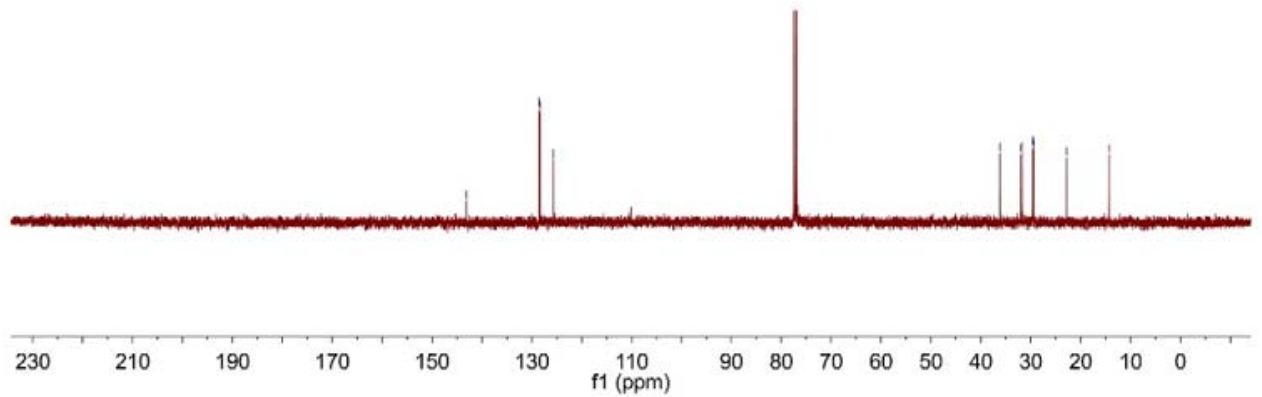

${ }^{1} \mathrm{H}$ NMR (400 MHz) and ${ }^{13} \mathrm{C}\left\{{ }^{1} \mathrm{H}\right\}$ NMR (101 MHz) spectra of 3ad (rt, $\left.\mathrm{CDCl}_{3}\right)$. 

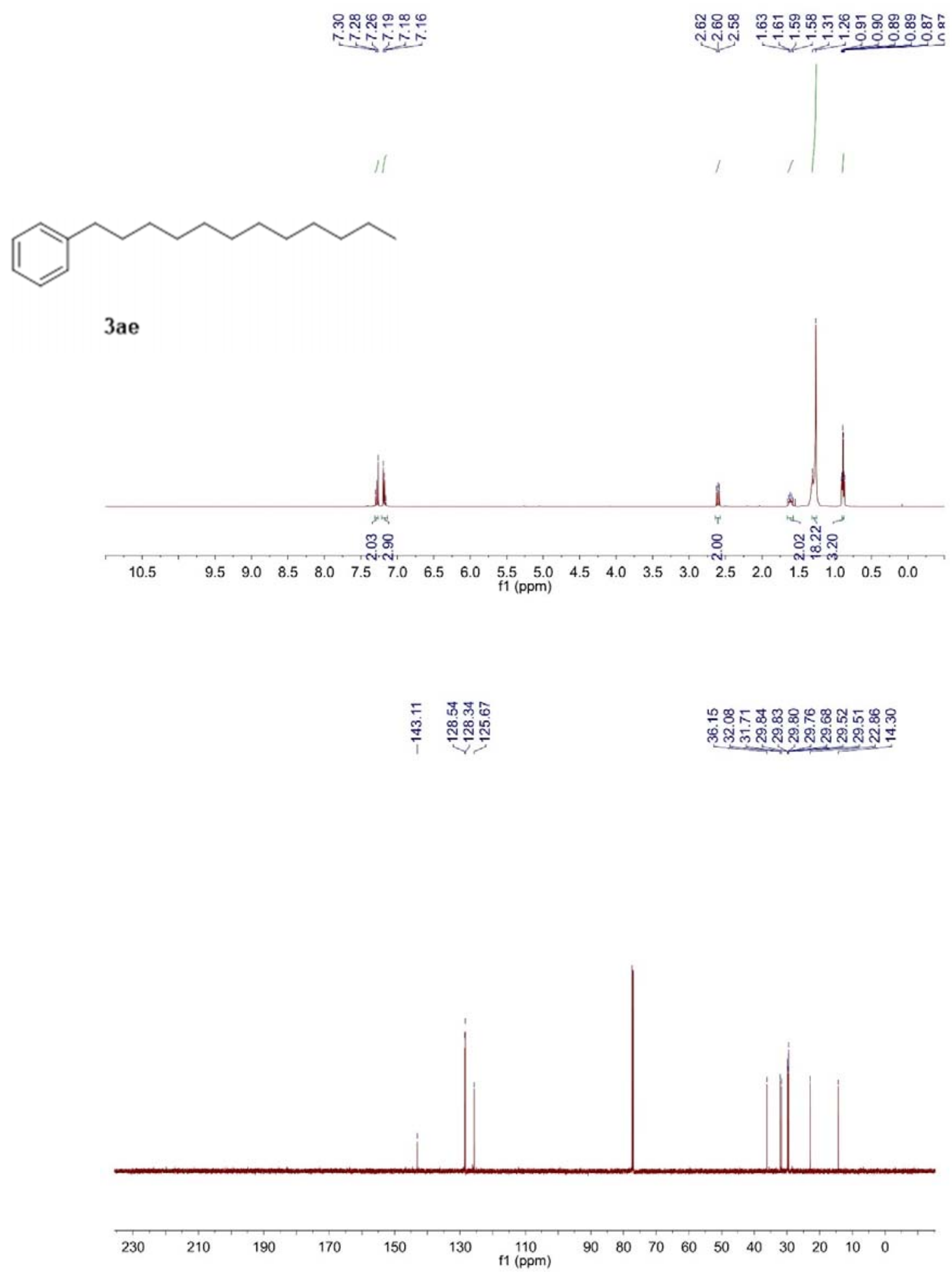

${ }^{1} \mathrm{H}$ NMR (400 MHz) and ${ }^{13} \mathrm{C}\left\{{ }^{1} \mathrm{H}\right\}$ NMR $(151 \mathrm{MHz})$ spectrum of 3ae (rt, $\left.\mathrm{CDCl}_{3}\right)$. 

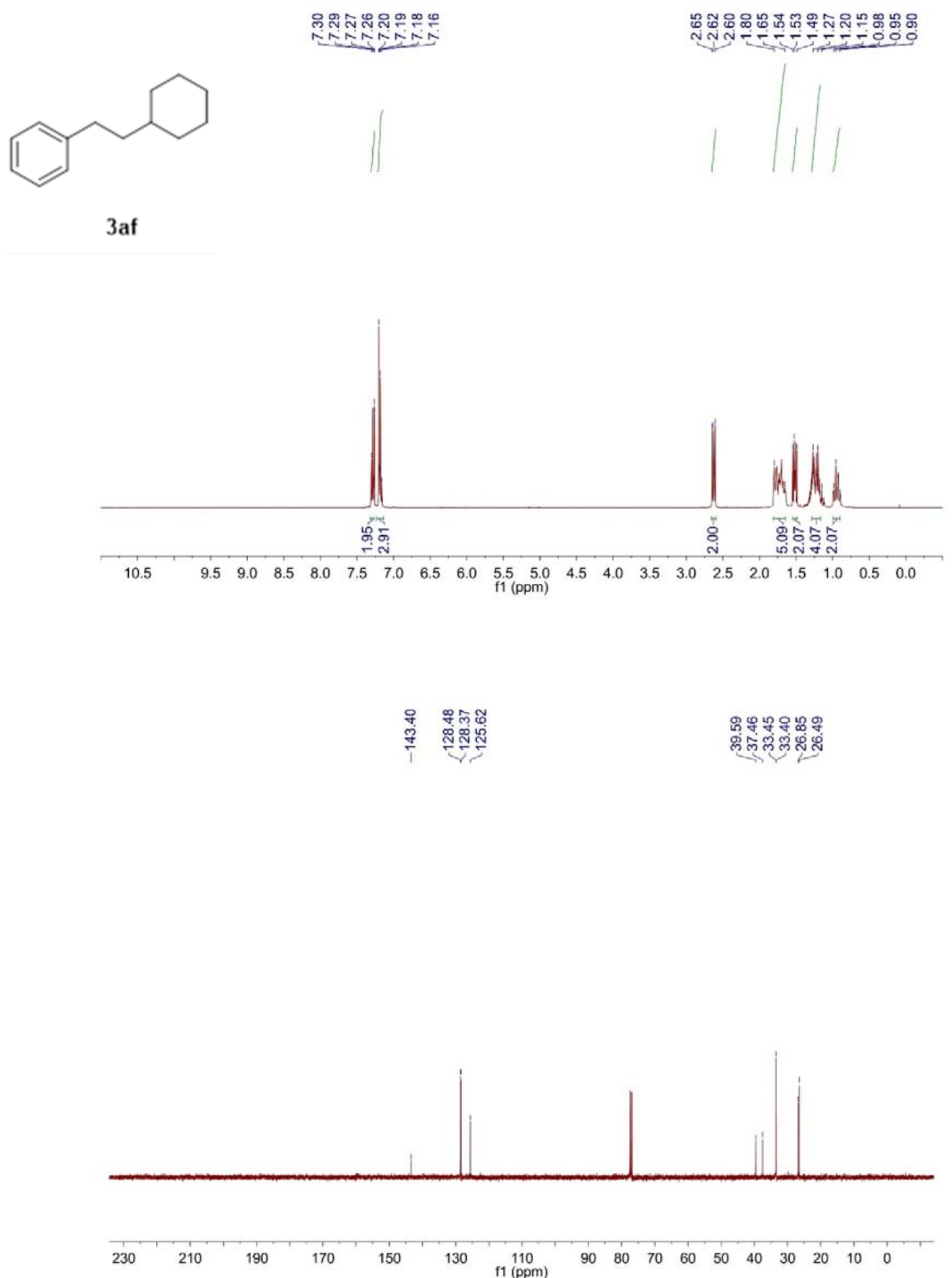

${ }^{1} \mathrm{H}$ NMR $(400 \mathrm{MHz})$ and ${ }^{13} \mathrm{C}\left\{{ }^{1} \mathrm{H}\right\}$ NMR $(101 \mathrm{MHz})$ spectrum of 3af $\left(\mathrm{rt}, \mathrm{CDCl}_{3}\right)$. 


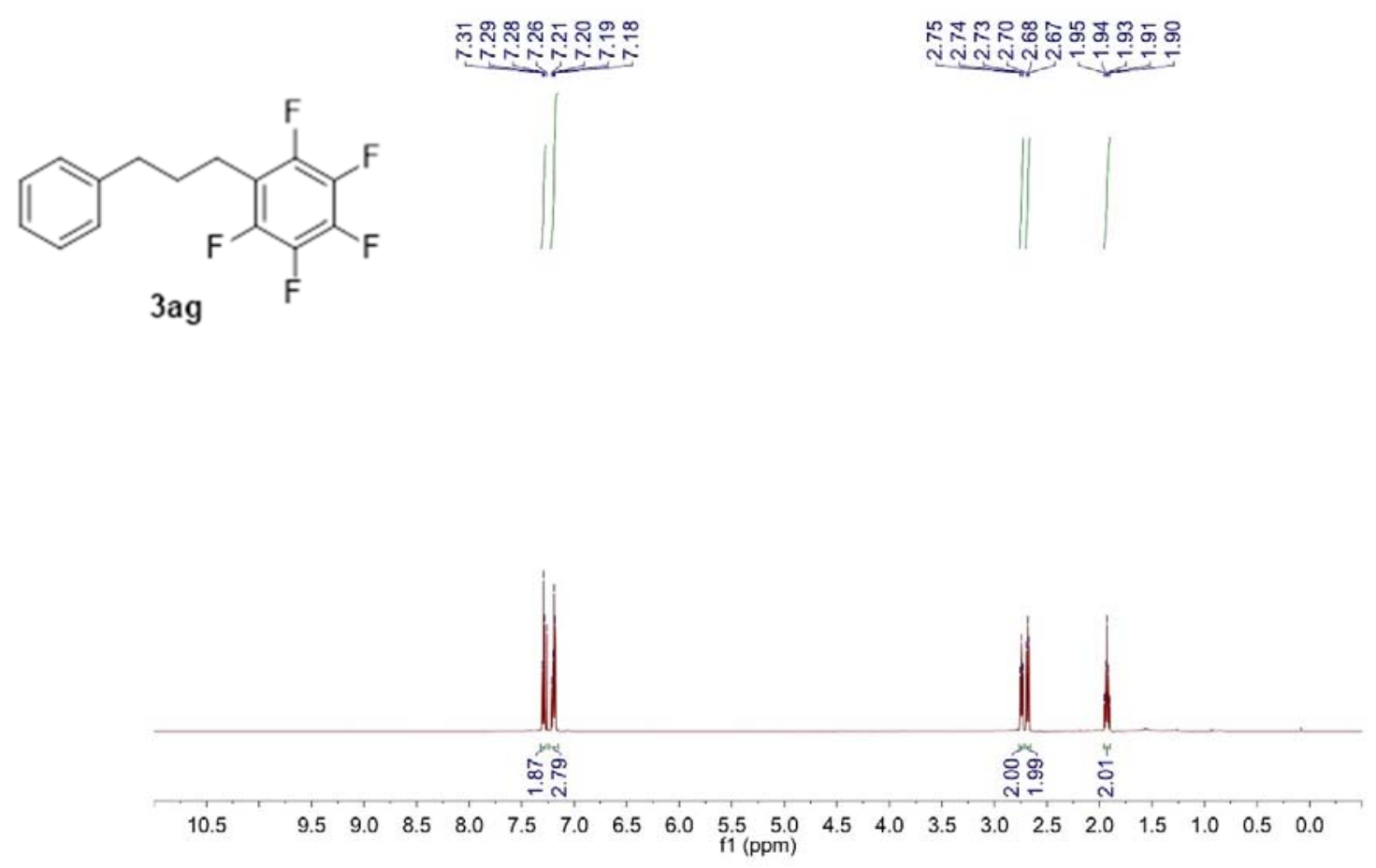

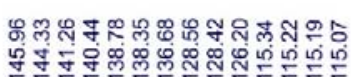

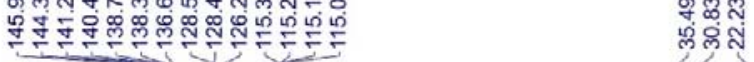

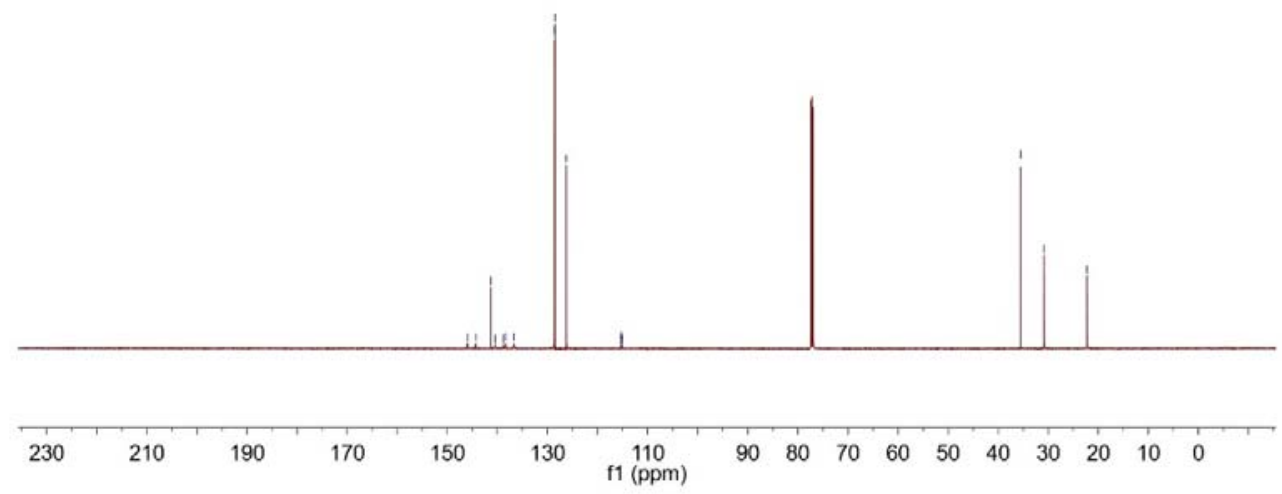


ㄷำกิร

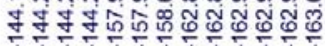

itifis

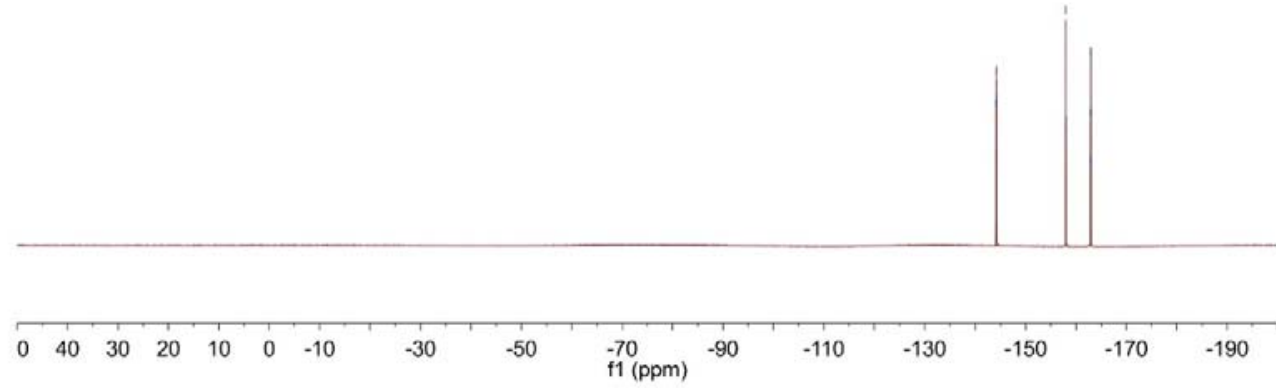

${ }^{1} \mathrm{H}$ NMR (600 MHz), ${ }^{13} \mathrm{C}\left\{{ }^{1} \mathrm{H}\right\}$ NMR (151 MHz), and ${ }^{19} \mathrm{~F}\left\{{ }^{1} \mathrm{H}\right\}$ NMR (376 MHz) spectra of $3 a g\left(r t, \mathrm{CDCl}_{3}\right)$. 

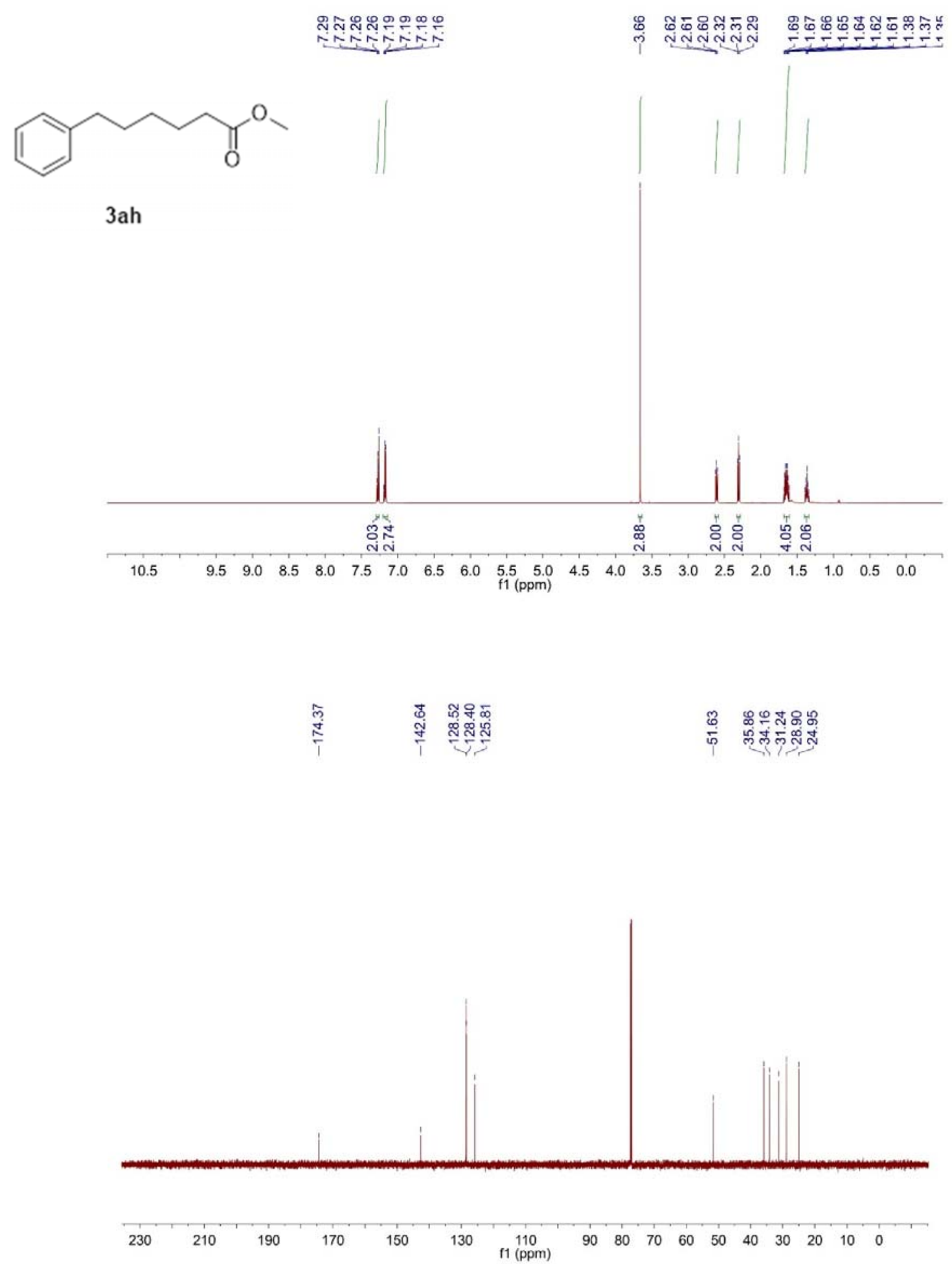

${ }^{1} \mathrm{H}$ NMR $(600 \mathrm{MHz})$ and ${ }^{13} \mathrm{C}\left\{{ }^{1} \mathrm{H}\right\}$ NMR $(151 \mathrm{MHz})$ spectra of 3ah (rt, $\left.\mathrm{CDCl}_{3}\right)$. 

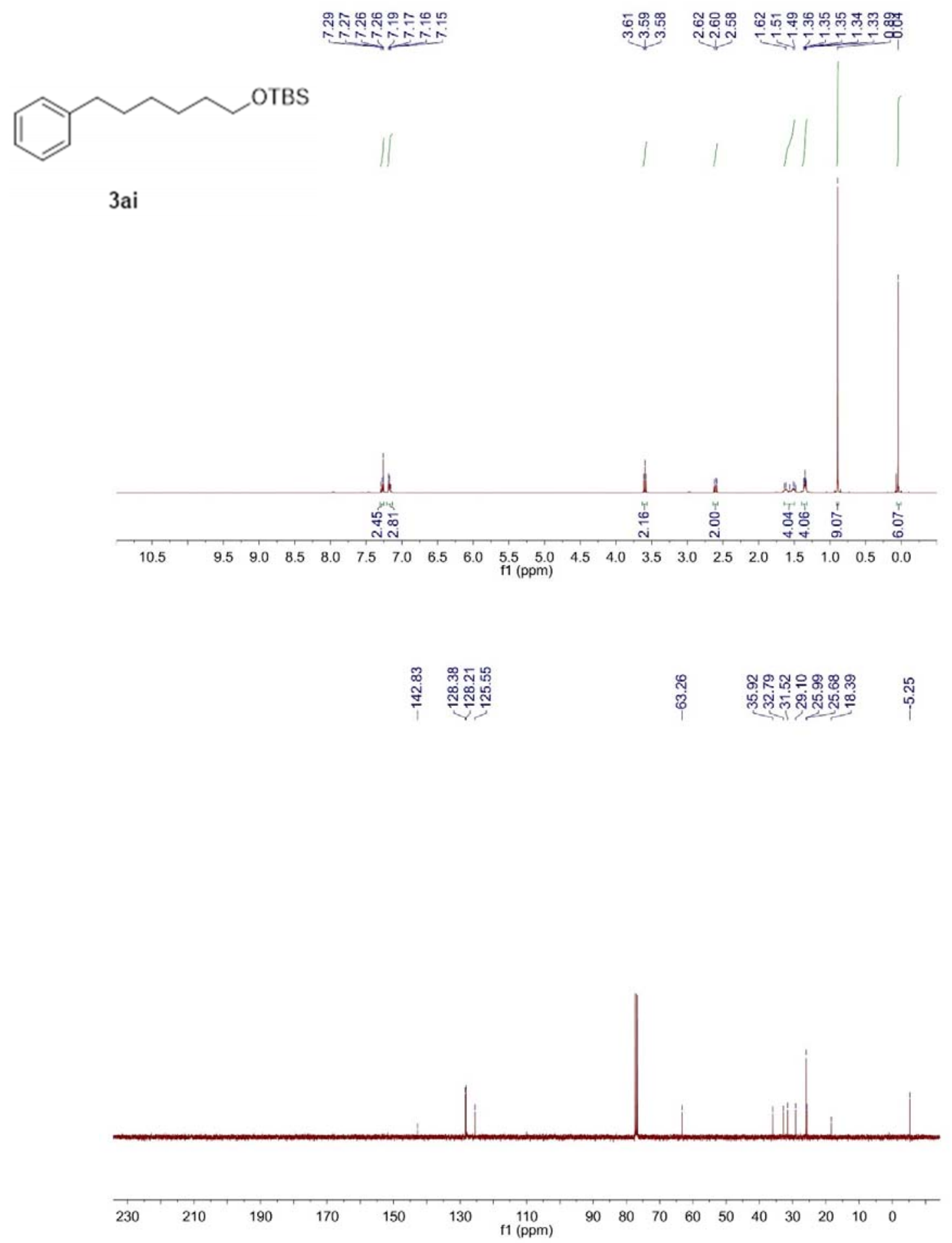

${ }^{1} \mathrm{H}$ NMR (400 MHz) and ${ }^{13} \mathrm{C}\left\{{ }^{1} \mathrm{H}\right\}$ NMR (101 MHz) spectra of 3ai $\left(\mathrm{rt}, \mathrm{CDCl}_{3}\right)$. 

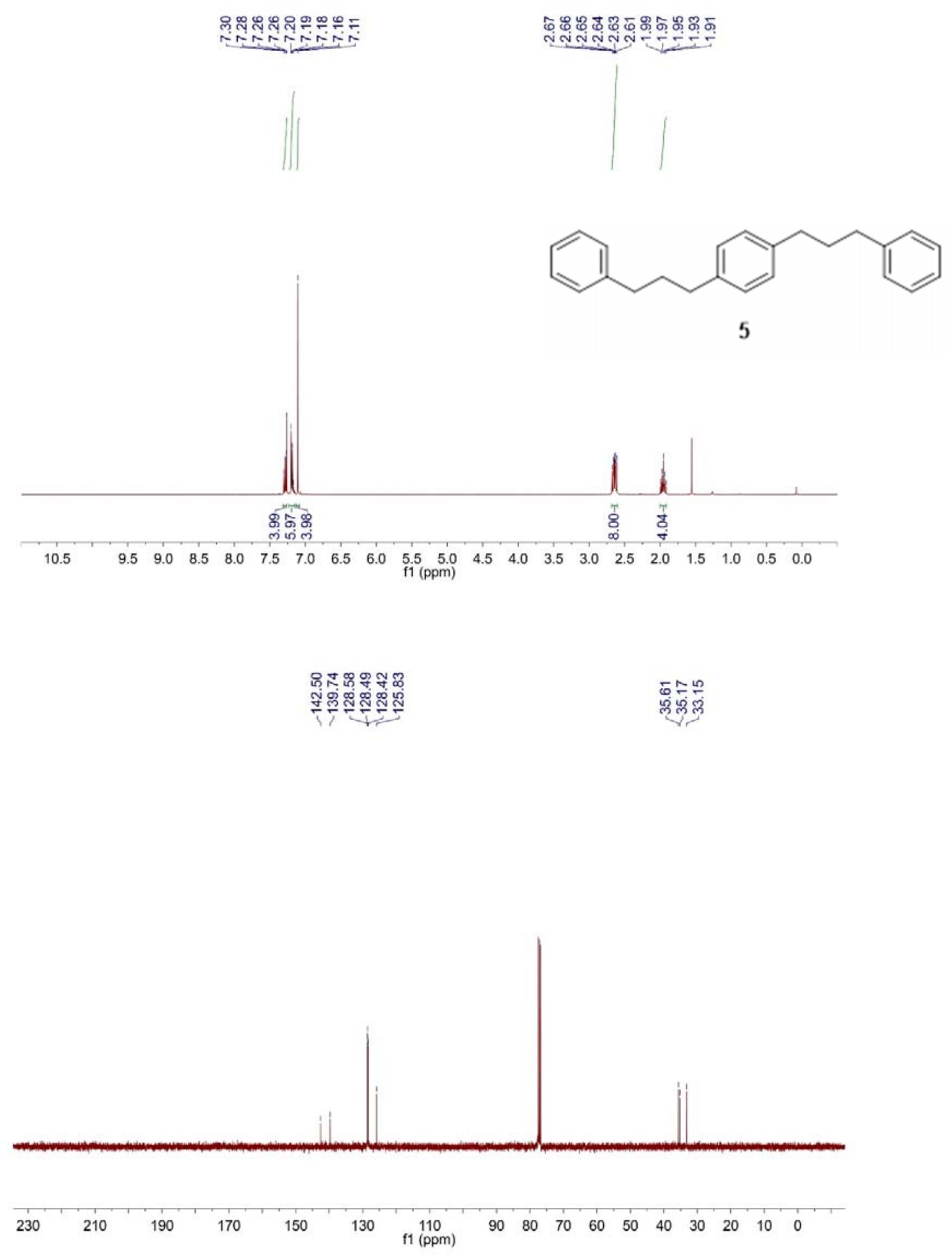

${ }^{1} \mathrm{H}$ NMR (400 MHz) and ${ }^{13} \mathrm{C}\left\{{ }^{1} \mathrm{H}\right\}$ NMR (101 MHz) spectra of $5\left(\mathrm{rt}, \mathrm{CDCl}_{3}\right)$. 


\section{References}

(1) (a) Okuda, Y.; Xu, J.; Ishida, T.; Wang, C.; Nishihara, Y. ACS Omega 2018, 3, 13129. (b) Wang, Z.; Wang, X.; Nishihara, Y. Chem. Commun. 2018, 54, 13969. (c) Wang, X.; Wang, Z.; Liu, L.; Asanuma, Y.; Nishihara, Y. Molecules 2019, 24, 1671. (d) Wang, X.; Wang, Z.; Asanuma, Y.; Nishihara, Y. Org. Lett. 2019, 21, 3640. (e) Wang, Z.; Wang, X.; Nishihara, Y. Catalysts 2019, 9, 574. (f) Wang, X.; Wang, Z.; Nishihara, Y. Chem. Commun. 2019, 55, 10507.

(2) Ding, S.; Xu, L.; Li, P. ACS Catal. 2016, 6, 1329.

(3) Crestoni, M. E. Chem. -Eur. J. 1998, 4, 993.

(4) Jin, W.; Wong, W.-T.; Law, G.-L. ChemCatChem 2014, 6, 1599.

(5) Moriya, T.; Takayama, K.; Konakahara, T.; Ogiwara, Y.; Sakai, N. Eur. J. Org. Chem. 2015, 2277.

(6) Matthias, C.; Kuck, D. Croat. Chem. Acta 2009, 82, 7.

(7) Khalaf, A. I.; Badman, C. E.; Ennis, M. P.; Horspool, W. M.; Sultana, Q. Trends in Photochemistry and Photobiology, 2010, 12, 1.

(8) Yamada, T.; Saito, K.; Akiyama, T. Adv. Synth. Catal. 2016, 358, 62.

(9) Amatore, M.; Gosmini, C. Chem. -Eur. J. 2010, 16, 5848.

(10) Cao, Q.; Howard, J. L.; Wheatley, E.; Browne, D. L. Angew. Chem. Int. Ed. 2018, 57, 11339.

(11) Ho, Y. A.; Leiendecker, M.; Liu, X.; Wang, C.; Alandini, N.; Rueping, M. Org. Lett. 2018, 20, 5644.

(12) Chatupheeraphat, A.; Liao, H.-H.; Srimontree, W.; Guo, L.; Minenkov, Y.; Poater, A.; Cavallo, L.; Rueping, M. J. Am. Chem. Soc. 2018, 140, 3724.

(13) Komiyama, T.; Minami, Y.; Hiyama, T. Chem. Lett. 2018, 47, 1048.

(14) Masson-Makdissi, J.; Vandavasi, J. K.; Newman, S. G. Org. Lett. 2018, 20, 4094.

(15) Prinsell, M. R.; Everson, D. A.; Weix, D. J. Chem. Commun. 2010, 46, 5743.

(16) Crockett, M. P.; Tyrol, C. C.; Wong, A. S.; Li, B.; Byers, J. A. Org. Lett. 2018, $20,5233$.

(17) Lee, J.-Y.; Fu, G. C. J. Am. Chem. Soc. 2003, 125, 5616.

(18) Xu, S.; Wu, G.; Ye, F.; Wang, X.; Li, H.; Zhao, X.; Zhang, Y.; Wang, J. Angew. Chem. Int. Ed. 2015, 54, 4669.

(19) Topley, A. C.; Isoni, V.; Logothetis, T. A.; Wynn, D.; Wadsworth, H.; Gibson, A. M. R.; Khan, I.; Wells, N. J.; Perrio, C.; Brown, R. C. D. Chem. -Eur. J. 2013, 19, 1720.

(20) Fürstner, A.; Martin, R.; Krause, H.; Seidel, G.; Goddard, R.; Lehmann, C. W. J. Am. Chem. Soc. 2008, 130,8773 .

(21) Laulhe, S.; Blackburn, J. M.; Roizen, J. L. Org. Lett. 2016, $18,4440$. 